\title{
How to Heat a Planet? Impact of Anthropogenic Landscapes on Earth's Albedo and Temperature
}

\author{
Mark Healey \\ Lindfield, Australia \\ Email: ma1healey@hotmail.com
}

How to cite this paper: Healey, M. (2020) How to Heat a Planet? Impact of Anthropogenic Landscapes on Earth's Albedo and Temperature. International Journal of Geosciences, 11, 420-457. https://doi.org/10.4236/ijg.2020.116022

Received: May 21, 2020

Accepted: June 27, 2020

Published: June 30, 2020

Copyright (อ 2020 by author(s) and Scientific Research Publishing Inc. This work is licensed under the Creative Commons Attribution International License (CC BY 4.0).

http://creativecommons.org/licenses/by/4.0/

\section{(c) (i) Open Access}

\begin{abstract}
Today anthropogenic climate change is underway and predicted future global temperatures vary significantly. However, the drivers of current climate change and their links to Earth's natural glacial cycle have yet to be fully resolved. Currently, many on a local level understand, and are exposed to, the heat energy generated by what's referred to as the urban heat island effect (UHI), whereby natural flora with higher albedos is replaced by manmade urban areas with lower albedos. This heat effect is not constrained to these regions and all anthropogenic surfaces with lower albedos need to be studied and quantified as the accumulated additional heat energy (infrared energy) is trapped within Earth's atmosphere and could affect the Earth on a planetary level. Deployed satellites have detected critical changes to Earth's albedo to lower levels, however the cause and impact of these changes have yet to be fully understood and incorporated into Global Circulation models (GCMs). Here it's shown that industrialization of anthropogenic landscape practices of the past century has displaced millions of square kilometres of naturally high albedo grasslands with lower albedo agricultural landscapes. Utilising a fundamental Energy Balance Model, (EBM) it's demonstrated these specific changes have generated vast amounts of additional heat energy which is trapped by the atmosphere, transferred and stored within the oceans of the Earth as shown in Figure 1. The total additional heat energy accumulated over the preceding 110 years correlates to that required to warm the Earth to the levels seen to date, altering Earth's overall energy budget. This energy will continue to accumulate and warm the Earth to a predicted $1.60 \pm 0.20$ Celsius by 2050 over 1910 levels. These findings are independent of anthropogenic Greenhouse Gas (GHG) additions and are further validated by predicting Earth's temperature and albedo at the last glacial maxima, suggesting that an albedo cycle aligned to Gaia theory is the primary driver of Earth's natural climate cycle.
\end{abstract}




\section{Keywords}

Earth Albedo, Anthropogenic Landscape Changes, Heat Fluxes, Earth's Energy Budget, Glacial Cycle, Gaia Links

\section{Introduction}

Anthropogenic landscapes have been around for millennia with mankind domesticating crops in abundant quantities to realize and exploit these new sustainable agricultural methods [1]. These practices have been overwhelmingly beneficial for humanity. Today, Earth's surface is vastly different to that of the early 1900's [2]. Currently anthropogenic landscapes are the largest disruptive development to the planet's ecosystem, with over $52 \pm 13$ million square kilometres (50\%) [3] of habitable land converted to agriculture/urban areas. This equates to $33 \%$ of the Earth's land surface. Many first think of deforestation as the major alteration when it comes to land clearing changes in the last century. However, it's been estimated up to $90 \%$ [4] of the Worlds Grasslands have suffered the greatest clearing and this has occurred at a faster rate than forests due to grassland's general topography, annual rainfall, rich dark fertile soils [5] and ease of conversion to cropland. As it stands, these areas are one of the least protected regions of the world [5]. The calculated conversion ratio between grassland to cropland and forest to cropland is estimated at (60\%:40\%) [2]. With the onset of industrialization, today, most grasslands have been converted into agricultural landscapes. The transformation of the World's natural landscapes to agricultural land has increased by $6.7 \pm 1.6$ million square kilometres within the last 110 years (Figure 2), and now estimated at $15.0 \pm 3.5$ million square kilometres (1/10 of the Earth's land area) [2]. The juxtapose heat flux or albedo properties of these altered flora surfaces has been overlooked in the causation climate change debate.

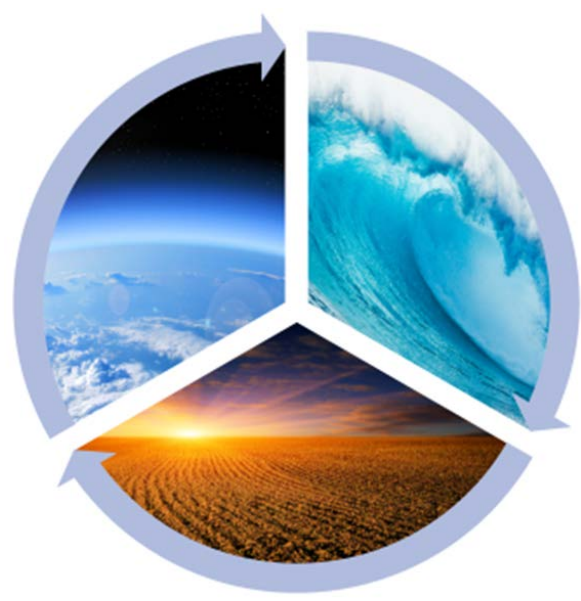

Figure 1. Mechanism for anthropogenic landscape heat energy production, trapped by the atmosphere and stored within the oceans. 


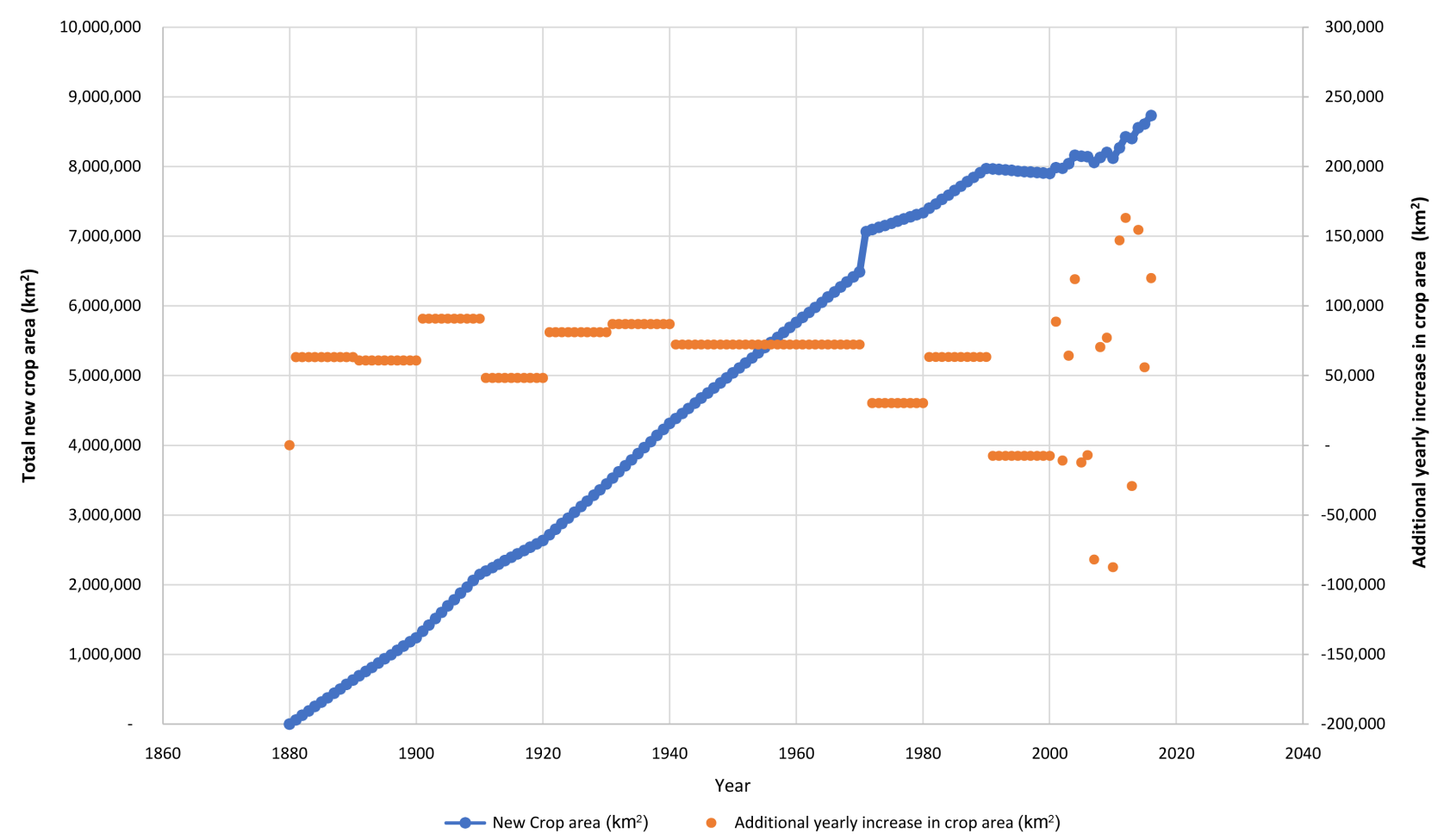

Figure 2. Total new global crop area (square kilometres) as well as additional yearly increase (square kilometres) on the secondary $\mathrm{y}$ axis from 1880-2016 [2].

While much attention has been focused on the correlated anthropogenic $\mathrm{CO}_{2}$, (Greenhouse Gas Emissions (GHG)) increases, these increases have lagged temperature rise and questions remain about the overall causation mechanism [6] and have marred the study of other climate drivers. Without fully accounting for anthropogenic landscape heat effects over the past 110 years, the conclusion drawn to date maybe casuistic. At present there is no alternate, credible theory to readily explain and account for the energy necessary to raise the global temperature by the levels seen. Furthermore, using the fundamental laws of thermodynamics encompassing energy conservation, additional accumulated heat energy generated from the removal \& conversion of $4.02 \pm 1.6$ million square kilometres of natural grasslands with high albedo properties to newly created anthropogenic darker agricultural landscapes with lower albedos, can be identified as the main contributor and causation of climate change [7].

The word Albedo (al-bee-doh) refers to a measure of how much light energy is reflected by a surface, or stays as short-wave energy, while the remaining amount is absorbed and transformed to longwave energy, i.e. heat/infrared radiation. Surfaces that appear whiter reflect most of the light energy that is radiated upon the surface and therefore has a high albedo, while darker surfaces absorbs/transforms most of the light energy into longwave, heat energy, indicating a lower albedo. The albedo scale is between 0 for full absorption to 1 for full reflection. This concept and its application are fundamentally important when dealing with climate change and anthropogenic alterations. 
While at first thought, it would appear inconceivable that such a simple change in albedo from grasslands to croplands would have the capability or potency to deliver the necessary energy to warm the entire planet within this time frame. However, a $10 \%-20 \%$ albedo reduction of this extremely large area, resulting in a net heat flux of (4.60 - 9.20 Watts per square meter), [8] can transform the sun's light energy to the heat energy required to increase the Earth's temperature, and lower the Earth's overserved terrestrial surface albedo and overall top of atmosphere (TOA) planetary albedo. While fully-grown crops such as wheat and corn albedos don't differ to that of the natural grasslands at certain growth phases and seasons of the year, the major variance is that at the start of the cropping cycle where bare cultivated soils with low albedos $0.11 \pm$ 0.01 [9] are fully exposed for extended periods of the season. This conditional state is dependent on rainfall/soil moisture for the crops to germinate and change the overall surface to higher albedo settings and associated lower heat fluxes. These areas are carefully ploughed, tilled, sprayed, limed and fertilized to ensure crop success. Furthermore, when dormant brown grasslands albedos of 0.36 [8] and a near-infrared reflectance of 0.58 at certain parts of the season are replaced by croplands with green growth phase albedos (visible leaf reflectance of 0.10 \& near-infrared reflectance of 0.58 ), ultimately changes the heat flux of this area and produces additional heat energy by up to $50 \%$ [8]. These changes lower the overall yearly average albedo of this area. The grasslands albedo buffering capabilities are removed and replaced with anthropogenic croplands incapable of displaying the exact albedo/heat flux properties within the yearly cropping cycle. This additional heat energy not only raises the temperature of the surrounding land surfaces, but this heat energy is contained, trapped and distributed within the atmosphere and oceans of the planet. The area of croplands has evolved and increased year on year since 1910 and is only now approaching peak agricultural/cropland area (Figure 2) [2]. To date, these changes have not been incorporated into GCMs as it has been assumed that such changes do not play a significant role in Earth's overall heat flux budget [10]. This measured change to heat fluxes and additional build-up of heat energy is correct for short durations, however over decades and centuries the accumulated energy has significant differences. The natural grasslands converted to grafted anthropogenic agricultural croplands have been judged to exhibit the exact same heat flux properties in all GCMs [11]. While deforestation and clearing of the world's forested areas with associated lower albedos $0.142 \pm 0.011$ to make way for agricultural land with higher albedos of $0.163 \pm 0.013$ have partially offset the temperature rise in this time frame [10], these areas are less favoured croplands as they are documented to be less fertile with lower productivity to that of grassland/cropland conversions [6]. These cooling changes and heat fluxes have been recognized and accounted for in the GCMs currently used [11].

It's been further recognized that, changes to surface albedos are powerful climate drivers of local, regional land areas and ultimately Earth's climate [12]. 
These changes are currently responsible for the Urban Heat Island effect (UHI) many already know far too well in our large sprawling cities of the World, sending temperatures to unprecedented high levels for extended periods [13]. This heat effect is not constrained to these man-made alterations and the additional heat energy produced from all anthropogenic landscapes with overall negative albedos, obey the laws of thermodynamics and flows to cooler regions, affecting the entire planet's energy balance, seasonal weather patterns and ultimately the Earths planetary albedo and temperature. This has been labelled here the Anthropogenic Landscape Heat Effect, (ALHE). The anthropogenic surfaces borne from the industrialized development of large parts of natural unaltered grasslands with higher albedos $(0.25 \pm 0.02)$ to that of cultivated croplands with lower albedos of $(0.20 \pm 0.02)$ or up to a $20 \%$ reduction, have not been given the necessary attention such a powerful heat energy source command. To give an example of the additional energy added to Earth's energy budget for every 0.01 albedo units lower than previous, every square kilometre produces an extra $2.94 \times$ $10^{13}$ Joules per year.

Moreover, concerns remain for the arctic region as the high albedo $(0.60 \pm .10)$ sea ice is slowly transformed to deep ocean low albedo $(0.08 \pm 0.02)$ properties due to the shorter winter season caused by the warmer ocean temperatures. Additionally, fires and burnt areas of the world are seemingly increasing in frequency and area and while these are not directly linked to causing global warming, they also contribute to lower albedo terrain. Both alterations will only further exacerbate the warming currently underway due to the positive feedbacks, however, have not been considered in the accumulated heat energy calculations performed herein.

In real terms additional worldwide cropland areas $(6.7 \pm 1.6$ million square kilometres) has roughly increased by $90 \%$ of the area of Australia (7.6 million square kilometres) within 110 years. The full impact of these anthropogenic landscape changes has only recently been introduced into some land surface models (LSM) and have been an area of consideration when it comes to the associated net energy flux impacts to the Earth's energy budget [4] [14]. While at the same time other research has touted increased albedo/reflectance changes with lower heat fluxes or new geo-engineering practices to cropland areas as a major way to fully mitigate the current global warming trend currently being experienced [15]. Ultimately, global warming is dictated by the iron-clad laws of thermodynamics. If the additional heat energy is more than the previous year the Earth warms. In reverse, the Earth cools and if the heat energy stays the same, the Earth's temperature remains unchanged.

\section{Methodology}

In the following calculations the Earth is considered using an Energy Balance Model (EBM). EBMs do not simulate the climate, but instead consider the balance between the energy entering the Earth's atmosphere from the sun and the heat released back out to space and are the foundation and basis upon which 
GCMs are built. The Earth is a unique planet in many ways. The existence of an atmosphere with greenhouse gases that's able to trap heat energy (infrared energy) is one such unique system, while allowing to pass shorter frequency energy back into space as the Earth radiates at a (TOA) black body temperature of 254 Kelvin ( -19 Celsius) with an albedo of 0.302 and current average observed temperature of 288 Kelvin ( 15 Celsius). A second is the existence of vast oceans filled with liquid saltwater. Saltwater has rare elementary properties that include a very high heat capacity of 3.89 Joules per gram per kelvin, which enables the oceans to act as a large heat sink that stabilizes the overall temperature of the planet. While the interaction between the atmosphere and oceans are very complex requiring super computers to run GCM models to predict future weather events, the overall energy balance is less so and basic EBMs, Budyko, Rakipova \& Sellers [16] [17] [18] utilized in the 1960's can be employed as a good fundamental starting point.

To heat the Planet, you must essentially heat the oceans that stores $93 \%$ of the energy [19]. Due to the immense size of the ocean, the epipelagic zone or top $150 \pm 50$ meters $\left(5.4 \times 10^{7} \pm 1.8 \times 10^{7}\right.$ cubic kilometres $)$ of the ocean can mix with the atmosphere, with deeper parts taking thousands of years to completely overturn and absorb additional energy and increase in temperature. Furthermore, it's been estimated that the oceans take 2640 years to fully overturn [20]. When considering the energy impacts of the past 110 years, this equates to $4 \%$ or $\left(5.4 \times 10^{7}\right.$ cubic kilometres) ocean volume. Eventually, the above-mentioned subtle observation and important interpretation of landscape heat flux changes, make vast differences to the Earth's energy budget and resulting land/ocean mean temperature.

Examining the NOAA global land \& ocean mean temperature chart from 1880 to present there is a clear inflection period in 1910-1911 whereby the overall 10 -year average temperature, post this year are higher than the previous year with some exceptions in the mid 1940 to the late 1970's. Today's temperature is 1.33 Celsius above 1910, or an additional 0.25 Celsius above the normally quoted 1880 temperature of 1.08 Celsius. Therefore, in this series of calculation, 1910 will be considered the starting year and zero temperature point. All modelled temperature simulations will be compared to this shortened adjusted NOAA 1910 zeroed temperature timeframe shown in Figure 3(i).

A second adjusted baseline temperature chart Figure 3(ii) consists of the standard NOAA global land \& ocean mean temperature chart and removes all the years having Atmospheric Atomic testing between 1946-1981, thus, shifting the 1981 temperature reading to 1946 . By excluding these years 1946-1981 due to the dawn of atmospheric atomic testing, this completely removes any atmospheric cooling effects that may have been experienced at this time period [21].

The modelled temperature simulations (1910-2050) charts can also be expressed as additional accumulated energy contained within the oceans/atmosphere, and NOAA currently measures this at $1.6 \times 10^{23}$ Joules above 1990 levels [22]. 


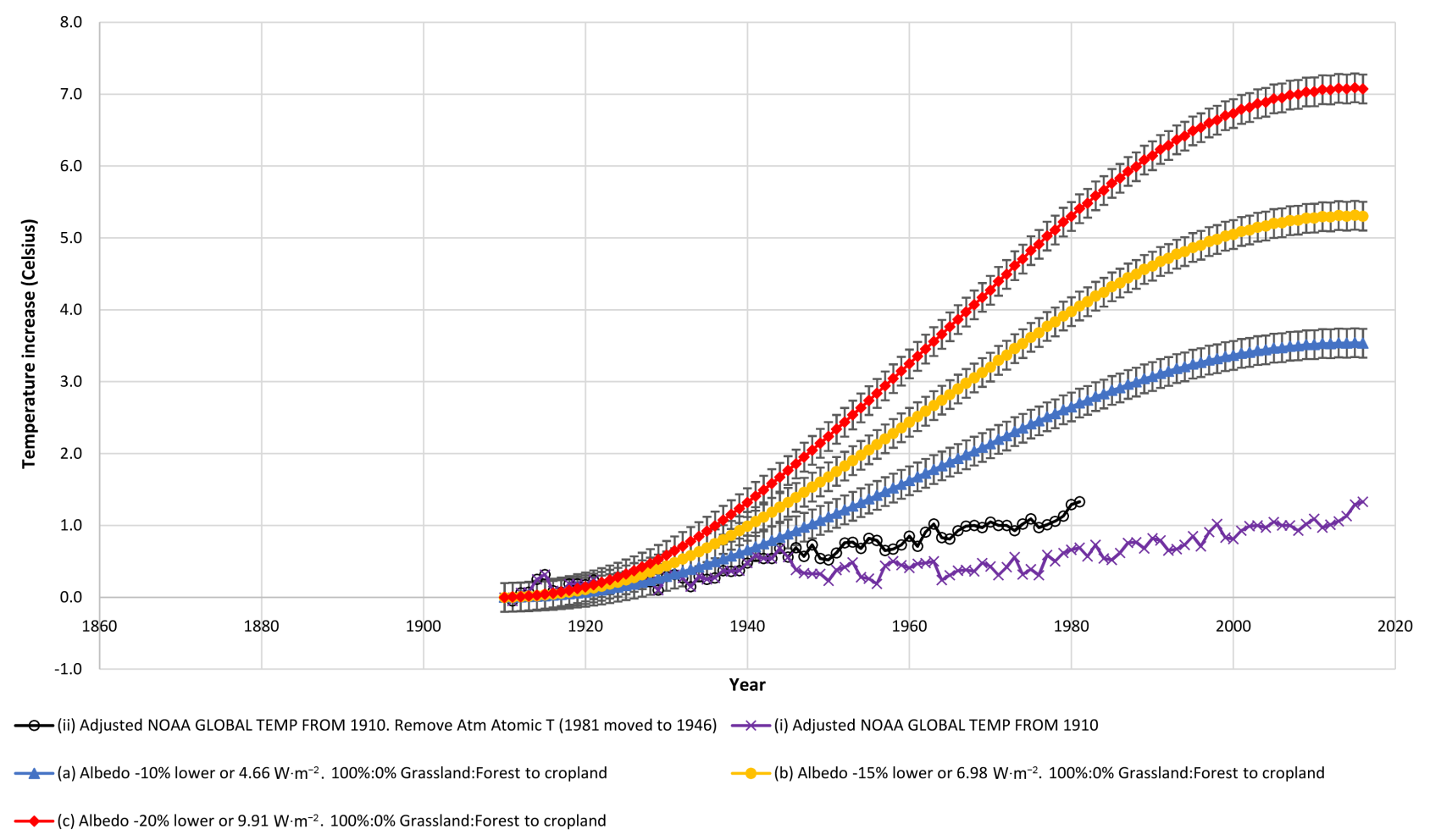

Figure 3. Global temperature increase (Celsius) chart (1910 zero temperature).

To start this series of temperature calculations, an average sea depth of $100 \mathrm{~m}$ or $3.6 \times 10^{19}$ Litres will be set. Utilizing the heat capacity of saltwater (3.89 Joules per gram per Kelvin or 3890 Joules per kilogram per Kelvin), this equates to 1.45 $\times 10^{23}$ Joules per Kelvin. Now applying the laws of thermodynamics, once the amount of accumulated additional heat energy reaches this figure the temperature of the Earth increases by 1-degree Kelvin or Celsius.

To continue the EBM calculations the following 7 assumptions are made:

1) The surface change for the averaged albedo difference of the converted land area (considering the cropping cycles) is constantly maintained year on year due to the same anthropogenic practices.

2) The area of the change is permanent, once the alteration has been initiated.

3) The amount of energy at the surface of the Earth is taken from the average surface energy of the planet obtained from Earth's Global Energy Budget figure, now estimated at 184.0 Watts per square meter [23].

4) Energy reflected by clouds and the Atmosphere is constantly maintained at 79 Watts per square meter [23], throughout this time period This may indeed overestimate the contribution of clouds in the early 1900's.

5) Average sunlight per square meter of terrain is 12 hours per day or $(43,200$ seconds per day), 365 days per year.

6) The amount of energy required to heat the Earth, 1 Celsius or Kelvin is estimated at $1.45 \times 10^{23}$ Joules based on $100 \mathrm{~m}$ ocean depth $\left(3.6 \times 10^{7}\right.$ cubic kilometres).

7) The remaining of Earth's radiation budget quantities and annual mean 
energy budget for the globe are taken from the Trenberth $E T A L$. work. As shown in Supplementary Table S1(a) and Table S1(b) [23], with 2002 as the benchmark year.

Formulae used to determine additional heat energy from anthropogenic landscape albedo alterations are based from the amount of heat $Q$ required to warm an object of mass $m$ by raising its temperature $\Delta T$ and is given by

$$
Q=m c \Delta T
$$

where $c$ is the specific heat of the material from which an object is made.

\section{Equation 1}

$$
\begin{aligned}
& \text { Yearly heat Energy(Joules) } \\
& =\text { Total changed area(square meters) } \\
& \times \text { Total daylight time(seconds) } \\
& \times \text { Difference in heat flux(Joules per second) } \\
& \text { - Previous Year Energy(Joules) }
\end{aligned}
$$

\section{Equation 2}

Total accumulated heat energy(Joules)(particular year)

$=$ Yearly heat Energy (Joules) + All previous yearly heat Energies (Joules)

\section{Equation 3}

Global temperature increase(Celsius)

$=$ Total accumulated heat energy $($ Joules $) / 1.45 \times 10^{23}$ Joules per Celsius

Earth's planetary albedo can be calculated utilizing the Stefan-Boltzmann law, with $T_{P}$ equal to average planetary temperature, with $S_{\text {ave }}$ equal to the top of atmosphere solar energy, and $a$ the Earth's albedo, $\sigma$ is the Stefan-Boltzmann constant.

Equation 4

$$
\begin{aligned}
& S_{\text {ave }}(1-\alpha)=\sigma T_{P}^{4} \\
& T_{P}=\left(S_{\text {ave }}(1-\alpha) / \sigma\right)^{1 / 4}
\end{aligned}
$$

\section{Results}

The first series of modelled temperature calculations derived from Equations (1)-(3) are performed using $10 \%, 15 \%$ and $20 \%$ reduction on the anthropogenic albedo surface alteration converting from grasslands to croplands for the entire introduced 6.7 million square kilometres anthropogenic area, with no accounting for the forest to cropland conversions resulting in a (100\%:0\%) ratio. The resulting additional heat flux is 4.60 Watts per square meter, 6.90 Watts per square meter and 9.20 Watts per square meter respectively.

All the temperature results overshot the expected baseline NOAA adjusted temperature and are shown in Figures 3(a)-(c).

A second series of temperature calculations are performed that accounts for forest to cropland conversions. Starting at a lowered 55\%:45\% (grassland to 
cropland):(forest to cropland) ratio. The resulting additional heat flux is 0.82 Watts per square meter, 2.10 Watts per square meter and 3.38 Watts per square meter respectively, totalling 6.7 million square kilometres shown in Figures 4(a)-(c). The resulting temperature chart 4(b) shows a Pearson correlation of 0.97 when compared to the adjusted NOAA temperature chart 4(ii), i.e., removing the atmospheric atomic testing years, and a paired $t$ test result of. $1.6 \times 10^{-10}$, making these highly probable they are correlated. The total energy (Joules) is plotted on the secondary y axis in Figure 4(e).

An additional temperature chart is constructed, shifting the resulting modelled temperatures from 1946 to 1981 thereby excluding the Atmospheric atomic testing years; resulting in Figures $5(\mathrm{a})-(\mathrm{c})$. These temperature charts can be used to predict the future global atmospheric/ocean mean temperatures and are compared to the 1910 adjusted NOAA temperature chart 5(i). To date, Earth's global temperature has risen 1.33 Celsius above 1910 levels, (an increase of 0.25 Celsius above the normally quoted 1880 temperature increase of 1.08 Celsius), here it's shown that additional accumulated heat energy generated from darker anthropogenic landscape changes totalling $2.28 \times 10^{23}$ Joules from 1910, will continue increasing Earth's temperature but not exceeding $1.60 \pm 0.20$ Celsius by 2050 as shown in Figure 5(b).

Utilising Equation (4), the Earth's TOA planetary albedo can be obtained. These changes have decreased the Earths, top of atmosphere (TOA) planetary albedo from 0.3160 in 1910 to 0.2987 in 2050 and are seen in Figure 6, which equates to 5.88 Watts per square meter, global energy flux difference.

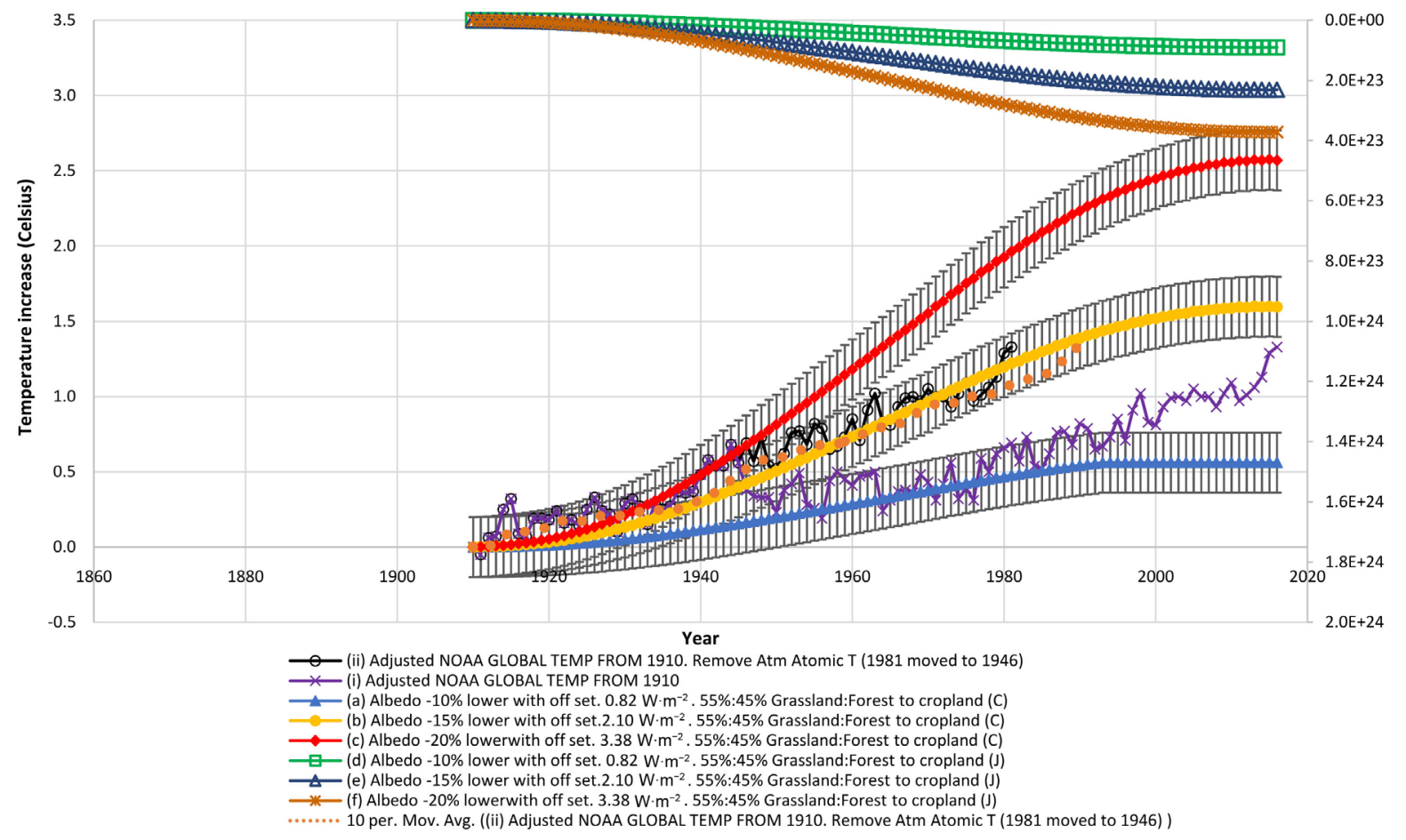

Figure 4. Global temperature increase (Celsius) chart, additional accumulated heat energy (Joules) plotted on the secondary y axis. 


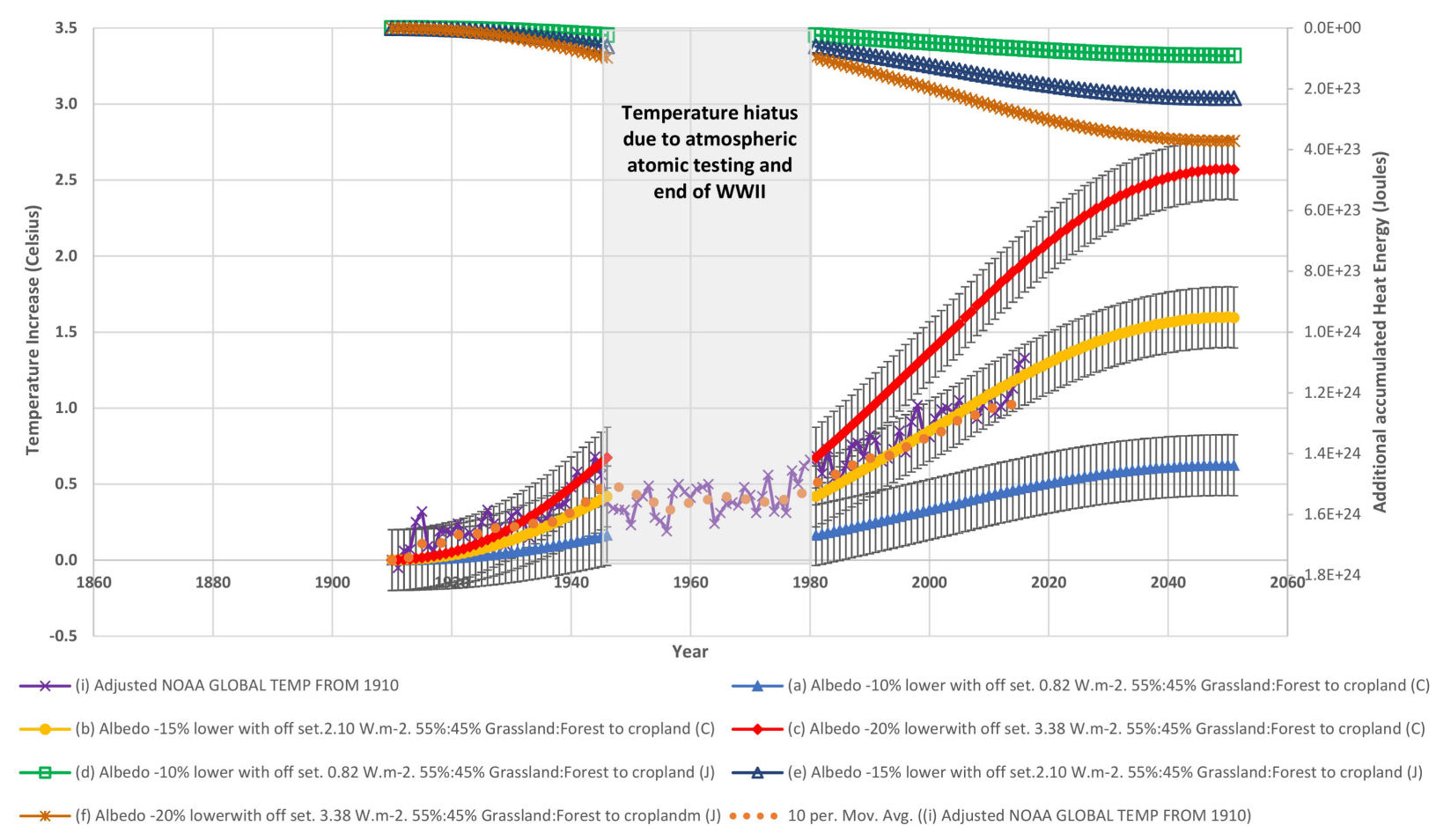

Figure 5. Global temperature increase (Celsius) chart, additional accumulated heat energy (Joules) plotted on the secondary (y) axis (1910 zero temperature). This chart includes the temperature hiatus due to atmospheric atomic testing and end of WWII.
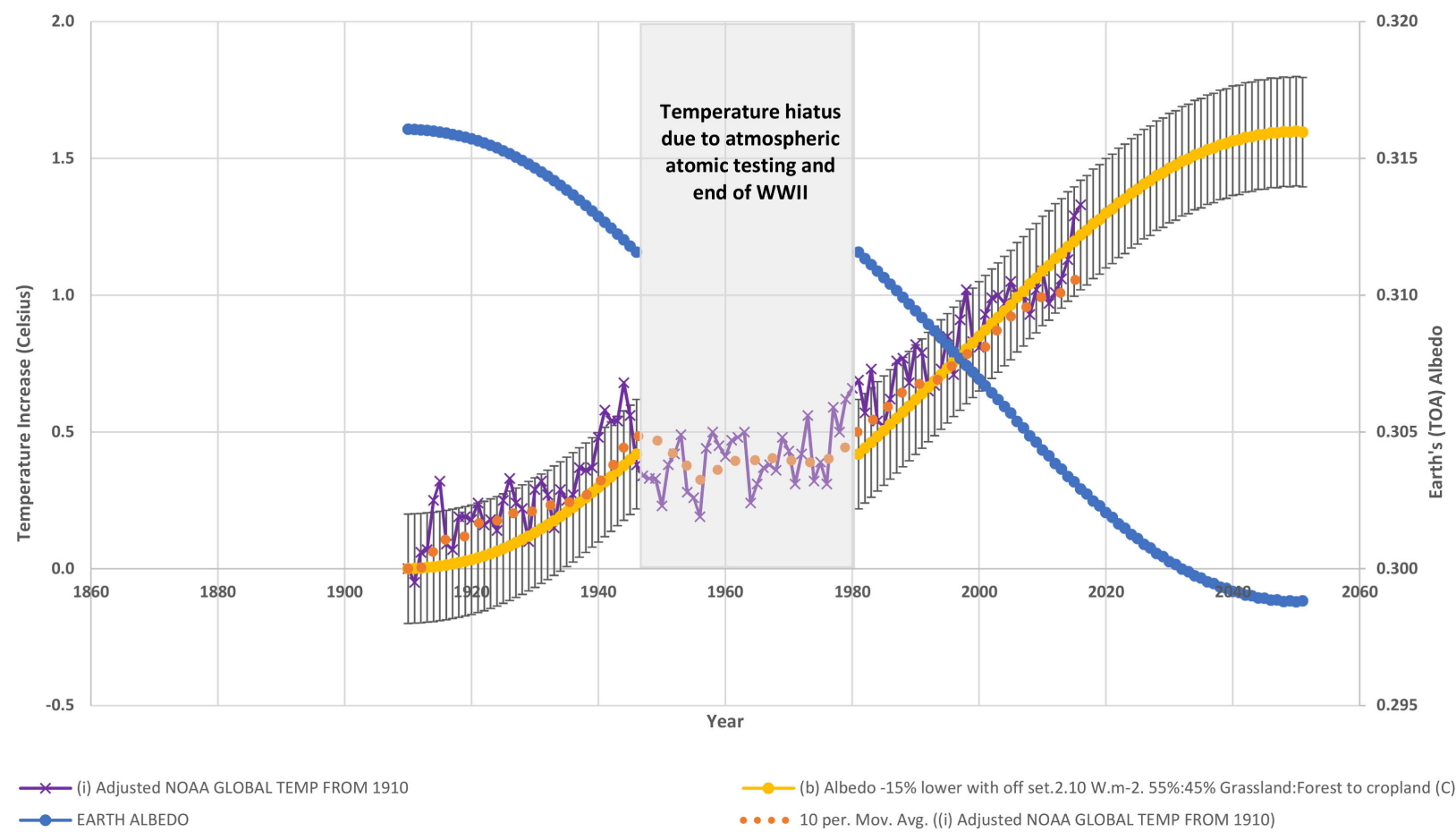

— (b) Albedo -15\% lower with off set.2.10 W.m-2. 55\%:45\% Grassland:Forest to cropland (C) ... 10 per. Mov. Avg. ((i) Adjusted NOAA GLOBAL TEMP FROM 1910)

Figure 6. Global temperature increase (Celsius) chart, with Earth's (TOA) albedo plotted on the secondary y axis (1910 zero temperature).

A further chart, Figure 7 is constructed to compare the predicted Earth's albedo and TOA temperature (Kelvin) again from the results of Equation (4). 


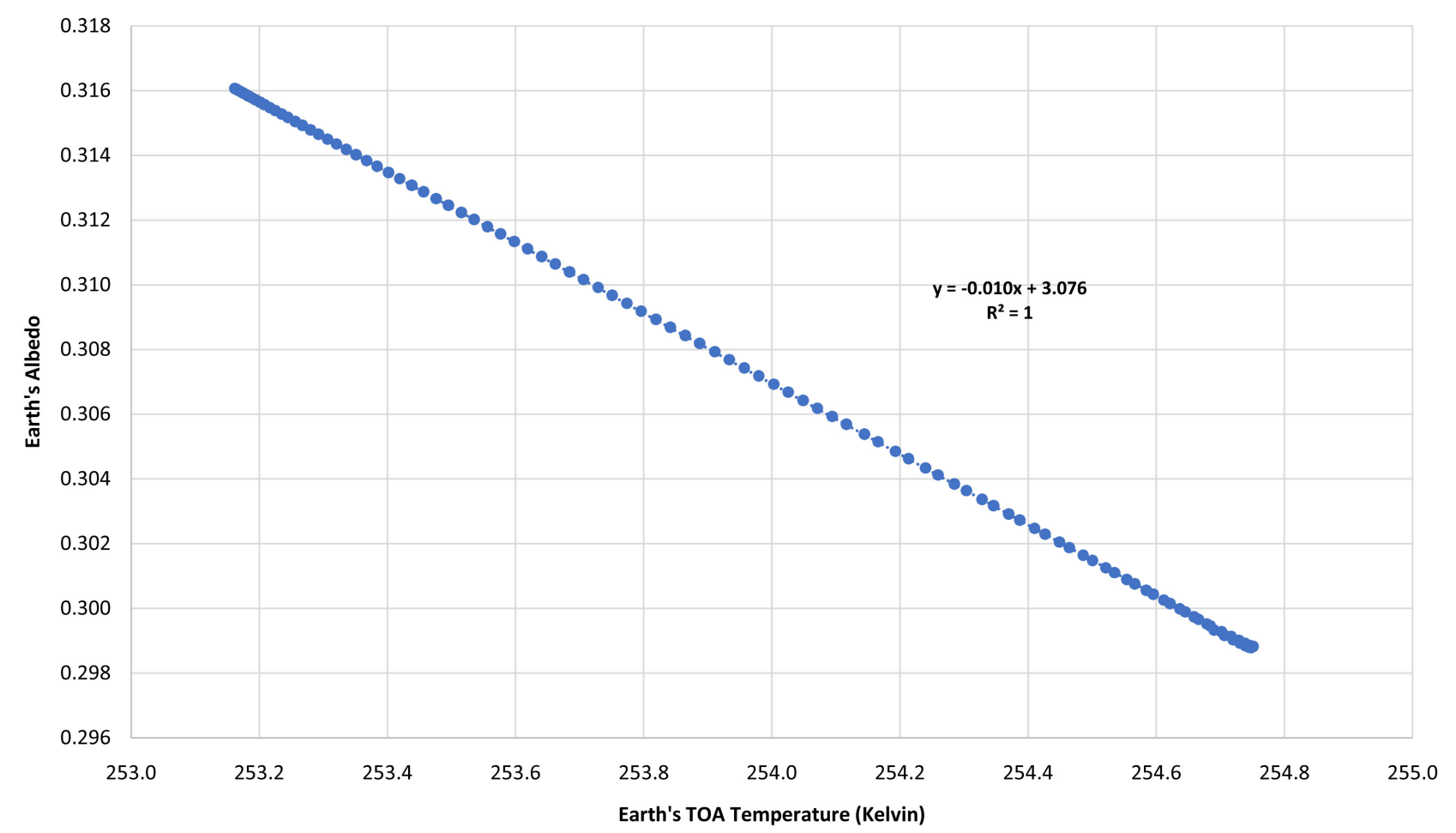

Figure 7. Earth's albedo versus Earth's TOA temperature (Kelvin).

This results in Equation (5) below:

$$
y=-0.0109 x+3.0769, R^{2}=1
$$

All calculations can be seen in supplementary spreadsheet (1) for calculations and summarized in Supplementary Table S2.

\section{Validation}

To validate this methodology and calculations, we subject them to the ultimate test by determining the Earth's temperature and albedo at the last glacial maxima some 20,000 years ago. This is the last known undisputed steady state of Earth, whereby $25 \%(36,760,564$ square kilometres) of the Earth's land surface was covered in glacial ice sheets [24] with a high surface albedo (0.6) replacing natural grasslands and forests at an estimated 60\%: 40\% ratio. This represents a newly calculated heat flux reduction for this area of $-73.21 \mathrm{~W}$ atts per square meter when compared to the previous $60 \%$ grassland: $40 \%$ forest surfaces ratio. Following that the entire oceans would completely overturn several times leading into the glacial maxima time frame, the total volume of ocean used in this calculation is $1.35 \times 10^{21}$ Litres. The total amount of heat energy entering into Earths system would therefore need to be substantially less to drive the Earth's observed temperature down to 281 Kelvin (8 Celsius) seen in the last glacial maxima some 7 Celsius below the current average global temperature 15 Celsius. This would equate to $-5.42 \times 10^{24}$ Joules per Celsius not entering the Earths system as heat energy (long wave infrared energy) and being reflected into space as short wave energy. 
These calculations driven by this immense area of high albedo ice area establishes the total energy decrease of $-4.49 \times 10^{25}$ Joules, equating to a $-8.29 \pm 0.20$ Kelvin or Celsius below current temperature or 246.4 Kelvin ( -26.6 Celsius) TOA and observed global temperature at 279.4 Kelvin (6.4 Celsius). Furthermore, the Earth's albedo can be calculated using the Stefan-Boltzmann Equation (4), which would increase to 0.385 at this time as seen in supplementary spreadsheet (8).

A revised glacial ice albedo of 0.55 for the entire glacial ice area ( -63.16 Watts per square meter) results in a calculated temperature of $-7.23 \pm 0.20$ Celsius below current temperature or 247.45 Kelvin TOA temperature and observed global temperature at 280.45 Kelvin. The Earth's albedo would be revised to 0.373 , well within the scope of the actual Earth's temperature recorded in the ice cores at this time.

To further understand and valid the methodology used, the Earth's albedo is calculated from Equation (5) obtained in the first series of calculations:

$$
y=-0.0109 x+3.0769, R^{2}=1
$$

The Earth's albedo is calculated for Last Glacial maxima temperature of 247.45 Kelvin, at 0.3784 , a very close result to the previous above 0.373 albedo obtained, or 1.84 Watts per square meter difference. All calculations can be seen in supplementary spreadsheet (1) for calculations and summarized in Supplementary Table S2.

\section{Discussion}

While there are some inherent errors that exist within the calculations performed, from the tessellated grafted anthropogenic surface area changes, associated land and cloud albedos and resulting heat fluxes that culminates in a \pm 0.2 Celsius estimated error, these are outweighed by the basic, fundamental laws of thermodynamics employed as well as the reasonable, logical assumptions made in the semiempirical calculations performed within the EBM. This combined with the validation calculations predicting Earth's temperature and albedo at the last glacial maxima, shows how large-scale albedo changes can drive the planetary temperatures experienced in Earth's past, and may indeed be aligned to an Earth albedo cycle associated with Gaia theory [25].

Looking closely at the recorded NOAA global temperatures from 1910 to present and comparing them to the modelled temperatures seen in Figure 6(b), the modelled results appear are on the lower side of the actual NOAA global temperatures, and only 10 of the 110 of the NOAA global temperatures yearly results fall outside the predicted error results. This stems from the conservative estimates for the anthropogenic induced heat fluxes and total converted areas utilized. While lower latent heat in the early 1900's may also contribute to additional heat energy entering the system due to reduced cloud formation and therefore less reflected energy resulting in higher transmission of energy reaching Earth's lowered albedo surfaces and ultimately transformed to additional 
heat energy. This would result in increased temperatures than otherwise modelled. Current research indicates the latent heat measurements have increased over the century, resulting in greater cloud formation and rainfall [26] [27]. However, from the results obtained by the two separate methods above, the heat fluxes used appear to be maintained and accurate, and within acceptable limits over the 110-year period.

\section{Conclusion}

In conclusion, this paper shows that utilising an EBM, the unintended additional accumulated heat energy as a consequence of all ALHE alterations, has year on year resulted in increased global average temperatures since 1910, except for the atmospheric atomic testing years (1946-81). A Pearson's correlation of 0.97 , as well as a paired $t$-test result of $1.6 \times 10^{-10}$ was calculated between these results and NOAA average global temperatures, indicating a strong correlation. Using the Stefan-Boltzmann law the Earth's average absorbed heat flux will increase by 5.88 Watts per square meter from 1910 to 2050, as an indirect result of Earth's TOA albedo decreasing from 0.3160 to 0.2987 over this timeframe. Without any contrary change, or off-setting, this additional accumulated heat energy totalling $2.29 \times 10^{23}$ Joules, will continue to enter Earth's system, warming the planet to a predicted temperature of $1.60 \pm 0.20$ Celsius above 1910 levels by 2050 and is independent of anthropogenic GHG increases. These findings should be closely studied and incorporated into more complex GCMs. If all global warming seen to date can be attributed to surface albedo changes, this gives rise to the question of whether surface albedo changes, may, in fact, have been a larger contributor to global temperature fluctuations seen in the distant past and therefore been the primary driver of natural climate change, i.e. Ice Ages/interglacial periods, and may indeed be aligned to an Earth albedo cycle associated with Gaia theory.

\section{Conflicts of Interest}

The author declares no conflicts of interest regarding the publication of this paper.

\section{References}

[1] Bar-Yosef, O. (1998) On the Nature of Transitions: The Middle to Upper Palaeolithic and the Neolithic Revolution. Cambridge Archaeological Journal, 8, 141. http://nrs.harvard.edu/urn-3:HUL.InstRepos:12211496 https://doi.org/10.1017/S0959774300001815

[2] Goldewijk, K. (2001) Estimating Global Land Use Change over the Past 300 Years. Global Biogeochemical Cycles, 15, 417-433. https://doi.org/10.1029/1999GB001232

[3] Goldewijk, K., Beusen, A., Doelman, J. and Stehfest, E. (2017) New Anthropogenic Land Use Estimates for the Holocene, Built-Up, Grazing \& Cropland; HYDE 3.2. http://themasites.pbl.nl/tridion/en/themasites/hyde

[4] Sterling, S. and Ducharne, A. (2008) Comprehensive Data Set of Global Land Cover Change for Land Surface Model Application. Global Biogeochemical Cycles, 22, GB3017. https://doi.org/10.1029/2007GB002959 
[5] Goriup, P. (1998) World Commission on Protected Areas (WCPA) of IUCN-The World Conservation Union, Newbury. Protected Areas Programme Parks, 8, 4.

[6] Humlum, O., Stordahl, K. and Solheim, J.E. (2013) The Phase Relation between Atmospheric Carbon Dioxide and Global Temperature. Global and Planetary Change, 100, 51. https://doi.org/10.1016/j.gloplacha.2012.08.008

[7] Pallé, E., Goode, P., Montañés-Rodríguez, P. and Koonin, S. (2004) Changes in Earth's Reflectance over the Past Two Decades. Science, 304, 1299-1301. https://doi.org/10.1126/science.1094070

[8] Twine, T., Kucharik, C. and Foley, A. (2004) Effects of Land Cover Change on the Energy and Water Balance of the Mississippi River Basin. Journal of Hydrometeorology, 5, 640-655. https://doi.org/10.1175/1525-7541(2004)005<0640:EOLCCO >2.0.CO;2

[9] Albedo, D.E. (2003) Encyclopedia of Soil Science.

[10] Findell, K.L., et al. (2007) Modelled Impact of anthropogenic Land Cover Change on Climate. Journal of Climate, 20, 3621-3634. https://doi.org/10.1175/JCLI4185.1

[11] De Oliveira Faria, T., Rodrigues, T., Curado, L., Gaio, D. and de Souza Nogueir, J. (2018) Surface Albedo in Different Land-Use and Cover Types in Amazon Forest Region. Revista Ambiente \& Água, 13, e2120.

https://doi.org/10.4136/ambi-agua.2120

[12] Sagan, C., Toon, O.B. and Pollack, J.B. (1979) Anthropogenic Albedo Changes and the Earth's Climate. Science New Series, 206, 1363-1368.

https://doi.org/10.1126/science.206.4425.1363

[13] Mohajerani, A., Bakaric, J. and Jeffrey-Bailey, T. (2017) The Urban Heat Island Effect, Its Causes, and Mitigation, with Reference to the Thermal Properties of Asphalt Concrete. Journal of Environmental Management, 197, 522-538. https://doi.org/10.1016/j.jenvman.2017.03.095

[14] Laguë, M.M., Bonan, G.B. and Swann, A.L.S. (2019) Separating the Impact of Individual Land Surface Properties on the Terrestrial Surface Energy Budget in both the Coupled and Uncoupled Land-Atmosphere System. Journal of Climate, 32, 5725-5744. https://doi.org/10.1175/JCLI-D-18-0812.1

[15] Davin, E., Seveviratne, S.I., et al. (2014) Preferential Cooling of Hot Extremes Form Cropland Albedo Management. Proceedings of the National Academy of Sciences of the United States of America, 111, 9757-9761. https://doi.org/10.1073/pnas.1317323111

[16] Budyko, M.I. (1966) Polar Ice \& Climate. In: Arctic Heat Budget and Atmospheric Circulation, Memo RM-5233-NSF, The RAND Corporation, Santa Monica, 3-21.

[17] Rakipova, S. (1966) The Influence of Arctic Ice Cover on the Zonal Distribution of Atmospheric Temperature. In: Arctic Heat Budget and Atmospheric Circulation, Memo. RM-5233-NSF, The RAND Corporation, Santa Monica, 411-441.

[18] Sellers, W. (1969) A Global Climatic Model Based on the Energy Balance of the Earth-Atmosphere System. Journal of Applied Meteorology, 8, 392-400. https://doi.org/10.1175/1520-0450(1969)008<0392:AGCMBO>2.0.CO;2

[19] Dahlman, L. and Lindsey (2018) Climate Change. Ocean Heat Content. NOAA Climate.gov.

[20] Henshaw, P.C., Charlson, R.J. and Burges, S.J. (2000) Water and the Hydrosphere, 6.2.2 Turnover Times. International Geophysics, 72, 109-131. https://doi.org/10.1016/S0074-6142(00)80112-6

[21] Robock, A., et al. (2007) Climatic Consequences of Regional Nuclear Conflicts. At- 
mospheric Chemistry and Physics, 7, 2003-2012.

https://doi.org/10.5194/acp-7-2003-2007

[22] Levitus, S., et al. (2012) World Ocean Heat Content and Thermosteric Sea Level Change (0-2000 m) 1955-2010. Geophysical Research Letters, 39, L10603.

https://doi.org/10.1029/2012GL051106

[23] Trenberth, K., Fasullo, J.T. and Kiehl, J. (2009) Earth's Global Energy Budget. American Meteorological Society, Boston. https://doi.org/10.1175/2008BAMS2634.1

[24] USGS-Glacier and Landscape Change in Response to Changing Climate Glaciers and Sea Level.

https://www2.usgs.gov/climate_landuse/glaciers/glaciers_sea_level.asp

[25] Lovelock, J. (2009) The Vanishing Face of Gaia. Basic Books, New York, 255.

[26] Kirkby, J., Duplissy, J., Sengupta, K., et al. (2016) Ion-Induced Nucleation of Pure Biogenic Particles. Nature, 533, 521-526. https://doi.org/10.1038/nature17953

[27] Singh, A.K. and Bhargawa, A. (2020) Delineation of Possible Influence of Solar Variability and Galactic Cosmic Rays on Terrestrial Climate Parameters. Advances in Space Research, 65, 1831-1842. https://doi.org/10.1016/j.asr.2020.01.006 


\section{Supplementary}

Table S1. (a) Top of atmosphere (TOA) mean radiation budget quantities clouds and the Earth's radiation energy system from 1910 to 2050. Global. The downward solar (Solar in), reflected solar (Solar reflected), and net (NET down) radiation are given with the ASR and OLR $\left(\mathrm{W} \cdot \mathrm{m}^{-2}\right)$, and albedo is given in percentage. The values start with figures from Earth's Global energy budget. Trenberth al et American Meterological society March 2009 [23]; (b) Top of atmosphere (TOA) mean radiation budget quantities clouds and the Earth's radiation energy system from 1910 to 2050. Global. The downward solar (Solar in), reflected solar (Solar reflected), and net (NET down) radiation are given with the ASR and OLR $\left(\mathrm{W} \cdot \mathrm{m}^{-2}\right)$, and albedo is given in percentage. The values start with figures from Earth's Global energy budget. Trenberth et al. American Meteorological society March 2009 [23]. Removed atmospheric atomic testing years, i.e. the 1946 temperature readings are and restart at 1981 . The bench mark year is highlighted as 2002.

(a)

\begin{tabular}{|c|c|c|c|c|c|c|c|c|c|c|c|c|c|c|c|c|c|}
\hline 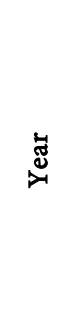 & 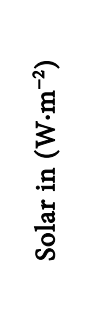 & 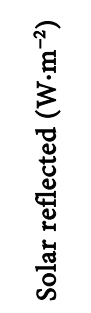 & 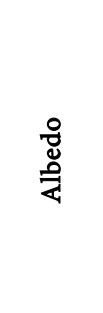 & 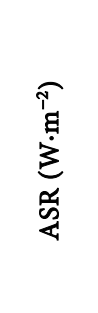 & 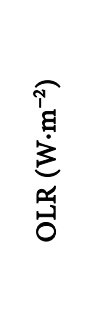 & 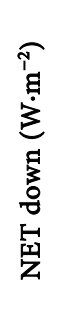 & 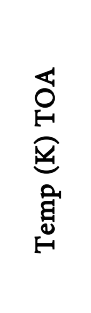 & 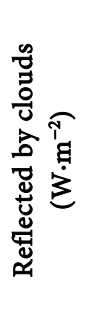 & 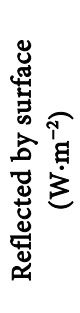 & 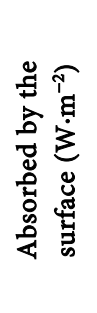 & 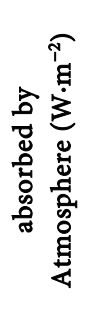 & 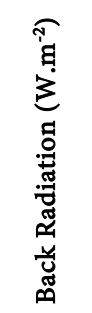 & 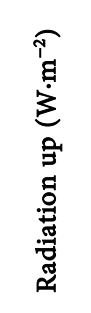 & 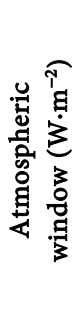 & 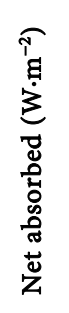 & 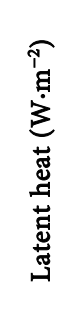 & 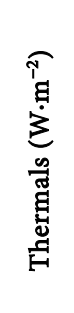 \\
\hline 1910 & 341.70 & 107.66 & $31.51 \%$ & 234.04 & 233.14 & 0.90 & 253.16 & 79.00 & 28.66 & 155.08 & 78.00 & 333.00 & 392.71 & 40.00 & 0.90 & 80.00 & 17.00 \\
\hline 1911 & 341.70 & 107.66 & $31.51 \%$ & 234.04 & 233.14 & 0.90 & 253.16 & 79.00 & 28.66 & 155.08 & 78.00 & 333.00 & 392.71 & 40.00 & 0.90 & 80.00 & 17.00 \\
\hline 1912 & 341.70 & 107.65 & $31.50 \%$ & 234.05 & 233.15 & 0.90 & 253.16 & 79.00 & 28.65 & 155.09 & 78.00 & 333.00 & 392.72 & 40.00 & 0.90 & 80.00 & 17.00 \\
\hline 1913 & 341.70 & 107.64 & $31.50 \%$ & 234.06 & 233.16 & 0.90 & 253.17 & 79.00 & 28.64 & 155.09 & 78.00 & 333.00 & 392.73 & 40.00 & 0.90 & 80.00 & 17.00 \\
\hline 1914 & 341.70 & 107.64 & $31.50 \%$ & 234.06 & 233.16 & 0.90 & 253.17 & 79.00 & 28.64 & 155.10 & 78.00 & 333.00 & 392.73 & 40.00 & 0.90 & 80.00 & 17.00 \\
\hline 1915 & 341.70 & 107.62 & $31.50 \%$ & 234.08 & 233.18 & 0.90 & 253.17 & 79.00 & 28.62 & 155.12 & 78.00 & 333.00 & 392.75 & 40.00 & 0.90 & 80.00 & 17.00 \\
\hline 1916 & 341.70 & 107.61 & $31.49 \%$ & 234.09 & 233.19 & 0.90 & 253.18 & 79.00 & 28.61 & 155.13 & 78.00 & 333.00 & 392.76 & 40.00 & 0.90 & 80.00 & 17.00 \\
\hline 1917 & 341.70 & 107.60 & $31.49 \%$ & 234.10 & 233.20 & 0.90 & 253.18 & 79.00 & 28.60 & 155.14 & 78.00 & 333.00 & 392.77 & 40.00 & 0.90 & 80.00 & 17.00 \\
\hline 1918 & 341.70 & 107.58 & $31.48 \%$ & 234.12 & 233.22 & 0.90 & 253.18 & 79.00 & 28.58 & 155.16 & 78.00 & 333.00 & 392.79 & 40.00 & 0.90 & 80.00 & 17.00 \\
\hline 1919 & 341.70 & 107.56 & $31.48 \%$ & 234.14 & 233.24 & 0.90 & 253.19 & 79.00 & 28.56 & 155.18 & 78.00 & 333.00 & 392.81 & 40.00 & 0.90 & 80.00 & 17.00 \\
\hline 1920 & 341.70 & 107.54 & $31.47 \%$ & 234.16 & 233.26 & 0.90 & 253.19 & 79.00 & 28.54 & 155.20 & 78.00 & 333.00 & 392.83 & 40.00 & 0.90 & 80.00 & 17.00 \\
\hline 1921 & 341.70 & 107.52 & $31.46 \%$ & 234.18 & 233.28 & 0.90 & 253.20 & 79.00 & 28.52 & 155.22 & 78.00 & 333.00 & 392.85 & 40.00 & 0.90 & 80.00 & 17.00 \\
\hline 1922 & 341.70 & 107.49 & $31.46 \%$ & 234.21 & 233.31 & 0.90 & 253.21 & 79.00 & 28.49 & 155.25 & 78.00 & 333.00 & 392.88 & 40.00 & 0.90 & 80.00 & 17.00 \\
\hline 1923 & 341.70 & 107.46 & $31.45 \%$ & 234.24 & 233.34 & 0.90 & 253.22 & 79.00 & 28.46 & 155.28 & 78.00 & 333.00 & 392.91 & 40.00 & 0.90 & 80.00 & 17.00 \\
\hline 1924 & 341.70 & 107.43 & $31.44 \%$ & 234.27 & 233.37 & 0.90 & 253.22 & 79.00 & 28.43 & 155.31 & 78.00 & 333.00 & 392.94 & 40.00 & 0.90 & 80.00 & 17.00 \\
\hline 1925 & 341.70 & 107.39 & $31.43 \%$ & 234.31 & 233.41 & 0.90 & 253.23 & 79.00 & 28.39 & 155.35 & 78.00 & 333.00 & 392.98 & 40.00 & 0.90 & 80.00 & 17.00 \\
\hline 1926 & 341.70 & 107.36 & $31.42 \%$ & 234.34 & 233.44 & 0.90 & 253.24 & 79.00 & 28.36 & 155.38 & 78.00 & 333.00 & 393.01 & 40.00 & 0.90 & 80.00 & 17.00 \\
\hline 1927 & 341.70 & 107.31 & $31.41 \%$ & 234.39 & 233.49 & 0.90 & 253.26 & 79.00 & 28.31 & 155.43 & 78.00 & 333.00 & 393.06 & 40.00 & 0.90 & 80.00 & 17.00 \\
\hline 1928 & 341.70 & 107.27 & $31.39 \%$ & 234.43 & 233.53 & 0.90 & 253.27 & 79.00 & 28.27 & 155.47 & 78.00 & 333.00 & 393.10 & 40.00 & 0.90 & 80.00 & 17.00 \\
\hline 1929 & 341.70 & 107.22 & $31.38 \%$ & 234.48 & 233.58 & 0.90 & 253.28 & 79.00 & 28.22 & 155.51 & 78.00 & 333.00 & 393.15 & 40.00 & 0.90 & 80.00 & 17.00 \\
\hline 1930 & 341.70 & 107.18 & $31.37 \%$ & 234.52 & 233.62 & 0.90 & 253.29 & 79.00 & 28.18 & 155.56 & 78.00 & 333.00 & 393.19 & 40.00 & 0.90 & 80.00 & 17.00 \\
\hline 1931 & 341.70 & 107.13 & $31.35 \%$ & 234.57 & 233.67 & 0.90 & 253.31 & 79.00 & 28.13 & 155.61 & 78.00 & 333.00 & 393.24 & 40.00 & 0.90 & 80.00 & 17.00 \\
\hline 1932 & 341.70 & 107.07 & $31.34 \%$ & 234.63 & 233.73 & 0.90 & 253.32 & 79.00 & 28.07 & 155.66 & 78.00 & 333.00 & 393.29 & 40.00 & 0.90 & 80.00 & 17.00 \\
\hline 1933 & 341.70 & 107.02 & $31.32 \%$ & 234.68 & 233.78 & 0.90 & 253.34 & 79.00 & 28.02 & 55.72 & 78.00 & 333.00 & 393.35 & 40.00 & 0.90 & 80.00 & 17.00 \\
\hline
\end{tabular}




\section{Continued}

\begin{tabular}{|c|c|c|c|c|c|c|c|c|c|c|c|c|c|c|c|c|c|}
\hline 934 & 41.70 & 6.96 & $1.30 \%$ & 34.74 & 33.84 & 0.90 & 253.35 & 79.00 & 27.96 & 155.78 & 78.00 & 333.00 & 393.41 & 40.00 & 0.90 & 80.00 & 17. \\
\hline 35 & 41.70 & 6.90 & $.28 \%$ & 4.80 & 33.90 & .90 & 253.37 & 9.00 & 27.90 & 155.84 & 78.00 & 333.00 & 393.47 & 40.00 & 0.90 & 80.00 & 17. \\
\hline 36 & 41.70 & 6.84 & $.27 \%$ & 4.86 & 3.96 & .90 & 253.38 & 9.00 & 27.84 & 155. & 8.00 & 33.00 & 393.53 & 40.00 & 0.90 & 30.00 & 17. \\
\hline 37 & 1.70 & 6.77 & $25 \%$ & .93 & .03 & 90 & 253.40 & 9.00 & 27.77 & 55.97 & 8.00 & 3.00 & 393.60 & 40.00 & .90 & 0.00 & 17. \\
\hline 38 & 41.70 & 6.71 & $.23 \%$ & 4.99 & 34.09 & .90 & 253.42 & 79.00 & 27.71 & 156.03 & 78.00 & 333.00 & 393.66 & 40.00 & 0.90 & 80.00 & 17.0 \\
\hline 39 & 41.70 & 6.64 & $.21 \%$ & 5.06 & 34.16 & 0.90 & 253.44 & 9.00 & 27.64 & 156.10 & 78.00 & 333.00 & 393.73 & 40.00 & 0.90 & 30.00 & 17. \\
\hline 940 & 1.70 & 6.57 & $.19 \%$ & 5.13 & 234.23 & 0.90 & 253.46 & 9.00 & 27.57 & 156.17 & 8.00 & 333.00 & 393.80 & 40.00 & 0.90 & 0.00 & 17. \\
\hline 941 & 11.70 & 6.50 & $.17 \%$ & 5.20 & 34.30 & .90 & 253.48 & 9.00 & 27.50 & 156.24 & 78.00 & 333.00 & 393.87 & 40.00 & 0.90 & 0.00 & 17.0 \\
\hline 42 & 1.70 & 6.43 & $.15 \%$ & 5.27 & 34.37 & .90 & 253.50 & 9.00 & 27.43 & 156.31 & 8.00 & 33.00 & 393.94 & 40.00 & 0.90 & 0.00 & 7.0 \\
\hline 43 & 11.70 & 6.35 & $.12 \%$ & 5.35 & 34.45 & .90 & 253.52 & 79.00 & 27.35 & 156.39 & 78.00 & 333.00 & 394.02 & 40.00 & 0.90 & 30.00 & 17.0 \\
\hline 944 & 41.70 & 6.28 & $.10 \%$ & 5.42 & 34.52 & .90 & 253.54 & 9.00 & 27.28 & 156.46 & 8.00 & 333.00 & 394.09 & 40.00 & 0.90 & 0.00 & 17. \\
\hline 945 & 1.70 & 6.13 & $.06 \%$ & 5.57 & 34.67 & .90 & 253.58 & 9.00 & 7.13 & 156. & 78.00 & 3.00 & 394 & 40.00 & 0.90 & 0.00 & 17.0 \\
\hline 946 & 1.70 & 6.13 & $.06 \%$ & 5.57 & 34.67 & .90 & 253.58 & 9.00 & 27.1264 & 156.6126 & 0000 & 333.00 & 394. & 0.00 & & 0.00 & 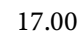 \\
\hline 47 & 1.70 & 6.05 & $.04 \%$ & 5.65 & 34.75 & 90 & 253.60 & 9.00 & .0468 & 156.6922 & 8.0000 & 3.00 & 3 & 0.00 & 00 & 0.00 & \\
\hline 948 & 11.70 & 5.97 & $.01 \%$ & 5.73 & 34.83 & .90 & 253.62 & 9.00 & 26.9694 & 156.7696 & 78.0000 & 333.00 & 394.40 & 40.00 & .9000 & 80.00 & 17. \\
\hline 49 & 1.70 & 5.89 & $.99 \%$ & 5.81 & 34.91 & .90 & 253.64 & 9.00 & 5.8880 & 156.8510 & 78.0000 & 33.00 & 394.48 & 40.00 & 9000 & 80.00 & 17. \\
\hline 50 & 1.70 & 5.81 & $.97 \%$ & 5.89 & 99 & .90 & 253.66 & 9.00 & 39 & 301 & 000 & & 394. & 0.00 & & 0.00 & $7 .($ \\
\hline 51 & 1.70 & 5.73 & $.94 \%$ & 5.97 & & & 253.68 & 9.00 & 9 & & 000 & 3.00 & 394 & 0.00 & & 0.00 & \\
\hline 52 & 1.70 & 5.65 & $.92 \%$ & 6.05 & 15 & & 253.71 & 9.00 & 5.6454 & 7.0936 & 78.0000 & 3.00 & 94. & 00 & 00 & 0.00 & \\
\hline 953 & 41.70 & 5.56 & $.89 \%$ & 6.14 & 35.24 & .90 & 253.73 & 9.00 & 26.5610 & 157.1780 & 78.0000 & 33.00 & 394.81 & 40.00 & 0.9000 & 80.00 & 17. \\
\hline 54 & 41.70 & 5.48 & $.87 \%$ & 6.22 & 35.32 & .90 & 253.75 & 9.00 & 6.4793 & 157.2597 & 78.0000 & 3.00 & 394.89 & 40.00 & 0.9000 & 80.00 & 17. \\
\hline 55 & 1.70 & & $.84 \%$ & & & & & 9.00 & & & & & & & & & \\
\hline 56 & 1.70 & 5.31 & $.82 \%$ & 6.39 & & & 53.80 & 9.00 & 5.31 & 4279 & 000 & .00 & 395 & 4 & 0 & 0.00 & \\
\hline 57 & 1.70 & 5.22 & $.79 \%$ & 6.48 & 58 & .90 & 253.82 & 9.00 & 6.2248 & 57.5142 & 0000 & 3.00 & 395.14 & 0 & 00 & 0.00 & \\
\hline 58 & 41.70 & 5.14 & $.77 \%$ & 6.56 & 35.66 & & 253.84 & 9.00 & 26.1414 & 157.5976 & 78.0000 & 3.00 & 395.23 & 40.00 & 0 & 80.00 & 17. \\
\hline 59 & 41.70 & & & & & & & & & & & & & & & 30.00 & \\
\hline 960 & 1.70 & 1.97 & $.72 \%$ & 6.73 & & & & 9.00 & & 7.7684 & & & & & & & \\
\hline 961 & 1.70 & 4.88 & $.69 \%$ & 6.82 & & & 253.91 & 9.00 & & & 000 & & 395 & & 00 & .00 & \\
\hline 962 & 1.70 & 4.80 & $.67 \%$ & 6.90 & 00 & 90 & 253.93 & 9.00 & & .9398 & 000 & 3.00 & 395.57 & & & .00 & \\
\hline 963 & 41.70 & 4.71 & & & & & & 9.00 & & & & & & & & 0.00 & \\
\hline 964 & 1.70 & 4.63 & $62 \%$ & 7.07 & & & & 9.00 & 5.6277 & & 78.0000 & & & & .9000 & 0.00 & \\
\hline 965 & 1.70 & 4.54 & $.59 \%$ & 7.16 & & & 254.00 & 79.00 & & 1986 & 0000 & & & & 9000 & 0.00 & \\
\hline 966 & 1.70 & 1.46 & $\%$ & 7.24 & & & 2 & 9.00 & 5 & & 0000 & 3.00 & & & 9000 & 0.00 & \\
\hline 967 & 41.70 & 4.37 & $.54 \%$ & 7.33 & & & 254.05 & 79.00 & 25.3696 & 158.3694 & 78.0000 & 33.00 & 396.00 & 40.00 & 0.9000 & 80.00 & 17. \\
\hline 968 & 1.70 & 4.29 & & & & & 25 & & & & & & & & & & \\
\hline 969 & 41.70 & 4.20 & $.49 \%$ & 7.50 & 6.60 & 0.90 & 254.09 & 79.00 & 25.2000 & 8.5390 & 78.0000 & 3.00 & 396.17 & 40.00 & 0.9000 & 80.00 & . \\
\hline 7( & 1.70 & 4.12 & $.47 \%$ & 7.58 & 236.68 & 0.90 & 254.12 & 79.00 & 25.1174 & 58.6217 & 78.0000 & 333.00 & 396.25 & 40.00 & 0.9000 & 80.00 & \\
\hline
\end{tabular}




\section{Continued}

\begin{tabular}{|c|c|c|c|c|c|c|c|c|c|c|c|c|c|c|c|c|c|}
\hline 1971 & 341.70 & 104.01 & $30.44 \%$ & 237.69 & 236.79 & 0.90 & 254.14 & 79.00 & 25.0127 & 158.7263 & 78.0000 & 333.00 & 396.36 & 40.00 & 0.9000 & 80.00 & 17.00 \\
\hline 1972 & 341.70 & 103.93 & $30.42 \%$ & 237.77 & 236.87 & 0.90 & 254.17 & 79.00 & 24.9331 & 158.8060 & 78.0000 & 333.00 & 396.44 & 40.00 & 0.9000 & 80.00 & 17.00 \\
\hline 1973 & 341.70 & 103.83 & $30.39 \%$ & 237.87 & 236.97 & 0.90 & 254.19 & 79.00 & 24.8314 & 158.9076 & 78.0000 & 333.00 & 396.54 & 40.00 & 0.9000 & 80.00 & 17.00 \\
\hline 974 & 341.70 & 103.75 & $30.36 \%$ & 237.95 & 237.05 & 0.90 & 254.21 & 79.00 & 24.7549 & 158.9841 & 78.0000 & 333.00 & 396.61 & 40.00 & 0.9000 & 80.00 & 17.00 \\
\hline 975 & 341.70 & 103.66 & $30.34 \%$ & 238.04 & 237.14 & 0.90 & 254.24 & 79.00 & 24.6564 & 159.0826 & 78.0000 & 333.00 & 396.71 & 40.00 & 0.9000 & 80.00 & 17.00 \\
\hline 1976 & 341.70 & 103.58 & $30.31 \%$ & 238.12 & 237.22 & 0.90 & 254.26 & 79.00 & 24.5831 & 159.1560 & 78.0000 & 333.00 & 396.79 & 40.00 & 0.9000 & 80.00 & 17.00 \\
\hline 1977 & 341.70 & 103.49 & $30.29 \%$ & 238.21 & 237.31 & 0.90 & 254.28 & 79.00 & 24.4878 & 159.2512 & 78.0000 & 333.00 & 396.88 & 40.00 & 0.9000 & 80.00 & 17.00 \\
\hline 1978 & 341.70 & 103.42 & $30.27 \%$ & 238.28 & 237.38 & 0.90 & 254.30 & 79.00 & 24.4178 & 159.3212 & 78.0000 & 333.00 & 396.95 & 40.00 & 0.9000 & 80.00 & 17.00 \\
\hline 979 & 341.70 & 103.33 & $30.24 \%$ & 238.37 & 237.47 & 0.90 & 254.33 & 79.00 & 24.3259 & 159.4131 & 78.0000 & 333.00 & 397.04 & 40.00 & 0.9000 & 80.00 & 17.00 \\
\hline 1980 & 341.70 & 103.26 & $30.22 \%$ & 238.44 & 237.54 & 0.90 & 254.35 & 79.00 & 24.2593 & 159.4798 & 78.0000 & 333.00 & 397.11 & 40.00 & 0.9000 & 80.00 & 17.00 \\
\hline 1981 & 341.70 & 103.17 & $30.19 \%$ & 238.53 & 237.63 & 0.90 & 254.37 & 79.00 & 24.1698 & 159.5692 & 78.0000 & 333.00 & 397.20 & 40.00 & 0.9000 & 80.00 & 17.00 \\
\hline 1982 & 341.70 & 103.11 & $30.17 \%$ & 238.59 & 237.69 & 0.90 & 254.39 & 79.00 & 24.1058 & 159.6332 & 78.0000 & 333.00 & 397.26 & 40.00 & 0.9000 & 80.00 & 17.00 \\
\hline 1983 & 341.70 & 103.02 & $30.15 \%$ & 238.68 & 237.78 & 0.90 & 254.41 & 79.00 & 24.0191 & 159.7199 & 78.0000 & 333.00 & 397.35 & 40.00 & 0.9000 & 80.00 & 17.00 \\
\hline 984 & 341.70 & 102.96 & $30.13 \%$ & 238.74 & 237.84 & 0.90 & 254.43 & 79.00 & 23.9579 & 159.7812 & 78.0000 & 333.00 & 397.41 & 40.00 & 0.9000 & 80.00 & 17.00 \\
\hline 985 & 341.70 & 102.87 & $30.11 \%$ & 238.83 & 237.93 & 0.90 & 254.45 & 79.00 & 23.8740 & 159.8650 & 78.0000 & 333.00 & 397.50 & 40.00 & 0.9000 & 80.00 & 17.00 \\
\hline 1986 & 341.70 & 102.82 & $30.09 \%$ & 238.88 & 237.98 & 0.90 & 254.46 & 79.00 & 23.8159 & 159.9232 & 78.0000 & 333.00 & 397.55 & 40.00 & 0.9000 & 80.00 & 17.00 \\
\hline 1987 & 341.70 & 102.74 & $30.07 \%$ & 238.96 & 238.06 & 0.90 & 254.49 & 79.00 & 23.7352 & 160.0038 & 78.0000 & 333.00 & 397.63 & 40.00 & 0.9000 & 80.00 & 17.00 \\
\hline 1988 & 341.70 & 102.68 & $30.05 \%$ & 239.02 & 238.12 & 0.90 & 254.50 & 79.00 & 23.6802 & 160.0588 & 78.0000 & 333.00 & 397.69 & 40.00 & 0.9000 & 80.00 & 17.00 \\
\hline 989 & 341.70 & 102.60 & $30.03 \%$ & 239.10 & 238.20 & 0.90 & 254.52 & 79.00 & 23.6029 & 160.1361 & 78.0000 & 333.00 & 397.77 & 40.00 & 0.9000 & 80.00 & 17.00 \\
\hline 1990 & 341.70 & 102.55 & $30.01 \%$ & 239.15 & 238.25 & 0.90 & 254.53 & 79.00 & 23.5515 & 160.1876 & 78.0000 & 333.00 & 397.82 & 40.00 & 0.9000 & 80.00 & 17.00 \\
\hline 1991 & 341.70 & 102.48 & $29.99 \%$ & 239.22 & 238.32 & 0.90 & 254.55 & 79.00 & 23.4792 & 160.2598 & 78.0000 & 333.00 & 397.89 & 40.00 & 0.9000 & 80.00 & 17.00 \\
\hline 1992 & 341.70 & 102.43 & $29.98 \%$ & 239.27 & 238.37 & 0.90 & 254.57 & 79.00 & 23.4328 & 160.3062 & 78.0000 & 333.00 & 397.94 & 40.00 & 0.9000 & 80.00 & 17.00 \\
\hline 1993 & 341.70 & 102.37 & $29.96 \%$ & 239.33 & 238.43 & 0.90 & 254.58 & 79.00 & 23.3656 & 160.3734 & 78.0000 & 333.00 & 398.00 & 40.00 & 0.9000 & 80.00 & 17.00 \\
\hline 994 & 341.70 & 102.32 & $29.95 \%$ & 239.38 & 238.48 & 0.90 & 254.60 & 79.00 & 23.3243 & 160.4147 & 78.0000 & 333.00 & 398.05 & 40.00 & 0.9000 & 80.00 & 17.00 \\
\hline 1995 & 341.70 & 102.26 & $29.93 \%$ & 239.44 & 238.54 & 0.90 & 254.61 & 79.00 & 23.2622 & 160.4768 & 78.0000 & 333.00 & 398.11 & 40.00 & 0.9000 & 80.00 & 17.00 \\
\hline 1996 & 341.70 & 102.23 & $29.92 \%$ & 239.47 & 238.57 & 0.90 & 254.62 & 79.00 & 23.2259 & 160.5131 & 78.0000 & 333.00 & 398.14 & 40.00 & 0.9000 & 80.00 & 17.00 \\
\hline 997 & 341.70 & 102.17 & $29.90 \%$ & 239.53 & 238.63 & 0.90 & 254.64 & 79.00 & 23.1688 & 160.5702 & 78.0000 & 333.00 & 398.20 & 40.00 & 0.9000 & 80.00 & 17.00 \\
\hline 1998 & 341.70 & 102.14 & $29.89 \%$ & 239.56 & 238.66 & 0.90 & 254.65 & 79.00 & 23.1375 & 160.6015 & 78.0000 & 333.00 & 398.23 & 40.00 & 0.9000 & 80.00 & 17.00 \\
\hline 999 & 341.70 & 102.09 & $29.88 \%$ & 239.61 & 238.71 & 0.90 & 254.66 & 79.00 & 23.0854 & 160.6536 & 78.0000 & 333.00 & 398.28 & 40.00 & 0.9000 & 80.00 & 17.00 \\
\hline 2000 & 341.70 & 102.06 & $29.87 \%$ & 239.64 & 238.74 & 0.90 & 254.67 & 79.00 & 23.0592 & 160.6798 & 78.0000 & 333.00 & 398.31 & 40.00 & 0.9000 & 80.00 & 17.00 \\
\hline 2001 & 341.70 & 102.01 & $29.85 \%$ & 239.69 & 238.79 & 0.90 & 254.68 & 79.00 & 23.0108 & 160.7282 & 78.0000 & 333.00 & 398.36 & 40.00 & 0.9000 & 80.00 & 17.00 \\
\hline 2002 & 341.70 & 101.99 & $29.85 \%$ & 239.71 & 238.81 & 0.90 & 254.68 & 79.00 & 22.9896 & 160.7494 & 78.0000 & 333.00 & 398.38 & 40.00 & 0.9000 & 80.00 & 17.00 \\
\hline 2003 & 341.70 & 101.97 & $29.84 \%$ & 239.73 & 238.83 & 0.90 & 254.69 & 79.00 & 22.9685 & 160.7705 & 78.0000 & 333.00 & 398.40 & 40.00 & 0.9000 & 80.00 & 17.00 \\
\hline 2004 & 341.70 & 101.92 & $29.83 \%$ & 239.78 & 238.88 & 0.90 & 254.70 & 79.00 & 22.9244 & 160.8146 & 78.0000 & 333.00 & 398.45 & 40.00 & 0.9000 & 80.00 & 17.00 \\
\hline 2005 & 341.70 & 101.91 & $29.82 \%$ & 239.79 & 238.89 & 0.90 & 254.71 & 79.00 & 22.9070 & 160.8320 & 78.0000 & 333.00 & 398.46 & 40.00 & 0.9000 & 80.00 & 17.00 \\
\hline 2006 & 341.70 & 101.87 & $29.81 \%$ & 239.83 & 238.93 & 0.90 & 254.72 & 79.00 & 22.8681 & 160.8709 & 78.0000 & 333.00 & 398.50 & 40.00 & 0.9000 & 80.00 & 17.00 \\
\hline 07 & 341.70 & 101.86 & $29.81 \%$ & 239.84 & 238.94 & 0.90 & 254.72 & 79.00 & 22.8559 & 160.8831 & 78.0000 & 333.00 & 398.51 & 40.00 & 0.9000 & 80.00 & 17.00 \\
\hline
\end{tabular}




\section{Continued}

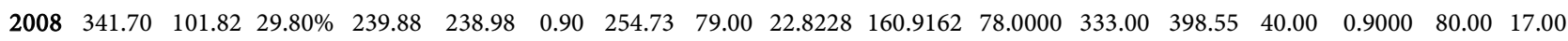

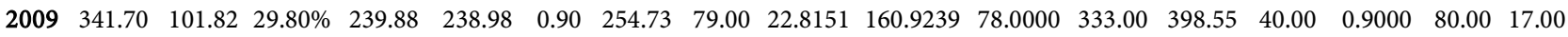

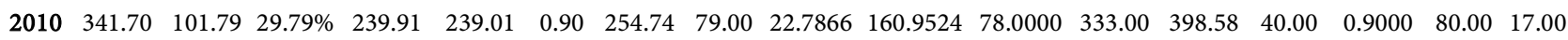

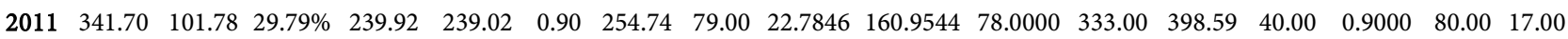

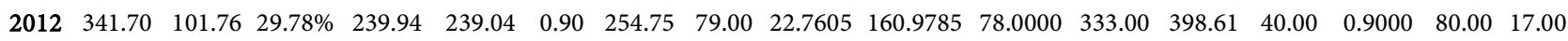

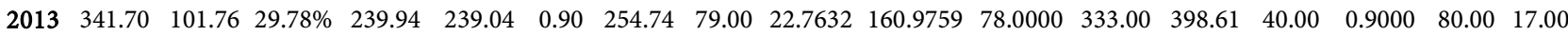

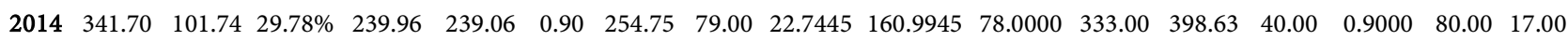

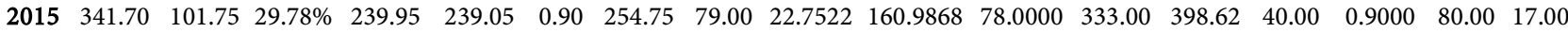

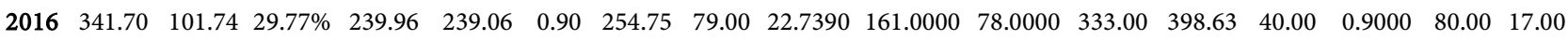

$2017 \quad 341.7$

$2018 \quad 341.7$

$2019 \quad 341.7$

$2020 \quad 341.7$

2021341.7

$2022 \quad 341.7$

$2023 \quad 341.7$

$2024 \quad 341.7$

$2025 \quad 341.7$

$2026 \quad 341.7$

$2027 \quad 341.7$

$2028 \quad 341.7$

$2029 \quad 341.7$

$2030 \quad 341.7$

2031341.7

$2032 \quad 341.7$

$2033 \quad 341.7$

$2034 \quad 341.7$

$2035 \quad 341.7$

$2036 \quad 341.7$

$2037 \quad 341.7$

$2038 \quad 341.7$

$2039 \quad 341.7$

$2040 \quad 341.7$

2041341.7

$2042 \quad 341.7$

$2043 \quad 341.7$

$2044 \quad 341.7$

$2045 \quad 341.7$

$2046 \quad 341.7$

$2047 \quad 341.7$

$2048 \quad 341.7$

2049341.7

$2050 \quad 341.7$

$2051 \quad 341.7$ 
(b)

\begin{tabular}{|c|c|c|c|c|c|c|c|c|c|c|c|c|c|c|c|c|c|}
\hline 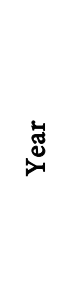 & 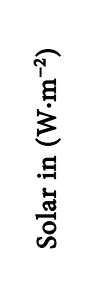 & 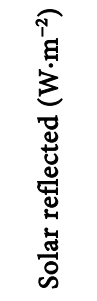 & 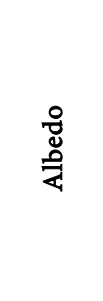 & 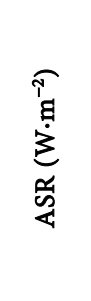 & 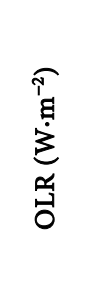 & 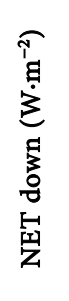 & 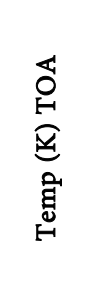 & 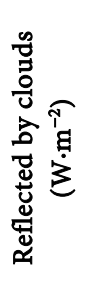 & 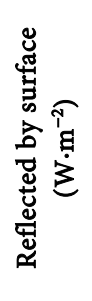 & 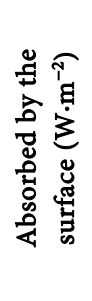 & 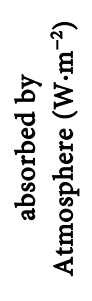 & 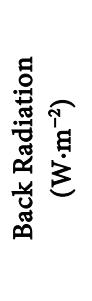 & 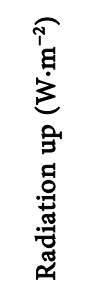 & 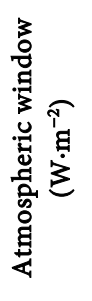 & 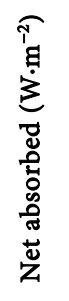 & 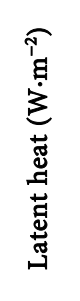 & 苗 \\
\hline 1910 & 341.70 & 107.66 & $31.51 \%$ & 234.04 & 233.14 & 0.90 & 253.16 & 79.00 & 28.66 & 155.08 & 78.00 & 333.00 & 392.71 & 40.00 & 0.90 & 80.00 & 17.00 \\
\hline 1911 & 341.70 & 107.66 & $31.51 \%$ & 234.04 & 233.14 & 0.90 & 253.16 & 79.00 & 28.66 & 155.08 & 78.00 & 333.00 & 392.71 & 40.00 & 0.90 & 80.00 & 17.00 \\
\hline 1912 & 341.70 & 107.65 & $31.50 \%$ & 234.05 & 233.15 & 0.90 & 253.16 & 79.00 & 28.65 & 155.09 & 78.00 & 333.00 & 392.72 & 40.00 & 0.90 & 80.00 & 17.00 \\
\hline 1913 & 341.70 & 107.64 & $31.50 \%$ & 234.06 & 233.16 & 0.90 & 253.17 & 79.00 & 28.64 & 155.09 & 78.00 & 333.00 & 392.73 & 40.00 & 0.90 & 80.00 & 17.00 \\
\hline 1914 & 341.70 & 107.64 & $31.50 \%$ & 234.06 & 233.16 & 0.90 & 253.17 & 79.00 & 28.64 & 155.10 & 78.00 & 333.00 & 392.73 & 40.00 & 0.90 & 80.00 & 17.00 \\
\hline 1915 & 341.70 & 107.62 & $31.50 \%$ & 234.08 & 233.18 & 0.90 & 253.17 & 79.00 & 28.62 & 155.12 & 78.00 & 333.00 & 392.75 & 40.00 & 0.90 & 80.00 & 17.00 \\
\hline 1916 & 341.70 & 107.61 & $31.49 \%$ & 234.09 & 233.19 & 0.90 & 253.18 & 79.00 & 28.61 & 155.13 & 78.00 & 333.00 & 392.76 & 40.00 & 0.90 & 80.00 & 17.00 \\
\hline 1917 & 341.70 & 107.60 & $31.49 \%$ & 234.10 & 233.20 & 0.90 & 253.18 & 79.00 & 28.60 & 155.14 & 78.00 & 333.00 & 392.77 & 40.00 & 0.90 & 80.00 & 17.00 \\
\hline 1918 & 341.70 & 107.58 & $31.48 \%$ & 234.12 & 233.22 & 0.90 & 253.18 & 79.00 & 28.58 & 155.16 & 78.00 & 333.00 & 392.79 & 40.00 & 0.90 & 80.00 & 17.00 \\
\hline 1919 & 341.70 & 107.56 & $31.48 \%$ & 234.14 & 233.24 & 0.90 & 253.19 & 79.00 & 28.56 & 155.18 & 78.00 & 333.00 & 392.81 & 40.00 & 0.90 & 80.00 & 17.00 \\
\hline 1920 & 341.70 & 107.54 & $31.47 \%$ & 234.16 & 233.26 & 0.90 & 253.19 & 79.00 & 28.54 & 155.20 & 78.00 & 333.00 & 392.83 & 40.00 & 0.90 & 80.00 & 17.00 \\
\hline 1921 & 341.70 & 107.52 & $31.46 \%$ & 234.18 & 233.28 & 0.90 & 253.20 & 79.00 & 28.52 & 155.22 & 78.00 & 333.00 & 392.85 & 40.00 & 0.90 & 80.00 & 17.00 \\
\hline 1922 & 341.70 & 107.49 & $31.46 \%$ & 234.21 & 233.31 & 0.90 & 253.21 & 79.00 & 28.49 & 155.25 & 78.00 & 333.00 & 392.88 & 40.00 & 0.90 & 80.00 & 17.00 \\
\hline 1923 & 341.70 & 107.46 & $31.45 \%$ & 234.24 & 233.34 & 0.90 & 253.22 & 79.00 & 28.46 & 155.28 & 78.00 & 333.00 & 392.91 & 40.00 & 0.90 & 80.00 & 17.00 \\
\hline 1924 & 341.70 & 107.43 & $31.44 \%$ & 234.27 & 233.37 & 0.90 & 253.22 & 79.00 & 28.43 & 155.31 & 78.00 & 333.00 & 392.94 & 40.00 & 0.90 & 80.00 & 17.00 \\
\hline 1925 & 341.70 & 107.39 & $31.43 \%$ & 234.31 & 233.41 & 0.90 & 253.23 & 79.00 & 28.39 & 155.35 & 78.00 & 333.00 & 392.98 & 40.00 & 0.90 & 80.00 & 17.00 \\
\hline 1926 & 341.70 & 107.36 & $31.42 \%$ & 234.34 & 233.44 & 0.90 & 253.24 & 79.00 & 28.36 & 155.38 & 78.00 & 333.00 & 393.01 & 40.00 & 0.90 & 80.00 & 17.00 \\
\hline 1927 & 341.70 & 107.31 & $31.41 \%$ & 234.39 & 233.49 & 0.90 & 253.26 & 79.00 & 28.31 & 155.43 & 78.00 & 333.00 & 393.06 & 40.00 & 0.90 & 80.00 & 17.00 \\
\hline 1928 & 341.70 & 107.27 & $31.39 \%$ & 234.43 & 233.53 & 0.90 & 253.27 & 79.00 & 28.27 & 155.47 & 78.00 & 333.00 & 393.10 & 40.00 & 0.90 & 80.00 & 17.00 \\
\hline 1929 & 341.70 & 107.22 & $31.38 \%$ & 234.48 & 233.58 & 0.90 & 253.28 & 79.00 & 28.22 & 155.51 & 78.00 & 333.00 & 393.15 & 40.00 & 0.90 & 80.00 & 17.00 \\
\hline 1930 & 341.70 & 107.18 & $31.37 \%$ & 234.52 & 233.62 & 0.90 & 253.29 & 79.00 & 28.18 & 155.56 & 78.00 & 333.00 & 393.19 & 40.00 & 0.90 & 80.00 & 17.00 \\
\hline 1931 & 341.70 & 107.13 & $31.35 \%$ & 234.57 & 233.67 & 0.90 & 253.31 & 79.00 & 28.13 & 155.61 & 78.00 & 333.00 & 393.24 & 40.00 & 0.90 & 80.00 & 17.00 \\
\hline 1932 & 341.70 & 107.07 & $31.34 \%$ & 234.63 & 233.73 & 0.90 & 253.32 & 79.00 & 28.07 & 155.66 & 78.00 & 333.00 & 393.29 & 40.00 & 0.90 & 80.00 & 17.00 \\
\hline 1933 & 341.70 & 107.02 & $31.32 \%$ & 234.68 & 233.78 & 0.90 & 253.34 & 79.00 & 28.02 & 155.72 & 78.00 & 333.00 & 393.35 & 40.00 & 0.90 & 80.00 & 17.00 \\
\hline 1934 & 341.70 & 106.96 & $31.30 \%$ & 234.74 & 233.84 & 0.90 & 253.35 & 79.00 & 27.96 & 155.78 & 78.00 & 333.00 & 393.41 & 40.00 & 0.90 & 80.00 & 17.00 \\
\hline 1935 & 341.70 & 106.90 & $31.28 \%$ & 234.80 & 233.90 & 0.90 & 253.37 & 79.00 & 27.90 & 155.84 & 78.00 & 333.00 & 393.47 & 40.00 & 0.90 & 80.00 & 17.00 \\
\hline 1936 & 341.70 & 106.84 & $31.27 \%$ & 234.86 & 233.96 & 0.90 & 253.38 & 79.00 & 27.84 & 155.90 & 78.00 & 333.00 & 393.53 & 40.00 & 0.90 & 80.00 & 17.00 \\
\hline 1937 & 341.70 & 106.77 & $31.25 \%$ & 234.93 & 234.03 & 0.90 & 253.40 & 79.00 & 27.77 & 155.97 & 78.00 & 333.00 & 393.60 & 40.00 & 0.90 & 80.00 & 17.00 \\
\hline 1938 & 341.70 & 106.71 & $31.23 \%$ & 234.99 & 234.09 & 0.90 & 253.42 & 79.00 & 27.71 & 156.03 & 78.00 & 333.00 & 393.66 & 40.00 & 0.90 & 80.00 & 17.00 \\
\hline 1939 & 341.70 & 106.64 & $31.21 \%$ & 235.06 & 234.16 & 0.90 & 253.44 & 79.00 & 27.64 & 156.10 & 78.00 & 333.00 & 393.73 & 40.00 & 0.90 & 80.00 & 17.00 \\
\hline 1940 & 341.70 & 106.57 & $31.19 \%$ & 235.13 & 234.23 & 0.90 & 253.46 & 79.00 & 27.57 & 156.17 & 78.00 & 333.00 & 393.80 & 40.00 & 0.90 & 80.00 & 17.00 \\
\hline 1941 & 341.70 & 106.50 & $31.17 \%$ & 235.20 & 234.30 & 0.90 & 253.48 & 79.00 & 27.50 & 156.24 & 78.00 & 333.00 & 393.87 & 40.00 & 0.90 & 80.00 & 17.00 \\
\hline
\end{tabular}




\section{Continued}

\begin{tabular}{|c|c|c|c|c|c|c|c|c|c|c|c|c|c|c|c|c|c|}
\hline 1942 & 341.70 & 106.43 & $31.15 \%$ & 235.27 & 234.37 & 0.90 & 253.50 & 79.00 & 27.43 & 156.31 & 78.00 & 333.00 & 393.94 & 40.00 & 0.90 & 80.00 & 17.00 \\
\hline 1943 & 341.70 & 106.35 & $31.12 \%$ & 235.35 & 234.45 & 0.90 & 253.52 & 79.00 & 27.35 & 156.39 & 78.00 & 333.00 & 394.02 & 40.00 & 0.90 & 80.00 & 17.00 \\
\hline 1944 & 341.70 & 106.28 & $31.10 \%$ & 35.42 & 234.52 & 0.90 & 253.54 & 79.00 & 27.28 & 156.46 & 78.00 & 333.00 & 394.09 & 40.00 & 0.90 & 80.00 & 17.00 \\
\hline 1945 & 341.70 & 106.20 & $31.08 \%$ & 235.50 & 234.60 & 0.90 & 253.56 & 79.00 & 27.20 & 156.54 & 78.00 & 333.00 & 394.17 & 40.00 & 0.90 & 80.00 & 17.00 \\
\hline 1946 & 341.70 & 106.13 & $31.06 \%$ & 235.57 & 234.67 & 0.90 & 253.58 & 79.00 & 27.13 & 156.61 & 78.00 & 333.00 & 394.24 & 40.00 & 0.90 & 80.00 & 17.00 \\
\hline 1947 & 341.70 & 106.13 & $31.06 \%$ & 235.57 & 234.67 & 0.90 & 253.58 & 79.00 & 27.13 & 156.61 & 78.00 & 333.00 & 394.24 & 40.00 & 0.90 & 80.00 & 17.00 \\
\hline 1948 & 341.70 & 106.13 & $31.06 \%$ & 235.57 & 234.67 & 0.90 & 253.58 & 79.00 & 27.13 & 156.61 & 78.00 & 333.00 & 394.24 & 40.00 & 0.90 & 80.00 & 17.00 \\
\hline 1949 & 341.70 & 106.13 & $31.06 \%$ & 235.57 & 234.67 & 0.90 & 253.58 & 79.00 & 27.13 & 156.61 & 78.00 & 333.00 & 394.24 & 40.00 & 0.90 & 80.00 & 17.00 \\
\hline 1950 & 341.70 & 106.13 & $31.06 \%$ & 235.57 & 234.67 & 0.90 & 253.58 & 79.00 & 27.13 & 156.61 & 78.00 & 333.00 & 394.24 & 40.00 & 0.90 & 80.00 & 17.00 \\
\hline 1951 & 41.70 & 106.13 & $31.06 \%$ & 235.57 & 234.67 & 0.90 & 253.58 & 79.00 & 27.13 & 156.61 & 78.00 & 333.00 & 394.24 & 40.00 & 0.90 & 80.00 & 17.00 \\
\hline 1952 & 341.70 & 106.13 & $31.06 \%$ & 235.57 & 234.67 & 0.90 & 253.58 & 79.00 & 27.13 & 156.61 & 78.00 & 333.00 & 394.24 & 40.00 & 0.90 & 80.00 & 17.00 \\
\hline 1953 & 41.70 & 106.13 & $31.06 \%$ & 235.57 & 234.67 & 0.90 & 253.58 & 79.00 & 27.13 & 156.61 & 78.00 & 333.00 & 394.24 & 40.00 & 0.90 & 80.00 & 17.00 \\
\hline 1954 & 341.70 & 106.13 & $31.06 \%$ & 235.57 & 234.67 & 0.90 & 253.58 & 79.00 & 27.13 & 156.61 & 78.00 & 333.00 & 394.24 & 40.00 & 0.90 & 80.00 & 17.00 \\
\hline 1955 & 41.70 & 106.13 & $31.06 \%$ & 235.57 & 234.67 & 0.90 & 253.58 & 79.00 & 27.13 & 156.61 & 78.00 & 3.00 & 394.24 & 40.00 & 0.90 & 80.00 & 17.00 \\
\hline 1956 & 341.70 & 106.13 & $31.06 \%$ & 235.57 & 234.67 & 0.90 & 253.58 & 79.00 & 27.13 & 156.61 & 78.00 & 333.00 & 394.24 & 40.00 & 0.90 & 80.00 & 17.00 \\
\hline 1957 & 341.70 & 106.13 & $31.06 \%$ & 235.57 & 234.67 & 0.90 & 253.58 & 79.00 & 27.13 & 156.61 & 78.00 & 3.00 & 394.24 & 40.00 & 0.90 & 80.00 & 17.00 \\
\hline 1958 & 341.70 & 106.13 & $31.06 \%$ & 235.57 & 234.67 & 0.90 & 253.58 & 79.00 & 27.13 & 156.61 & 78.00 & 333.00 & 394.24 & 40.00 & 0.90 & 80.00 & 17.00 \\
\hline 1959 & 341.70 & 106.13 & $31.06 \%$ & 235.57 & 234.67 & 0.90 & 253.58 & 79.00 & 27.13 & 156.61 & 78.00 & 333.00 & 394.24 & 40.00 & 0.90 & 80.00 & 17.00 \\
\hline 1960 & 341.70 & 106.13 & $31.06 \%$ & 235.57 & 234.67 & 0.90 & 253.58 & 79.00 & 27.13 & 156.61 & 78.00 & 333.00 & 394.24 & 40.00 & 0.90 & 80.00 & 17.00 \\
\hline 1961 & 341.70 & 106.13 & $31.06 \%$ & 235.57 & 234.67 & 0.90 & 253.58 & 79.00 & 27.13 & 156.61 & 78.00 & 333.00 & 394.24 & 40.00 & 0.90 & 80.00 & 17.00 \\
\hline 1962 & 341.70 & 106.13 & $31.06 \%$ & 235.57 & 234.67 & 0.90 & 253.58 & 79.00 & 27.13 & 156.61 & 78.00 & 333.00 & 394.24 & 40.00 & 0.90 & 80.00 & 17.00 \\
\hline 1963 & 341.70 & 106.13 & $31.06 \%$ & 235.57 & 234.67 & 0.90 & 253.58 & 79.00 & 27.13 & 156.61 & 78.00 & 333.00 & 394.24 & 40.00 & 0.90 & 80.00 & 17.00 \\
\hline 1964 & 341.70 & 106.13 & $31.06 \%$ & 235.57 & 234.67 & 0.90 & 253.58 & 79.00 & 27.13 & 156.61 & 78.00 & 3.00 & 394.24 & 40.00 & 0.90 & 80.00 & 17.00 \\
\hline 1965 & 341.70 & 106.13 & $31.06 \%$ & 235.57 & 234.67 & 0.90 & 253.58 & 79.00 & 27.13 & 156.61 & 78.00 & 333.00 & 394.24 & 40.00 & 0.90 & 80.00 & 17.00 \\
\hline 1966 & 341.70 & 106.13 & $31.06 \%$ & 235.57 & 234.67 & 0.90 & 253.58 & 79.00 & 27.13 & 156.61 & 78.00 & 333.00 & 394.24 & 40.00 & 0.90 & 80.00 & 17.00 \\
\hline 1967 & 341.70 & 106.13 & $31.06 \%$ & 235.57 & 234.67 & 0.90 & 253.58 & 79.00 & 27.13 & 156.61 & 78.00 & 333.00 & 394.24 & 40.00 & 0.90 & 80.00 & 17.00 \\
\hline 1968 & 341.70 & 106.13 & $31.06 \%$ & 235.57 & 234.67 & 0.90 & 253.58 & 79.00 & 27.13 & 156.61 & 78.00 & 333.00 & 394.24 & 40.00 & 0.90 & 80.00 & 17.00 \\
\hline 1969 & 341.70 & 106.13 & $31.06 \%$ & 235.57 & 234.67 & 0.90 & 253.58 & 79.00 & 27.13 & 156.61 & 78.00 & 333.00 & 394.24 & 40.00 & 0.90 & 80.00 & 17.00 \\
\hline 1970 & 341.70 & 106.13 & $31.06 \%$ & 235.57 & 234.67 & 0.90 & 253.58 & 79.00 & 27.13 & 156.61 & 78.00 & 333.00 & 394.24 & 40.00 & 0.90 & 80.00 & 17.00 \\
\hline 1971 & 341.70 & 106.13 & $31.06 \%$ & 235.57 & 234.67 & 0.90 & 253.58 & 79.00 & 27.13 & 156.61 & 78.00 & 333.00 & 394.24 & 40.00 & 0.90 & 80.00 & 17.00 \\
\hline 1972 & 341.70 & 106.13 & $31.06 \%$ & 235.57 & 234.67 & 0.90 & 253.58 & 79.00 & 27.13 & 156.61 & 78.00 & 333.00 & 394.24 & 40.00 & 0.90 & 80.00 & 17.00 \\
\hline 1973 & 341.70 & 106.13 & $31.06 \%$ & 235.57 & 234.67 & 0.90 & 253.58 & 79.00 & 27.13 & 156.61 & 78.00 & 333.00 & 394.24 & 40.00 & 0.90 & 80.00 & 17.00 \\
\hline 1974 & 341.70 & 106.13 & $31.06 \%$ & 235.57 & 234.67 & 0.90 & 253.58 & 79.00 & 27.13 & 156.61 & 78.00 & 333.00 & 394.24 & 40.00 & 0.90 & 80.00 & 17.00 \\
\hline 1975 & 341.70 & 106.13 & $31.06 \%$ & 235.57 & 234.67 & 0.90 & 253.58 & 79.00 & 27.13 & 156.61 & 78.00 & 333.00 & 394.24 & 40.00 & 0.90 & 80.00 & 17.00 \\
\hline 1976 & 341.70 & 106.13 & $31.06 \%$ & 235.57 & 234.67 & 0.90 & 253.58 & 79.00 & 27.13 & 156.61 & 78.00 & 333.00 & 394.24 & 40.00 & 0.90 & 80.00 & 17.00 \\
\hline 1977 & 341.70 & 106.13 & $31.06 \%$ & 235.57 & 234.67 & 0.90 & 253.58 & 79.00 & 27.13 & 156.61 & 78.00 & 333.00 & 394.24 & 40.00 & 0.90 & 80.00 & 17.00 \\
\hline 1978 & 341.70 & 106.13 & $31.06 \%$ & 235.57 & 234.67 & 0.90 & 253.58 & 79.00 & 27.13 & 156.61 & 78.00 & 333.00 & 394.24 & 40.00 & 0.90 & 80.00 & 17.00 \\
\hline
\end{tabular}




\section{Continued}

\begin{tabular}{|c|c|c|c|c|c|c|c|c|c|c|c|c|c|c|c|c|c|}
\hline 1979 & 341.70 & 106.13 & $31.06 \%$ & 235.57 & 234.67 & 0.90 & 253.58 & 79.00 & 27.13 & 156.61 & 78.00 & 333.00 & 394.24 & 40.00 & 0.90 & 80.00 & 17.00 \\
\hline 1980 & 341.70 & 106.13 & $31.06 \%$ & 235.57 & 234.67 & 0.90 & 253.58 & 79.00 & 27.13 & 156.61 & 78.00 & 333.00 & 394.24 & 40.00 & 0.90 & 80.00 & 17.0 \\
\hline 1981 & 341.70 & 06.13 & $.06 \%$ & 35.57 & 234.67 & 0.90 & 253.58 & 79.00 & 27.1264 & 156.6126 & 78.0000 & 333.00 & 394.24 & 40.00 & 0.9000 & 80.00 & 17.0 \\
\hline 982 & 41.70 & 6.05 & $1.04 \%$ & 35.65 & 234.75 & .90 & 253.60 & 79.00 & 27.0468 & 156.6922 & 78.0000 & 333.00 & 394.32 & 40.00 & 0.9000 & 80.00 & 17.0 \\
\hline 983 & 341.70 & 105.97 & $1.01 \%$ & 235.73 & 234.83 & 0.90 & 253.62 & 79.00 & 26.9694 & 156.7696 & 78.0000 & 333.00 & 394.40 & 40.00 & 0.9000 & 80.00 & 17.0 \\
\hline 1984 & 341.70 & 105.89 & $30.99 \%$ & 235.81 & 234.91 & 0.90 & 253.64 & 79.00 & 26.8880 & 156.8510 & 78.0000 & 333.00 & 394.48 & 40.00 & 0.9000 & 80.00 & 17.0 \\
\hline 1985 & 341.70 & 105.81 & $30.97 \%$ & 235.89 & 234.99 & 0.90 & 253.66 & 79.00 & 26.8089 & 156.9301 & 78.0000 & 333.00 & 394.56 & 40.00 & 0.9000 & 80.00 & 17.00 \\
\hline 1986 & 341.70 & 105.73 & $30.94 \%$ & 235.97 & 235.07 & 0.90 & 253.68 & 79.00 & 26.7259 & 157.0131 & 78.0000 & 333.00 & 394.64 & 40.00 & 0.9000 & 80.00 & 17.00 \\
\hline 987 & 341.70 & 105.65 & $30.92 \%$ & 236.05 & 235.15 & 0.90 & 253.71 & 79.00 & 26.6454 & 157.0936 & 78.0000 & 333.00 & 394.72 & 40.00 & 0.9000 & 80.00 & 17.0 \\
\hline 988 & 41.70 & 105.56 & $.89 \%$ & 36.14 & 235.24 & 0.90 & 253.73 & 79.00 & 26.5610 & 157.1780 & 78.0000 & 333.00 & 394.81 & 40.00 & 0.9000 & 80.00 & 17.0 \\
\hline 1989 & 341.70 & 105.48 & $30.87 \%$ & 236.22 & 235.32 & 0.90 & 253.75 & 79.00 & 26.4793 & 157.2597 & 78.0000 & 333.00 & 394.89 & 40.00 & 0.9000 & 80.00 & 17.00 \\
\hline 1990 & 341.70 & 105.39 & $30.84 \%$ & 236.31 & 235.41 & 0.90 & 253.77 & 79.00 & 26.3938 & 157.3452 & 78.0000 & 333.00 & 394.98 & 40.00 & 0.9000 & 80.00 & 17.00 \\
\hline 1991 & 41.70 & 55.31 & $30.82 \%$ & 36.39 & 235.49 & 0.90 & 253.80 & 79.00 & 26.3111 & 157.4279 & 78.0000 & 333.00 & 395.06 & 40.00 & 0.9000 & 80.00 & 17.0 \\
\hline 992 & 41.70 & 55.22 & $.79 \%$ & 36.48 & 235.58 & 0.90 & 253.82 & 79.00 & 26.2248 & 157.5142 & 78.0000 & 333.00 & 395.14 & 40.00 & 0.9000 & 80.00 & 17.0 \\
\hline 1993 & 41.70 & 105.14 & $0.77 \%$ & 36.56 & 235.66 & 0.90 & 253.84 & 79.00 & 26.1414 & 157.5976 & 78.0000 & 333.00 & 395.23 & 40.00 & 0.9000 & 80.00 & 17.0 \\
\hline 1994 & 341.70 & 105.05 & $30.74 \%$ & 236.65 & 235.75 & 0.90 & 253.87 & 79.00 & 26.0545 & 157.6845 & 78.0000 & 333.00 & 395.32 & 40.00 & 0.9000 & 80.00 & 17.0 \\
\hline 1995 & 341.70 & 4.97 & $30.72 \%$ & 36.73 & 235.83 & 90 & 253.89 & 79.00 & 25.9706 & 157.7684 & 78.0000 & 333.00 & 395.40 & 40.00 & 0.9000 & 80.00 & 17.0 \\
\hline 1996 & 41.70 & 4.88 & $.69 \%$ & 36.82 & 235.92 & 0.90 & 253.91 & 79.00 & 25.8833 & 157.8557 & 78.0000 & 333.00 & 395.49 & 40.00 & 0.9000 & 80.00 & 17.0 \\
\hline 1997 & 41.70 & 84.80 & $0.67 \%$ & 36.90 & 236.00 & 0.90 & 253.93 & 79.00 & 25.7992 & 157.9398 & 78.0000 & 333.00 & 395.57 & 40.00 & 0.9000 & 80.00 & 17.0 \\
\hline 1998 & 41.70 & 104.71 & $0.64 \%$ & 236.99 & 236.09 & 0.90 & 253.96 & 79.00 & 25.7118 & 158.0272 & 78.0000 & 333.00 & 395.66 & 40.00 & 0.9000 & 80.00 & 17.0 \\
\hline 1999 & 341.70 & 104.63 & $30.62 \%$ & 237.07 & 236.17 & 0.90 & 253.98 & 79.00 & 25.6277 & 158.1113 & 78.0000 & 333.00 & 395.74 & 40.00 & 0.9000 & 80.00 & 17.0 \\
\hline 2000 & 341.70 & 04.54 & $.59 \%$ & 37.16 & 236.26 & 90 & 254.00 & 79.00 & 25.5404 & 158.1986 & 78.0000 & 333.00 & 395.83 & 40.00 & 0.9000 & 80.00 & 17.0 \\
\hline 2001 & 41.70 & 104.46 & $.57 \%$ & 7.24 & 236.34 & 90 & 254.03 & 79.00 & 25.4565 & 158.2825 & 78.0000 & 333.00 & 395.91 & 40.00 & 0.9000 & 80.00 & 17.0 \\
\hline 2002 & 41.70 & 04.37 & $0.54 \%$ & 37.33 & 236.43 & 0.90 & 254.05 & 79.00 & 25.3696 & 158.3694 & 78.0000 & 333.00 & 396.00 & 40.00 & 0.9000 & 80.00 & 17.0 \\
\hline 2003 & 41.70 & 04.29 & $30.52 \%$ & 37.41 & 236.51 & 0.90 & 254.07 & 79.00 & 25.2863 & 158.4527 & 78.0000 & 333.00 & 396.08 & 40.00 & 0.9000 & 80.00 & 17.0 \\
\hline 2004 & 341.70 & 4.20 & $0.49 \%$ & 37.50 & 236.60 & 0.90 & 254.09 & 79.00 & 25.2000 & 158.5390 & 78.0000 & 333.00 & 396.17 & 40.00 & 0.9000 & 80.00 & 17.0 \\
\hline 2005 & 341.70 & 104.12 & $.47 \%$ & 37.58 & 236.68 & 90 & 254.12 & 79.00 & & & 78.0000 & 333.00 & 396.25 & 40.00 & 0.9000 & 80.00 & 17.0 \\
\hline 2006 & 41.70 & 104.01 & $.44 \%$ & 37.69 & 236.79 & 0.90 & 254.14 & 79.00 & 25.0127 & 158.7263 & 78.0000 & 333.00 & 396.36 & 40.00 & 0.9000 & 80.00 & 17.0 \\
\hline 2007 & 41.70 & 103.93 & $30.42 \%$ & 37.77 & 236.87 & 0.90 & 254.17 & 79.00 & 24.9331 & 158.8060 & 78.0000 & 333.00 & 396.44 & 40.00 & 0.9000 & 80.00 & 17.0 \\
\hline 2008 & 41.70 & 103.83 & $30.39 \%$ & 37.87 & 236.97 & .90 & 254.19 & 9.00 & 24.8314 & 158.9076 & 78.0000 & 333.00 & 396.54 & 40.00 & 0.9000 & 80.00 & 17.0 \\
\hline 2009 & 41.70 & 103.75 & $30.36 \%$ & 37.95 & 237.05 & 0.90 & 254.21 & 79.00 & 24.7549 & 158.9841 & 78.0000 & 333.00 & 396.61 & 40.00 & 0.9000 & 80.00 & 17.0 \\
\hline 2010 & 341.70 & 103.66 & $30.34 \%$ & 238.04 & 237.14 & 0.90 & 254.24 & 79.00 & 24.6564 & 159.0826 & 78.0000 & 333.00 & 396.71 & 40.00 & 0.9000 & 80.00 & 17.0 \\
\hline 2011 & 341.70 & 3.58 & $30.31 \%$ & 8.12 & 237 & 90 & 254.26 & 79.00 & 24.5831 & 159.1560 & 78.0000 & 333.00 & 396.79 & 40.00 & 0.9000 & 80.00 & 17.0 \\
\hline 2012 & 341.70 & 103.49 & $30.29 \%$ & 38.21 & 237.31 & 0.90 & 254.28 & 79.00 & 24.4878 & 159.2512 & 78.0000 & 333.00 & 396.88 & 40.00 & 0.9000 & 80.00 & 17.0 \\
\hline 2013 & 341.70 & 103.42 & $30.27 \%$ & 238.28 & 237.38 & 0.90 & 254.30 & 79.00 & 24.4178 & 159.3212 & 78.0000 & 333.00 & 396.95 & 40.00 & 0.9000 & 80.00 & 17.00 \\
\hline 2014 & 341.70 & 103.33 & $30.24 \%$ & 38.37 & 237.47 & 0.90 & 254.33 & 79.00 & 24.3259 & 159.4131 & 78.0000 & 333.00 & 397.04 & 40.00 & 0.9000 & 80.00 & 17.0 \\
\hline 2015 & 341.70 & 103.26 & $30.22 \%$ & 38.44 & 237.54 & 0.90 & 254.35 & 79.00 & 24.2593 & 159.4798 & 78.0000 & 333.00 & 397.11 & 40.00 & 0.9000 & 80.00 & 17.0 \\
\hline 2016 & 341.70 & 103.17 & $30.19 \%$ & 238.53 & 237.63 & 0.90 & 254.37 & 79.00 & 24.1698 & 159.5692 & 78.0000 & 333.00 & 397.20 & 40.00 & 0.9000 & 80.00 & 17.0 \\
\hline
\end{tabular}




\section{Continued}

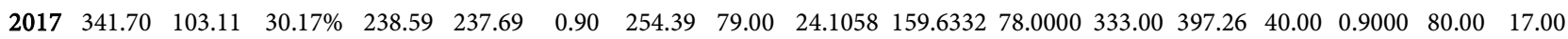

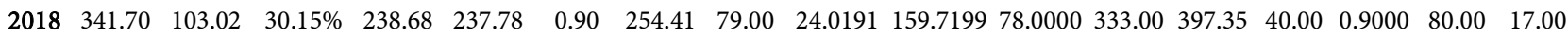

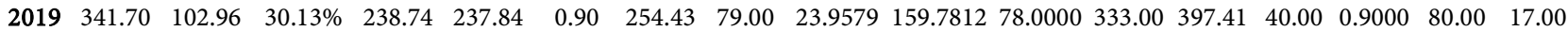

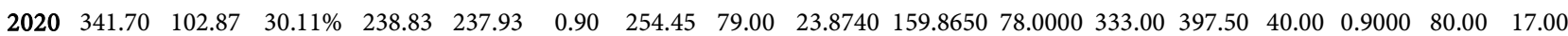

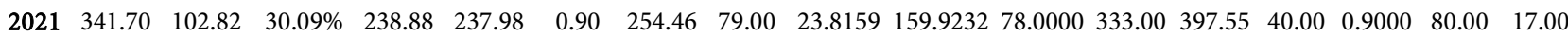

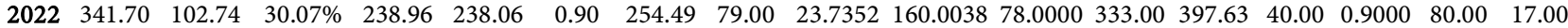

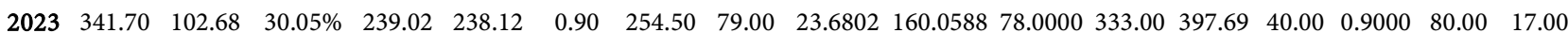

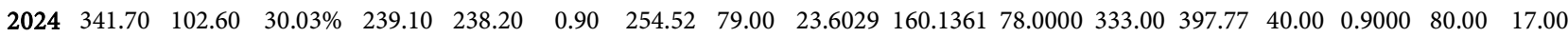

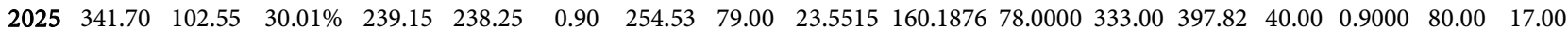

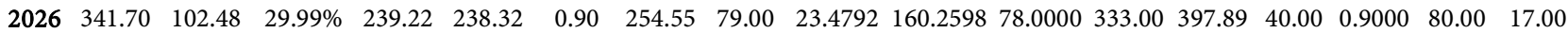

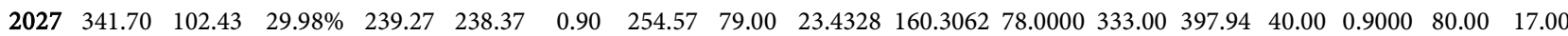

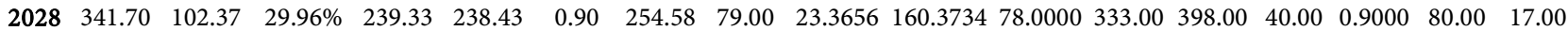

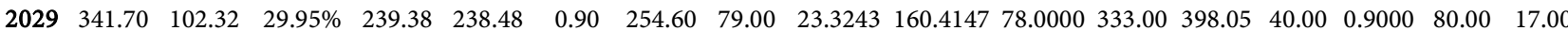

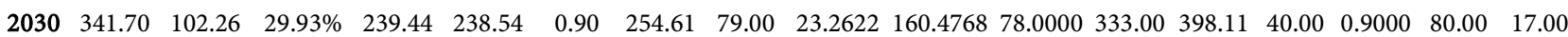

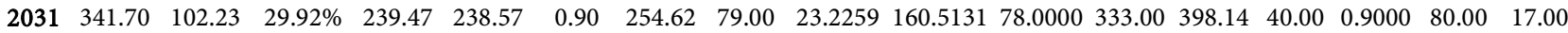

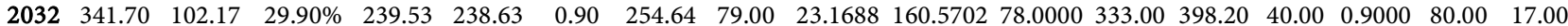

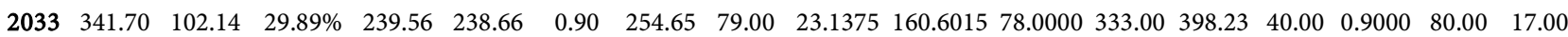

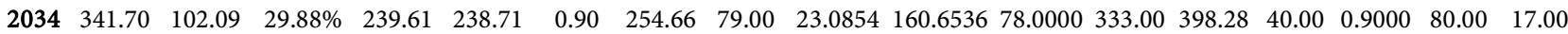

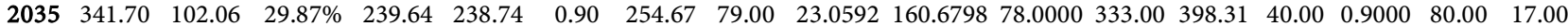

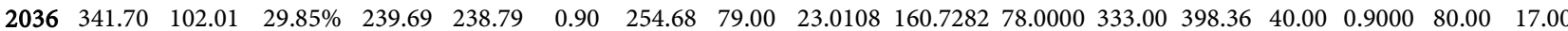

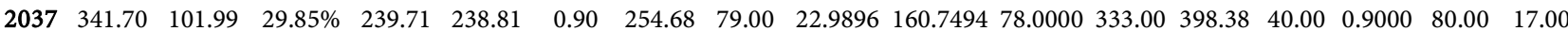

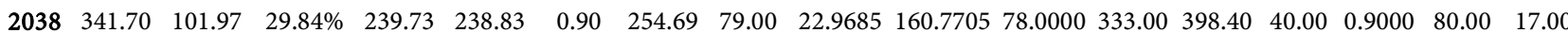

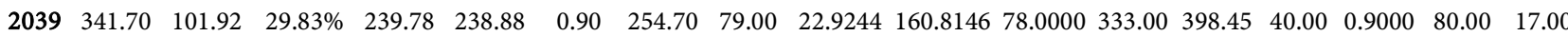

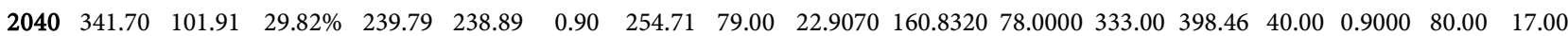

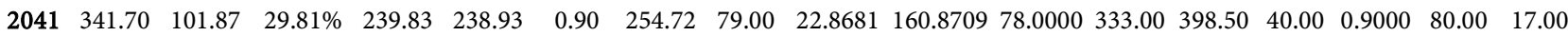

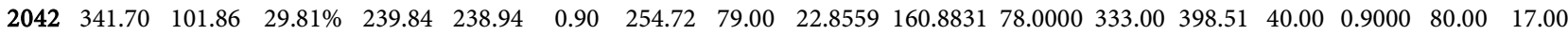

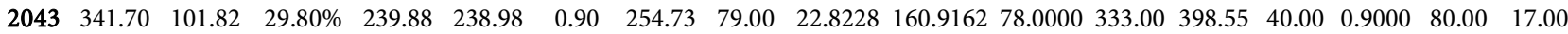

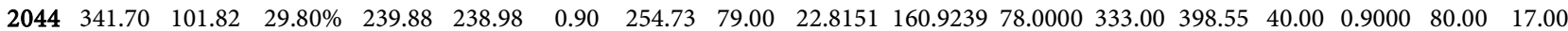

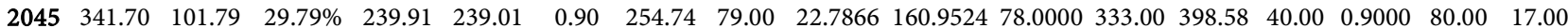

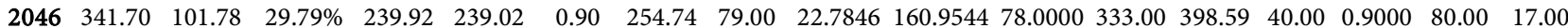

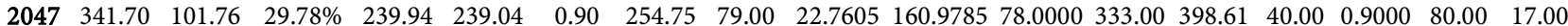

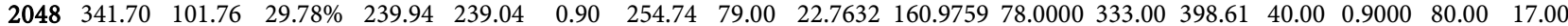

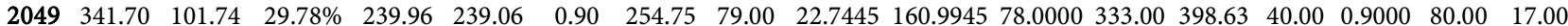

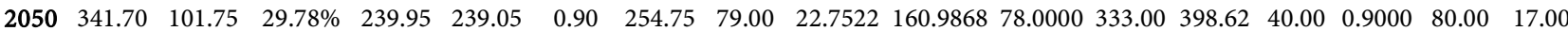

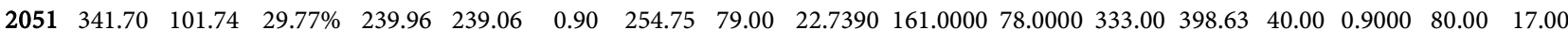

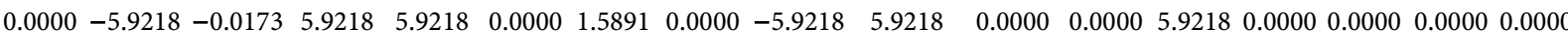


Table S2. Summary of temperature calculation.

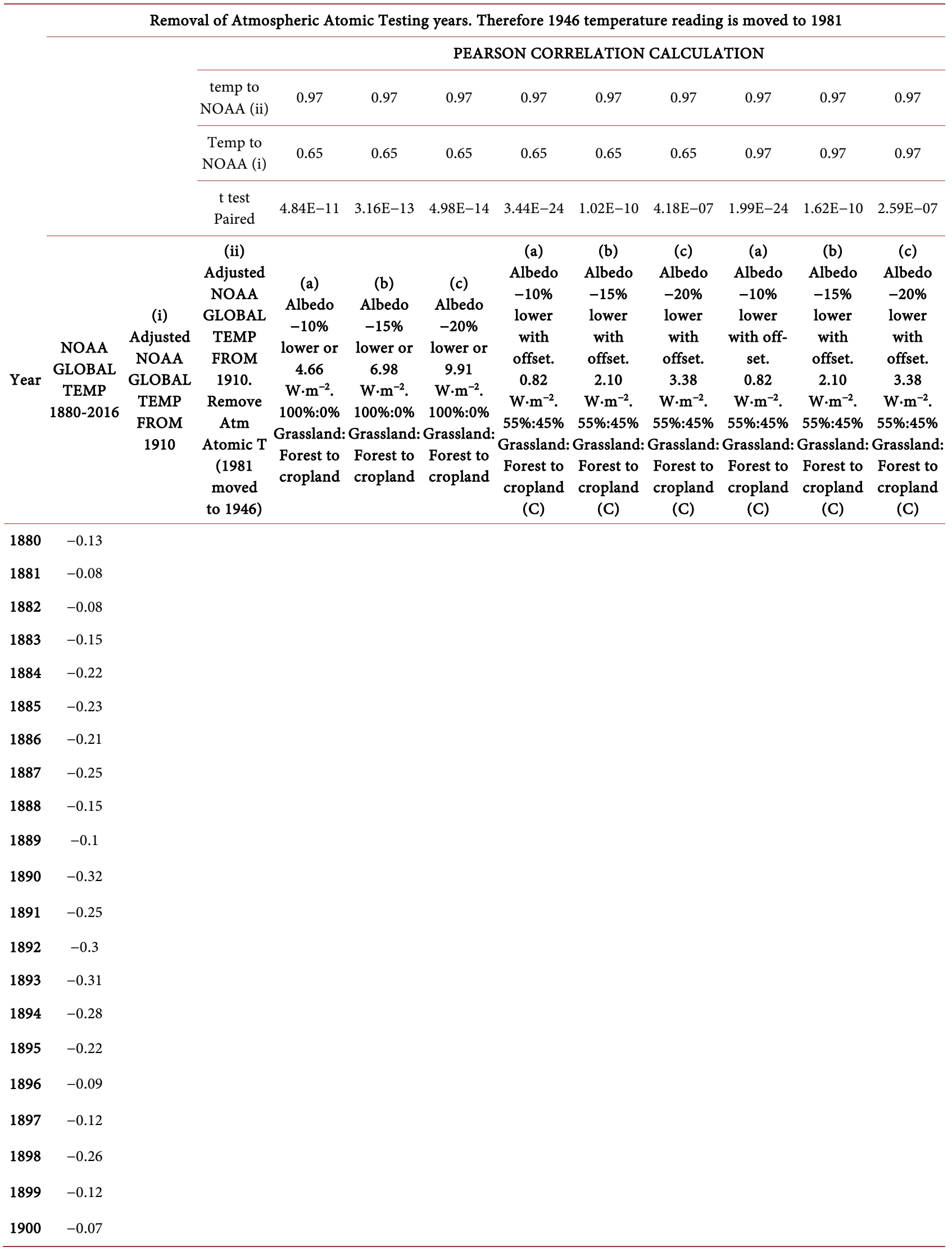


Continued

\begin{tabular}{|c|c|c|c|c|c|c|c|c|c|c|c|c|}
\hline 1901 & -0.14 & & & & & & & & & & & \\
\hline 1902 & -0.25 & & & & & & & & & & & \\
\hline 1903 & -0.34 & & & & & & & & & & & \\
\hline 1904 & -0.42 & & & & & & & & & & & \\
\hline 1905 & -0.29 & & & & & & & & & & & \\
\hline 1906 & -0.22 & & & & & & & & & & & \\
\hline 1907 & -0.37 & & & & & & & & & & & \\
\hline 1908 & -0.44 & & & & & & & & & & & \\
\hline 1909 & -0.43 & & & & & & & & & & & \\
\hline 1910 & -0.39 & 0.000 & 0.000 & 0.0 & 0.0 & 0.0 & 0.00 & 0.00 & 0.00 & 0.00 & 0.00 & 0.00 \\
\hline 1911 & -0.44 & -0.050 & -0.050 & 0.0 & 0.0 & 0.0 & 0.00 & 0.00 & 0.00 & 0.00 & 0.00 & 0.00 \\
\hline 1912 & -0.33 & 0.060 & 0.060 & 0.0 & 0.0 & 0.0 & 0.00 & 0.00 & 0.00 & 0.00 & 0.00 & 0.00 \\
\hline 1913 & -0.32 & 0.070 & 0.070 & 0.0 & 0.0 & 0.0 & 0.00 & 0.00 & 0.01 & 0.00 & 0.00 & 0.01 \\
\hline 1914 & -0.14 & 0.250 & 0.250 & 0.0 & 0.0 & 0.0 & 0.00 & 0.01 & 0.01 & 0.00 & 0.01 & 0.01 \\
\hline 1915 & -0.07 & 0.320 & 0.320 & 0.0 & 0.0 & 0.0 & 0.00 & 0.01 & 0.02 & 0.00 & 0.01 & 0.02 \\
\hline 1916 & -0.3 & 0.090 & 0.090 & 0.0 & 0.0 & 0.1 & 0.01 & 0.01 & 0.02 & 0.01 & 0.01 & 0.02 \\
\hline 1917 & -0.32 & 0.070 & 0.070 & 0.0 & 0.1 & 0.1 & 0.01 & 0.02 & 0.03 & 0.01 & 0.02 & 0.03 \\
\hline 1918 & -0.2 & 0.190 & 0.190 & 0.0 & 0.1 & 0.1 & 0.01 & 0.02 & 0.04 & 0.01 & 0.02 & 0.04 \\
\hline 1919 & -0.2 & 0.190 & 0.190 & 0.1 & 0.1 & 0.1 & 0.01 & 0.03 & 0.04 & 0.01 & 0.03 & 0.04 \\
\hline 1920 & -0.21 & 0.180 & 0.180 & 0.1 & 0.1 & 0.1 & 0.01 & 0.03 & 0.05 & 0.01 & 0.03 & 0.05 \\
\hline 1921 & -0.15 & 0.240 & 0.240 & 0.1 & 0.1 & 0.2 & 0.02 & 0.04 & 0.06 & 0.02 & 0.04 & 0.06 \\
\hline 1922 & -0.23 & 0.160 & 0.160 & 0.1 & 0.2 & 0.2 & 0.02 & 0.05 & 0.08 & 0.02 & 0.05 & 0.08 \\
\hline 1923 & -0.21 & 0.180 & 0.180 & 0.1 & 0.2 & 0.2 & 0.02 & 0.06 & 0.09 & 0.02 & 0.06 & 0.09 \\
\hline 1924 & -0.25 & 0.140 & 0.140 & 0.1 & 0.2 & 0.3 & 0.02 & 0.06 & 0.10 & 0.02 & 0.06 & 0.10 \\
\hline 1925 & -0.14 & 0.250 & 0.250 & 0.2 & 0.2 & 0.3 & 0.03 & 0.07 & 0.12 & 0.03 & 0.07 & 0.12 \\
\hline 1926 & -0.06 & 0.330 & 0.330 & 0.2 & 0.3 & 0.4 & 0.03 & 0.08 & 0.13 & 0.03 & 0.08 & 0.13 \\
\hline 1927 & -0.15 & 0.240 & 0.240 & 0.2 & 0.3 & 0.4 & 0.04 & 0.10 & 0.15 & 0.04 & 0.10 & 0.15 \\
\hline 1928 & -0.17 & 0.220 & 0.220 & 0.2 & 0.4 & 0.5 & 0.04 & 0.11 & 0.17 & 0.04 & 0.11 & 0.17 \\
\hline 1929 & -0.29 & 0.100 & 0.100 & 0.3 & 0.4 & 0.5 & 0.05 & 0.12 & 0.19 & 0.05 & 0.12 & 0.19 \\
\hline 1930 & -0.1 & 0.290 & 0.290 & 0.3 & 0.4 & 0.6 & 0.05 & 0.13 & 0.21 & 0.05 & 0.13 & 0.21 \\
\hline 1931 & -0.07 & 0.320 & 0.320 & 0.3 & 0.5 & 0.6 & 0.06 & 0.15 & 0.24 & 0.06 & 0.15 & 0.24 \\
\hline 1932 & -0.12 & 0.270 & 0.270 & 0.4 & 0.5 & 0.7 & 0.06 & 0.16 & 0.26 & 0.06 & 0.16 & 0.26 \\
\hline 1933 & -0.24 & 0.150 & 0.150 & 0.4 & 0.6 & 0.8 & 0.07 & 0.18 & 0.28 & 0.07 & 0.18 & 0.28 \\
\hline 1934 & -0.1 & 0.290 & 0.290 & 0.4 & 0.6 & 0.8 & 0.07 & 0.19 & 0.31 & 0.07 & 0.19 & 0.31 \\
\hline 1935 & -0.14 & 0.250 & 0.250 & 0.5 & 0.7 & 0.9 & 0.08 & 0.21 & 0.33 & 0.08 & 0.21 & 0.33 \\
\hline 1936 & -0.12 & 0.270 & 0.270 & 0.5 & 0.7 & 1.0 & 0.09 & 0.22 & 0.36 & 0.09 & 0.22 & 0.36 \\
\hline 1937 & -0.02 & 0.370 & 0.370 & 0.5 & 0.8 & 1.1 & 0.09 & 0.24 & 0.39 & 0.09 & 0.24 & 0.39 \\
\hline
\end{tabular}


Continued

\begin{tabular}{|c|c|c|c|c|c|c|c|c|c|c|c|c|}
\hline 1938 & -0.03 & 0.360 & 0.360 & 0.6 & 0.9 & 1.2 & 0.10 & 0.26 & 0.42 & 0.10 & 0.26 & 0.42 \\
\hline 1939 & -0.02 & 0.370 & 0.370 & 0.6 & 0.9 & 1.2 & 0.11 & 0.28 & 0.45 & 0.11 & 0.28 & 0.45 \\
\hline 1940 & 0.09 & 0.480 & 0.480 & 0.7 & 1.0 & 1.3 & 0.12 & 0.30 & 0.48 & 0.12 & 0.30 & 0.48 \\
\hline 1941 & 0.19 & 0.580 & 0.580 & 0.7 & 1.1 & 1.4 & 0.12 & 0.32 & 0.51 & 0.12 & 0.32 & 0.51 \\
\hline 1942 & 0.15 & 0.540 & 0.540 & 0.7 & 1.1 & 1.5 & 0.13 & 0.34 & 0.54 & 0.13 & 0.34 & 0.54 \\
\hline 1943 & 0.15 & 0.540 & 0.540 & 0.8 & 1.2 & 1.6 & 0.14 & 0.36 & 0.58 & 0.14 & 0.36 & 0.58 \\
\hline 1944 & 0.29 & 0.680 & 0.680 & 0.8 & 1.3 & 1.7 & 0.15 & 0.38 & 0.61 & 0.15 & 0.38 & 0.61 \\
\hline 1945 & 0.17 & 0.560 & 0.560 & 0.9 & 1.3 & 1.8 & 0.16 & 0.40 & 0.64 & 0.16 & 0.40 & 0.64 \\
\hline 1946 & -0.01 & 0.380 & 0.690 & 0.9 & 1.4 & 1.9 & 0.16 & 0.42 & 0.67 & 0.16 & 0.42 & 0.67 \\
\hline 1947 & -0.05 & 0.340 & 0.570 & 1.0 & 1.5 & 2.0 & 0.17 & 0.44 & 0.71 & & & \\
\hline 1948 & -0.06 & 0.330 & 0.730 & 1.0 & 1.5 & 2.0 & 0.18 & 0.46 & 0.74 & & & \\
\hline 1949 & -0.06 & 0.330 & 0.540 & 1.1 & 1.6 & 2.1 & 0.19 & 0.48 & 0.78 & & & \\
\hline 1950 & -0.16 & 0.230 & 0.520 & 1.1 & 1.7 & 2.2 & 0.20 & 0.51 & 0.81 & & & \\
\hline 1951 & -0.01 & 0.380 & 0.620 & 1.2 & 1.8 & 2.3 & 0.21 & 0.53 & 0.85 & & & \\
\hline 1952 & 0.03 & 0.420 & 0.760 & 1.2 & 1.8 & 2.4 & 0.21 & 0.55 & 0.89 & & & \\
\hline 1953 & 0.1 & 0.490 & 0.770 & 1.3 & 1.9 & 2.5 & 0.22 & 0.57 & 0.92 & & & \\
\hline 1954 & -0.11 & 0.280 & 0.680 & 1.3 & 2.0 & 2.6 & 0.23 & 0.59 & 0.96 & & & \\
\hline 1955 & -0.13 & 0.260 & 0.820 & 1.4 & 2.1 & 2.7 & 0.24 & 0.62 & 1.00 & & & \\
\hline 1956 & -0.2 & 0.190 & 0.790 & 1.4 & 2.1 & 2.8 & 0.25 & 0.64 & 1.03 & & & \\
\hline 1957 & 0.05 & 0.440 & 0.650 & 1.5 & 2.2 & 2.9 & 0.26 & 0.66 & 1.07 & & & \\
\hline 1958 & 0.11 & 0.500 & 0.670 & 1.5 & 2.3 & 3.0 & 0.27 & 0.69 & 1.11 & & & \\
\hline 1959 & 0.06 & 0.450 & 0.730 & 1.6 & 2.4 & 3.1 & 0.28 & 0.71 & 1.14 & & & \\
\hline 1960 & 0.02 & 0.410 & 0.850 & 1.6 & 2.4 & 3.2 & 0.29 & 0.73 & 1.18 & & & \\
\hline 1961 & 0.08 & 0.470 & 0.710 & 1.7 & 2.5 & 3.4 & 0.30 & 0.76 & 1.22 & & & \\
\hline 1962 & 0.09 & 0.480 & 0.910 & 1.7 & 2.6 & 3.5 & 0.30 & 0.78 & 1.25 & & & \\
\hline 1963 & 0.11 & 0.500 & 1.020 & 1.8 & 2.7 & 3.6 & 0.31 & 0.80 & 1.29 & & & \\
\hline 1964 & -0.15 & 0.240 & 0.830 & 1.8 & 2.7 & 3.7 & 0.32 & 0.83 & 1.33 & & & \\
\hline 1965 & -0.08 & 0.310 & 0.810 & 1.9 & 2.8 & 3.8 & 0.33 & 0.85 & 1.37 & & & \\
\hline 1966 & -0.02 & 0.370 & 0.930 & 1.9 & 2.9 & 3.9 & 0.34 & 0.87 & 1.40 & & & \\
\hline 1967 & -0.01 & 0.380 & 0.990 & 2.0 & 3.0 & 4.0 & 0.35 & 0.90 & 1.44 & & & \\
\hline 1968 & -0.03 & 0.360 & 1.000 & 2.0 & 3.1 & 4.1 & 0.36 & 0.92 & 1.48 & & & \\
\hline 1969 & 0.09 & 0.480 & 0.970 & 2.1 & 3.1 & 4.2 & 0.37 & 0.94 & 1.52 & & & \\
\hline 1970 & 0.04 & 0.430 & 1.050 & 2.1 & 3.2 & 4.3 & 0.38 & 0.96 & 1.55 & & & \\
\hline 1971 & -0.08 & 0.310 & 1.000 & 2.2 & 3.3 & 4.4 & 0.39 & 0.99 & 1.60 & & & \\
\hline 1972 & 0.03 & 0.420 & 1.000 & 2.2 & 3.4 & 4.5 & 0.40 & 1.01 & 1.63 & & & \\
\hline 1973 & 0.17 & 0.560 & 0.930 & 2.3 & 3.5 & 4.6 & 0.41 & 1.04 & 1.68 & & & \\
\hline 1974 & -0.07 & 0.320 & 1.020 & 2.4 & 3.5 & 4.7 & 0.42 & 1.06 & 1.71 & & & \\
\hline
\end{tabular}


Continued

\begin{tabular}{|c|c|c|c|c|c|c|c|c|c|c|c|c|}
\hline 1975 & 0 & 0.390 & 1.090 & 2.4 & 3.6 & 4.8 & 0.43 & 1.09 & 1.75 & & & \\
\hline 1976 & -0.08 & 0.310 & 0.970 & 2.5 & 3.7 & 4.9 & 0.43 & 1.11 & 1.78 & & & \\
\hline 1977 & 0.2 & 0.590 & 1.010 & 2.5 & 3.8 & 5.0 & 0.44 & 1.13 & 1.83 & & & \\
\hline 1978 & 0.11 & 0.500 & 1.060 & 2.6 & 3.8 & 5.1 & 0.45 & 1.15 & 1.86 & & & \\
\hline 1979 & 0.23 & 0.620 & 1.130 & 2.6 & 3.9 & 5.2 & 0.46 & 1.18 & 1.90 & & & \\
\hline 1980 & 0.27 & 0.660 & 1.290 & 2.7 & 4.0 & 5.3 & 0.47 & 1.20 & 1.93 & & & \\
\hline 1981 & 0.3 & 0.690 & 1.330 & 2.7 & 4.1 & 5.4 & 0.48 & 1.22 & 1.96 & 0.16 & 0.42 & 0.67 \\
\hline 1982 & 0.18 & 0.570 & & 2.7 & 4.1 & 5.5 & 0.48 & 1.24 & 1.99 & 0.17 & 0.44 & 0.71 \\
\hline 1983 & 0.34 & 0.730 & & 2.8 & 4.2 & 5.6 & 0.49 & 1.26 & 2.03 & 0.18 & 0.46 & 0.74 \\
\hline 1985 & 0.13 & 0.520 & & 2.9 & 4.3 & 5.8 & 0.51 & 1.30 & 2.09 & 0.20 & 0.51 & 0.81 \\
\hline 1986 & 0.23 & 0.620 & & 2.9 & 4.4 & 5.8 & 0.51 & 1.32 & 2.12 & 0.21 & 0.53 & 0.85 \\
\hline 1987 & 0.37 & 0.760 & & 3.0 & 4.4 & 5.9 & 0.52 & 1.34 & 2.15 & 0.21 & 0.55 & 0.89 \\
\hline 1988 & 0.38 & 0.770 & & 3.0 & 4.5 & 6.0 & 0.53 & 1.35 & 2.18 & 0.22 & 0.57 & 0.92 \\
\hline 1989 & 0.29 & 0.680 & & 3.0 & 4.6 & 6.1 & 0.54 & 1.37 & 2.21 & 0.23 & 0.59 & 0.96 \\
\hline 1990 & 0.43 & 0.820 & & 3.1 & 4.6 & 6.1 & 0.54 & 1.39 & 2.23 & 0.24 & 0.62 & 1.00 \\
\hline 1991 & 0.4 & 0.790 & & 3.1 & 4.7 & 6.2 & 0.55 & 1.41 & 2.26 & 0.25 & 0.64 & 1.03 \\
\hline 1992 & 0.26 & 0.650 & & 3.1 & 4.7 & 6.3 & 0.55 & 1.42 & 2.28 & 0.26 & 0.66 & 1.07 \\
\hline 1993 & 0.28 & 0.670 & & 3.2 & 4.8 & 6.4 & 0.56 & 1.44 & 2.31 & 0.27 & 0.69 & 1.11 \\
\hline 1995 & 0.46 & 0.850 & & 3.2 & 4.9 & 6.5 & 0.57 & 1.46 & 2.36 & 0.29 & 0.73 & 1.18 \\
\hline 1996 & 0.32 & 0.710 & & 3.3 & 4.9 & 6.5 & 0.58 & 1.47 & 2.37 & 0.30 & 0.76 & 1.22 \\
\hline 1997 & 0.52 & 0.910 & & 3.3 & 5.0 & 6.6 & 0.58 & 1.49 & 2.40 & 0.30 & 0.78 & 1.25 \\
\hline 1998 & 0.63 & 1.020 & & 3.3 & 5.0 & 6.6 & 0.59 & 1.50 & 2.41 & 0.31 & 0.80 & 1.29 \\
\hline 1999 & 0.44 & 0.830 & & 3.4 & 5.0 & 6.7 & 0.59 & 1.51 & 2.43 & 0.32 & 0.83 & 1.33 \\
\hline 2000 & 0.42 & 0.810 & & 3.4 & 5.1 & 6.7 & 0.59 & 1.52 & 2.45 & 0.33 & 0.85 & 1.37 \\
\hline 2001 & 0.54 & 0.930 & & 3.4 & 5.1 & 6.8 & 0.60 & 1.53 & 2.47 & 0.34 & 0.87 & 1.40 \\
\hline 2002 & 0.6 & 0.990 & & 3.4 & 5.1 & 6.8 & 0.60 & 1.54 & 2.48 & 0.35 & 0.90 & 1.44 \\
\hline 2003 & 0.61 & 1.000 & & 3.4 & 5.2 & 6.9 & 0.61 & 1.55 & 2.50 & 0.36 & 0.92 & 1.48 \\
\hline 2004 & 0.58 & 0.970 & & 3.4 & 5.2 & 6.9 & 0.61 & 1.55 & 2.50 & 0.37 & 0.94 & 1.52 \\
\hline 2005 & 0.66 & 1.050 & & 3.5 & 5.2 & 6.9 & 0.61 & 1.56 & 2.52 & 0.38 & 0.96 & 1.55 \\
\hline 2006 & 0.61 & 1.000 & & 3.5 & 5.2 & 7.0 & 0.61 & 1.57 & 2.52 & 0.39 & 0.99 & 1.60 \\
\hline 2007 & 0.61 & 1.000 & & 3.5 & 5.2 & 7.0 & 0.62 & 1.58 & 2.54 & 0.40 & 1.01 & 1.63 \\
\hline 2008 & 0.54 & 0.930 & & 3.5 & 5.2 & 7.0 & 0.62 & 1.58 & 2.54 & 0.41 & 1.04 & 1.68 \\
\hline 2009 & 0.63 & 1.020 & & 3.5 & 5.3 & 7.0 & 0.62 & 1.59 & 2.55 & 0.42 & 1.06 & 1.71 \\
\hline 2010 & 0.7 & 1.090 & & 3.5 & 5.3 & 7.0 & 0.62 & 1.59 & 2.56 & 0.43 & 1.09 & 1.75 \\
\hline 2011 & 0.58 & 0.970 & & 3.5 & 5.3 & 7.1 & 0.62 & 1.59 & 2.57 & 0.43 & 1.11 & 1.78 \\
\hline 2012 & 0.62 & 1.010 & & 3.5 & 5.3 & 7.1 & 0.62 & 1.59 & 2.56 & 0.44 & 1.13 & 1.83 \\
\hline 2013 & 0.67 & 1.060 & & 3.5 & 5.3 & 7.1 & 0.62 & 1.60 & 2.57 & 0.45 & 1.15 & 1.86 \\
\hline
\end{tabular}




\section{Continued}

\begin{tabular}{|c|c|c|c|c|c|c|c|c|c|c|c|}
\hline 2014 & 0.74 & 1.130 & 3.5 & 5.3 & 7.1 & 0.62 & 1.60 & 2.57 & 0.46 & 1.18 & 1.90 \\
\hline 2015 & 0.9 & 1.290 & 3.5 & 5.3 & 7.1 & 0.63 & 1.60 & 2.58 & 0.47 & 1.20 & 1.93 \\
\hline 2016 & 0.94 & 1.330 & 3.5 & 5.3 & 7.1 & 0.62 & 1.60 & 2.57 & 0.48 & 1.22 & 1.96 \\
\hline 2017 & & & & & & & & & 0.48 & 1.24 & 1.99 \\
\hline 2018 & & & & & & & & & 0.49 & 1.26 & 2.03 \\
\hline 2019 & & & & & & & & & 0.50 & 1.28 & 2.06 \\
\hline 2020 & & & & & & & & & 0.51 & 1.30 & 2.09 \\
\hline 2021 & & & & & & & & & 0.51 & 1.32 & 2.12 \\
\hline 2022 & & & & & & & & & 0.52 & 1.34 & 2.15 \\
\hline 2023 & & & & & & & & & 0.53 & 1.35 & 2.18 \\
\hline 2024 & & & & & & & & & 0.54 & 1.37 & 2.21 \\
\hline 2025 & & & & & & & & & 0.54 & 1.39 & 2.23 \\
\hline 2026 & & & & & & & & & 0.55 & 1.41 & 2.26 \\
\hline 2027 & & & & & & & & & 0.55 & 1.42 & 2.28 \\
\hline 2028 & & & & & & & & & 0.56 & 1.44 & 2.31 \\
\hline 2029 & & & & & & & & & 0.57 & 1.45 & 2.33 \\
\hline 2030 & & & & & & & & & 0.57 & 1.46 & 2.36 \\
\hline 2031 & & & & & & & & & 0.58 & 1.47 & 2.37 \\
\hline 2032 & & & & & & & & & 0.58 & 1.49 & 2.40 \\
\hline 2033 & & & & & & & & & 0.59 & 1.50 & 2.41 \\
\hline 2034 & & & & & & & & & 0.59 & 1.51 & 2.43 \\
\hline 2035 & & & & & & & & & 0.59 & 1.52 & 2.45 \\
\hline 2036 & & & & & & & & & 0.60 & 1.53 & 2.47 \\
\hline 2037 & & & & & & & & & 0.60 & 1.54 & 2.48 \\
\hline 2038 & & & & & & & & & 0.61 & 1.55 & 2.50 \\
\hline 2039 & & & & & & & & & 0.61 & 1.55 & 2.50 \\
\hline 2040 & & & & & & & & & 0.61 & 1.56 & 2.52 \\
\hline 2041 & & & & & & & & & 0.61 & 1.57 & 2.52 \\
\hline 2042 & & & & & & & & & 0.62 & 1.58 & 2.54 \\
\hline 2043 & & & & & & & & & 0.62 & 1.58 & 2.54 \\
\hline 2044 & & & & & & & & & 0.62 & 1.59 & 2.55 \\
\hline 2045 & & & & & & & & & 0.62 & 1.59 & 2.56 \\
\hline 2046 & & & & & & & & & 0.62 & 1.59 & 2.57 \\
\hline 2047 & & & & & & & & & 0.62 & 1.59 & 2.56 \\
\hline 2048 & & & & & & & & & 0.62 & 1.60 & 2.57 \\
\hline 2049 & & & & & & & & & 0.62 & 1.60 & 2.57 \\
\hline 2050 & & & & & & & & & 0.63 & 1.60 & 2.58 \\
\hline 2051 & & & & & & & & & 0.62 & 1.60 & 2.57 \\
\hline
\end{tabular}


Removal of Atmospheric Atomic Testing years. Therefore 1946 temperature reading is moved to 1981

ENERGY CHARTS (Total Joules)

$\begin{array}{llllll}\text { (d) Albedo-10\% } & \text { (e) Albedo -15\% } & \text { (f) Albedo -20\% } & \text { (d) Albedo -10\% } & \text { (e) Albedo -15\% } & \text { (f) Albedo -20\% }\end{array}$

Year lower with offset. $0.82 \mathrm{~W} \cdot \mathrm{m}^{-2}$.

55\%: $45 \%$

lower with offset.

lowerwith offset.

$2.10 \mathrm{~W} \cdot \mathrm{m}^{-2}$.

55\%: $45 \%$

$3.38 \mathrm{~W} \cdot \mathrm{m}^{-2}$.

55\%: $45 \%$

Grassland: Forest

Grassland: Forest

Grassland: Forest

lower with offset.

$0.82 \mathrm{~W} \cdot \mathrm{m}^{-2}$.

55\%: $45 \%$

lower with offset.

lower with offset.

to cropland (J)

to cropland (J)

to cropland (J)

Grassland: Forest

$2.10 \mathrm{~W} \cdot \mathrm{m}^{-2}$.

55\%: $45 \%$

$3.38 \mathrm{~W} \cdot \mathrm{m}^{-2}$.

55\%: $45 \%$

to cropland (J)

Grassland: Forest

Grassland: Forest

to cropland (J)

to cropland (J)

1880

1881

1882

1883

1884

1885

1886

1887

1888

1889

1890

1891

1892

1893

1894

1895

1896

1897

1898

1899

1900

1901

1902

1903

1904

1905

1906

1907

1908

1909

1910

$0.00 \mathrm{E}+00$

$0.00 \mathrm{E}+00$

$0.00 \mathrm{E}+00$

$0.00 \mathrm{E}+00$

$0.00 \mathrm{E}+00$

1911

$6.57 \mathrm{E}+19$

$1.68 \mathrm{E}+20$

$2.70 \mathrm{E}+20$

$6.57 \mathrm{E}+19$

$1.68 \mathrm{E}+20$

$2.70 \mathrm{E}+20$ 


\section{Continued}

\begin{tabular}{|c|c|c|c|c|c|c|}
\hline 1912 & $1.30 \mathrm{E}+20$ & $3.33 \mathrm{E}+20$ & $5.36 \mathrm{E}+20$ & $1.30 \mathrm{E}+20$ & $3.33 \mathrm{E}+20$ & $5.36 \mathrm{E}+20$ \\
\hline 1913 & $2.59 \mathrm{E}+20$ & $6.62 \mathrm{E}+20$ & $1.07 \mathrm{E}+21$ & $2.59 \mathrm{E}+20$ & $6.62 \mathrm{E}+20$ & $1.07 \mathrm{E}+21$ \\
\hline 1914 & $3.85 \mathrm{E}+20$ & $9.85 \mathrm{E}+20$ & $1.59 \mathrm{E}+21$ & $3.85 \mathrm{E}+20$ & $9.85 \mathrm{E}+20$ & $1.59 \mathrm{E}+21$ \\
\hline 1915 & $5.75 \mathrm{E}+20$ & $1.47 \mathrm{E}+21$ & $2.37 \mathrm{E}+21$ & $5.75 \mathrm{E}+20$ & $1.47 \mathrm{E}+21$ & $2.37 \mathrm{E}+21$ \\
\hline 1916 & $7.60 \mathrm{E}+20$ & $1.94 \mathrm{E}+21$ & $3.13 \mathrm{E}+21$ & $7.60 \mathrm{E}+20$ & $1.94 \mathrm{E}+21$ & $3.13 \mathrm{E}+21$ \\
\hline 1917 & $1.01 \mathrm{E}+21$ & $2.58 \mathrm{E}+21$ & $4.15 \mathrm{E}+21$ & $1.01 \mathrm{E}+21$ & $2.58 \mathrm{E}+21$ & $4.15 \mathrm{E}+21$ \\
\hline 1918 & $1.25 \mathrm{E}+21$ & $3.20 \mathrm{E}+21$ & $5.15 \mathrm{E}+21$ & $1.25 \mathrm{E}+21$ & $3.20 \mathrm{E}+21$ & $5.15 \mathrm{E}+21$ \\
\hline 1919 & $1.55 \mathrm{E}+21$ & $3.97 \mathrm{E}+21$ & $6.40 \mathrm{E}+21$ & $1.55 \mathrm{E}+21$ & $3.97 \mathrm{E}+21$ & $6.40 \mathrm{E}+21$ \\
\hline 1920 & $1.85 \mathrm{E}+21$ & $4.73 \mathrm{E}+21$ & $7.62 \mathrm{E}+21$ & $1.85 \mathrm{E}+21$ & $4.73 \mathrm{E}+21$ & $7.62 \mathrm{E}+21$ \\
\hline 1921 & $2.25 \mathrm{E}+21$ & $5.75 \mathrm{E}+21$ & $9.25 \mathrm{E}+21$ & $2.25 \mathrm{E}+21$ & $5.75 \mathrm{E}+21$ & $9.25 \mathrm{E}+21$ \\
\hline 1922 & $2.64 \mathrm{E}+21$ & $6.74 \mathrm{E}+21$ & $1.09 \mathrm{E}+22$ & $2.64 \mathrm{E}+21$ & $6.74 \mathrm{E}+21$ & $1.09 \mathrm{E}+22$ \\
\hline 1923 & $3.12 \mathrm{E}+21$ & $7.98 \mathrm{E}+21$ & $1.29 \mathrm{E}+22$ & $3.12 \mathrm{E}+21$ & $7.98 \mathrm{E}+21$ & $1.29 \mathrm{E}+22$ \\
\hline 1924 & $3.60 \mathrm{E}+21$ & $9.20 \mathrm{E}+21$ & $1.48 \mathrm{E}+22$ & $3.60 \mathrm{E}+21$ & $9.20 \mathrm{E}+21$ & $1.48 \mathrm{E}+22$ \\
\hline 1925 & $4.17 \mathrm{E}+21$ & $1.07 \mathrm{E}+22$ & $1.72 \mathrm{E}+22$ & $4.17 \mathrm{E}+21$ & $1.07 \mathrm{E}+22$ & $1.72 \mathrm{E}+22$ \\
\hline 1926 & $4.73 \mathrm{E}+21$ & $1.21 \mathrm{E}+22$ & $1.95 \mathrm{E}+22$ & $4.73 \mathrm{E}+21$ & $1.21 \mathrm{E}+22$ & $1.95 \mathrm{E}+22$ \\
\hline 1927 & $5.38 \mathrm{E}+21$ & $1.38 \mathrm{E}+22$ & $2.22 \mathrm{E}+22$ & $5.38 \mathrm{E}+21$ & $1.38 \mathrm{E}+22$ & $2.22 \mathrm{E}+22$ \\
\hline 1928 & $6.02 \mathrm{E}+21$ & $1.54 \mathrm{E}+22$ & $2.48 \mathrm{E}+22$ & $6.02 \mathrm{E}+21$ & $1.54 \mathrm{E}+22$ & $2.48 \mathrm{E}+22$ \\
\hline 1929 & $6.75 \mathrm{E}+21$ & $1.73 \mathrm{E}+22$ & $2.78 \mathrm{E}+22$ & $6.75 \mathrm{E}+21$ & $1.73 \mathrm{E}+22$ & $2.78 \mathrm{E}+22$ \\
\hline 1930 & $7.46 \mathrm{E}+21$ & $1.91 \mathrm{E}+22$ & $3.07 \mathrm{E}+22$ & $7.46 \mathrm{E}+21$ & $1.91 \mathrm{E}+22$ & $3.07 \mathrm{E}+22$ \\
\hline 1931 & $8.27 \mathrm{E}+21$ & $2.11 \mathrm{E}+22$ & $3.40 \mathrm{E}+22$ & $8.27 \mathrm{E}+21$ & $2.11 \mathrm{E}+22$ & $3.40 \mathrm{E}+22$ \\
\hline 1932 & $9.06 \mathrm{E}+21$ & $2.32 \mathrm{E}+22$ & $3.73 \mathrm{E}+22$ & $9.06 \mathrm{E}+21$ & $2.32 \mathrm{E}+22$ & $3.73 \mathrm{E}+22$ \\
\hline 1933 & $9.94 \mathrm{E}+21$ & $2.54 \mathrm{E}+22$ & $4.09 \mathrm{E}+22$ & $9.94 \mathrm{E}+21$ & $2.54 \mathrm{E}+22$ & $4.09 \mathrm{E}+22$ \\
\hline 1934 & $1.08 \mathrm{E}+22$ & $2.76 \mathrm{E}+22$ & $4.45 \mathrm{E}+22$ & $1.08 \mathrm{E}+22$ & $2.76 \mathrm{E}+22$ & $4.45 \mathrm{E}+22$ \\
\hline 1935 & $1.18 \mathrm{E}+22$ & $3.00 \mathrm{E}+22$ & $4.84 \mathrm{E}+22$ & $1.18 \mathrm{E}+22$ & $3.00 \mathrm{E}+22$ & $4.84 \mathrm{E}+22$ \\
\hline 1936 & $1.27 \mathrm{E}+22$ & $3.24 \mathrm{E}+22$ & $5.22 \mathrm{E}+22$ & $1.27 \mathrm{E}+22$ & $3.24 \mathrm{E}+22$ & $5.22 \mathrm{E}+22$ \\
\hline 1937 & $1.37 \mathrm{E}+22$ & $3.50 \mathrm{E}+22$ & $5.64 \mathrm{E}+22$ & $1.37 \mathrm{E}+22$ & $3.50 \mathrm{E}+22$ & $5.64 \mathrm{E}+22$ \\
\hline 1938 & $1.47 \mathrm{E}+22$ & $3.76 \mathrm{E}+22$ & $6.05 \mathrm{E}+22$ & $1.47 \mathrm{E}+22$ & $3.76 \mathrm{E}+22$ & $6.05 \mathrm{E}+22$ \\
\hline 1939 & $1.58 \mathrm{E}+22$ & $4.03 \mathrm{E}+22$ & $6.49 \mathrm{E}+22$ & $1.58 \mathrm{E}+22$ & $4.03 \mathrm{E}+22$ & $6.49 \mathrm{E}+22$ \\
\hline 1940 & $1.68 \mathrm{E}+22$ & $4.30 \mathrm{E}+22$ & $6.92 \mathrm{E}+22$ & $1.68 \mathrm{E}+22$ & $4.30 \mathrm{E}+22$ & $6.92 \mathrm{E}+22$ \\
\hline 1941 & $1.79 \mathrm{E}+22$ & $4.59 \mathrm{E}+22$ & $7.39 \mathrm{E}+22$ & $1.79 \mathrm{E}+22$ & $4.59 \mathrm{E}+22$ & $7.39 \mathrm{E}+22$ \\
\hline 1942 & $1.90 \mathrm{E}+22$ & $4.87 \mathrm{E}+22$ & $7.84 \mathrm{E}+22$ & $1.90 \mathrm{E}+22$ & $4.87 \mathrm{E}+22$ & $7.84 \mathrm{E}+22$ \\
\hline 1943 & $2.02 \mathrm{E}+22$ & $5.16 \mathrm{E}+22$ & $8.31 \mathrm{E}+22$ & $2.02 \mathrm{E}+22$ & $5.16 \mathrm{E}+22$ & $8.31 \mathrm{E}+22$ \\
\hline 1944 & $2.13 \mathrm{E}+22$ & $5.45 \mathrm{E}+22$ & $8.78 \mathrm{E}+22$ & $2.13 \mathrm{E}+22$ & $5.45 \mathrm{E}+22$ & $8.78 \mathrm{E}+22$ \\
\hline 1945 & $2.25 \mathrm{E}+22$ & $5.76 \mathrm{E}+22$ & $9.27 \mathrm{E}+22$ & $2.25 \mathrm{E}+22$ & $5.76 \mathrm{E}+22$ & $9.27 \mathrm{E}+22$ \\
\hline 1946 & $2.37 \mathrm{E}+22$ & $6.05 \mathrm{E}+22$ & $9.75 \mathrm{E}+22$ & $2.37 \mathrm{E}+22$ & $6.05 \mathrm{E}+22$ & $9.75 \mathrm{E}+22$ \\
\hline 1947 & $2.49 \mathrm{E}+22$ & $6.37 \mathrm{E}+22$ & $1.03 \mathrm{E}+23$ & & & \\
\hline 1948 & $2.61 \mathrm{E}+22$ & $6.67 \mathrm{E}+22$ & $1.07 \mathrm{E}+23$ & & & \\
\hline
\end{tabular}




\section{Continued}

\begin{tabular}{|c|c|c|c|c|c|c|}
\hline 1949 & $2.73 \mathrm{E}+22$ & $6.99 \mathrm{E}+22$ & $1.13 \mathrm{E}+23$ & & & \\
\hline 1950 & $2.86 \mathrm{E}+22$ & $7.30 \mathrm{E}+22$ & $1.18 \mathrm{E}+23$ & & & \\
\hline 1951 & $2.98 \mathrm{E}+22$ & $7.63 \mathrm{E}+22$ & $1.23 \mathrm{E}+23$ & & & \\
\hline 1952 & $3.11 \mathrm{E}+22$ & $7.94 \mathrm{E}+22$ & $1.28 \mathrm{E}+23$ & & & \\
\hline 1953 & $3.24 \mathrm{E}+22$ & $8.28 \mathrm{E}+22$ & $1.33 \mathrm{E}+23$ & & & \\
\hline 1954 & $3.36 \mathrm{E}+22$ & $8.60 \mathrm{E}+22$ & $1.38 \mathrm{E}+23$ & & & \\
\hline 1955 & $3.49 \mathrm{E}+22$ & $8.93 \mathrm{E}+22$ & $1.44 \mathrm{E}+23$ & & & \\
\hline 1956 & $3.62 \mathrm{E}+22$ & $9.26 \mathrm{E}+22$ & $1.49 \mathrm{E}+23$ & & & \\
\hline 1957 & $3.75 \mathrm{E}+22$ & $9.60 \mathrm{E}+22$ & $1.55 \mathrm{E}+23$ & & & \\
\hline 1958 & $3.88 \mathrm{E}+22$ & $9.92 \mathrm{E}+22$ & $1.60 \mathrm{E}+23$ & & & \\
\hline 1959 & $4.01 \mathrm{E}+22$ & $1.03 \mathrm{E}+23$ & $1.65 \mathrm{E}+23$ & & & \\
\hline 1960 & $4.14 \mathrm{E}+22$ & $1.06 \mathrm{E}+23$ & $1.71 \mathrm{E}+23$ & & & \\
\hline 1961 & $4.28 \mathrm{E}+22$ & $1.09 \mathrm{E}+23$ & $1.76 \mathrm{E}+23$ & & & \\
\hline 1962 & $4.41 \mathrm{E}+22$ & $1.13 \mathrm{E}+23$ & $1.81 \mathrm{E}+23$ & & & \\
\hline 1963 & $4.54 \mathrm{E}+22$ & $1.16 \mathrm{E}+23$ & $1.87 \mathrm{E}+23$ & & & \\
\hline 1964 & $4.67 \mathrm{E}+22$ & $1.19 \mathrm{E}+23$ & $1.92 \mathrm{E}+23$ & & & \\
\hline 1965 & $4.80 \mathrm{E}+22$ & $1.23 \mathrm{E}+23$ & $1.98 \mathrm{E}+23$ & & & \\
\hline 1966 & $4.93 \mathrm{E}+22$ & $1.26 \mathrm{E}+23$ & $2.03 \mathrm{E}+23$ & & & \\
\hline 1967 & $5.06 \mathrm{E}+22$ & $1.29 \mathrm{E}+23$ & $2.08 \mathrm{E}+23$ & & & \\
\hline 1968 & $5.19 \mathrm{E}+22$ & $1.33 \mathrm{E}+23$ & $2.14 \mathrm{E}+23$ & & & \\
\hline 1969 & $5.32 \mathrm{E}+22$ & $1.36 \mathrm{E}+23$ & $2.19 \mathrm{E}+23$ & & & \\
\hline 1970 & $5.45 \mathrm{E}+22$ & $1.39 \mathrm{E}+23$ & $2.24 \mathrm{E}+23$ & & & \\
\hline 1971 & $5.61 \mathrm{E}+22$ & $1.43 \mathrm{E}+23$ & $2.31 \mathrm{E}+23$ & & & \\
\hline 1972 & $5.73 \mathrm{E}+22$ & $1.47 \mathrm{E}+23$ & $2.36 \mathrm{E}+23$ & & & \\
\hline 1973 & $5.89 \mathrm{E}+22$ & $1.51 \mathrm{E}+23$ & $2.42 \mathrm{E}+23$ & & & \\
\hline 1974 & $6.00 \mathrm{E}+22$ & $1.54 \mathrm{E}+23$ & $2.47 \mathrm{E}+23$ & & & \\
\hline 1975 & $6.15 \mathrm{E}+22$ & $1.57 \mathrm{E}+23$ & $2.53 \mathrm{E}+23$ & & & \\
\hline 1976 & $6.27 \mathrm{E}+22$ & $1.60 \mathrm{E}+23$ & $2.58 \mathrm{E}+23$ & & & \\
\hline 1977 & $6.41 \mathrm{E}+22$ & $1.64 \mathrm{E}+23$ & $2.64 \mathrm{E}+23$ & & & \\
\hline 1978 & $6.52 \mathrm{E}+22$ & $1.67 \mathrm{E}+23$ & $2.68 \mathrm{E}+23$ & & & \\
\hline 1979 & $6.66 \mathrm{E}+22$ & $1.70 \mathrm{E}+23$ & $2.74 \mathrm{E}+23$ & & & \\
\hline 1980 & $6.76 \mathrm{E}+22$ & $1.73 \mathrm{E}+23$ & $2.78 \mathrm{E}+23$ & & & \\
\hline 1981 & $6.90 \mathrm{E}+22$ & $1.76 \mathrm{E}+23$ & $2.84 \mathrm{E}+23$ & $2.37 \mathrm{E}+22$ & $6.05 \mathrm{E}+22$ & $9.75 \mathrm{E}+22$ \\
\hline 1982 & $6.99 \mathrm{E}+22$ & $1.79 \mathrm{E}+23$ & $2.88 \mathrm{E}+23$ & $2.49 \mathrm{E}+22$ & $6.37 \mathrm{E}+22$ & $1.03 \mathrm{E}+23$ \\
\hline 1983 & $7.13 \mathrm{E}+22$ & $1.82 \mathrm{E}+23$ & $2.93 \mathrm{E}+23$ & $2.61 \mathrm{E}+22$ & $6.67 \mathrm{E}+22$ & $1.07 \mathrm{E}+23$ \\
\hline 1984 & $7.22 \mathrm{E}+22$ & $1.85 \mathrm{E}+23$ & $2.97 \mathrm{E}+23$ & $2.73 \mathrm{E}+22$ & $6.99 \mathrm{E}+22$ & $1.13 \mathrm{E}+23$ \\
\hline 1985 & $7.35 E+22$ & $1.88 \mathrm{E}+23$ & $3.02 \mathrm{E}+23$ & $2.86 \mathrm{E}+22$ & $7.30 \mathrm{E}+22$ & $1.18 \mathrm{E}+23$ \\
\hline
\end{tabular}




\section{Continued}

\begin{tabular}{|c|c|c|c|c|c|c|}
\hline 1986 & $7.44 \mathrm{E}+22$ & $1.90 \mathrm{E}+23$ & $3.06 \mathrm{E}+23$ & $2.98 \mathrm{E}+22$ & $7.63 \mathrm{E}+22$ & $1.23 \mathrm{E}+23$ \\
\hline 1987 & $7.56 \mathrm{E}+22$ & $1.93 \mathrm{E}+23$ & $3.11 \mathrm{E}+23$ & $3.11 \mathrm{E}+22$ & $7.94 \mathrm{E}+22$ & $1.28 \mathrm{E}+23$ \\
\hline 1988 & $7.64 \mathrm{E}+22$ & $1.95 \mathrm{E}+23$ & $3.15 \mathrm{E}+23$ & $3.24 \mathrm{E}+22$ & $8.28 \mathrm{E}+22$ & $1.33 \mathrm{E}+23$ \\
\hline 1989 & $7.76 \mathrm{E}+22$ & $1.98 \mathrm{E}+23$ & $3.19 \mathrm{E}+23$ & $3.36 \mathrm{E}+22$ & $8.60 \mathrm{E}+22$ & $1.38 \mathrm{E}+23$ \\
\hline 1990 & $7.84 \mathrm{E}+22$ & $2.00 \mathrm{E}+23$ & $3.23 \mathrm{E}+23$ & $3.49 \mathrm{E}+22$ & $8.93 \mathrm{E}+22$ & $1.44 \mathrm{E}+23$ \\
\hline 1991 & $7.95 \mathrm{E}+22$ & $2.03 \mathrm{E}+23$ & $3.27 \mathrm{E}+23$ & $3.62 \mathrm{E}+22$ & $9.26 \mathrm{E}+22$ & $1.49 \mathrm{E}+23$ \\
\hline 1992 & $8.02 \mathrm{E}+22$ & $2.05 \mathrm{E}+23$ & $3.30 \mathrm{E}+23$ & $3.75 \mathrm{E}+22$ & $9.60 \mathrm{E}+22$ & $1.55 \mathrm{E}+23$ \\
\hline 1993 & $8.12 \mathrm{E}+22$ & $2.08 \mathrm{E}+23$ & $3.34 \mathrm{E}+23$ & $3.88 \mathrm{E}+22$ & $9.92 \mathrm{E}+22$ & $1.60 \mathrm{E}+23$ \\
\hline 1994 & $8.18 \mathrm{E}+22$ & $2.09 \mathrm{E}+23$ & $3.37 \mathrm{E}+23$ & $4.01 \mathrm{E}+22$ & $1.03 \mathrm{E}+23$ & $1.65 \mathrm{E}+23$ \\
\hline 1995 & $8.28 \mathrm{E}+22$ & $2.12 \mathrm{E}+23$ & $3.41 \mathrm{E}+23$ & $4.14 \mathrm{E}+22$ & $1.06 \mathrm{E}+23$ & $1.71 \mathrm{E}+23$ \\
\hline 1996 & $8.33 \mathrm{E}+22$ & $2.13 E+23$ & $3.43 \mathrm{E}+23$ & $4.28 \mathrm{E}+22$ & $1.09 \mathrm{E}+23$ & $1.76 \mathrm{E}+23$ \\
\hline 1997 & $8.42 \mathrm{E}+22$ & $2.15 \mathrm{E}+23$ & $3.47 \mathrm{E}+23$ & $4.41 \mathrm{E}+22$ & $1.13 \mathrm{E}+23$ & $1.81 \mathrm{E}+23$ \\
\hline 1998 & $8.47 \mathrm{E}+22$ & $2.17 \mathrm{E}+23$ & $3.49 \mathrm{E}+23$ & $4.54 \mathrm{E}+22$ & $1.16 \mathrm{E}+23$ & $1.87 \mathrm{E}+23$ \\
\hline 1999 & $8.55 \mathrm{E}+22$ & $2.19 \mathrm{E}+23$ & $3.52 \mathrm{E}+23$ & $4.67 \mathrm{E}+22$ & $1.19 \mathrm{E}+23$ & $1.92 \mathrm{E}+23$ \\
\hline 2000 & $8.59 \mathrm{E}+22$ & $2.20 \mathrm{E}+23$ & $3.54 \mathrm{E}+23$ & $4.80 \mathrm{E}+22$ & $1.23 \mathrm{E}+23$ & $1.98 \mathrm{E}+23$ \\
\hline 2001 & $8.66 \mathrm{E}+22$ & $2.21 \mathrm{E}+23$ & $3.57 \mathrm{E}+23$ & $4.93 \mathrm{E}+22$ & $1.26 \mathrm{E}+23$ & $2.03 \mathrm{E}+23$ \\
\hline 2002 & $8.69 \mathrm{E}+22$ & $2.22 \mathrm{E}+23$ & $3.58 \mathrm{E}+23$ & $5.06 \mathrm{E}+22$ & $1.29 \mathrm{E}+23$ & $2.08 \mathrm{E}+23$ \\
\hline 2003 & $8.76 \mathrm{E}+22$ & $2.24 \mathrm{E}+23$ & $3.61 \mathrm{E}+23$ & $5.19 \mathrm{E}+22$ & $1.33 \mathrm{E}+23$ & $2.14 \mathrm{E}+23$ \\
\hline 2004 & $8.79 \mathrm{E}+22$ & $2.25 \mathrm{E}+23$ & $3.62 \mathrm{E}+23$ & $5.32 \mathrm{E}+22$ & $1.36 \mathrm{E}+23$ & $2.19 \mathrm{E}+23$ \\
\hline 2005 & $8.85 \mathrm{E}+22$ & $2.26 \mathrm{E}+23$ & $3.64 \mathrm{E}+23$ & $5.45 \mathrm{E}+22$ & $1.39 \mathrm{E}+23$ & $2.24 \mathrm{E}+23$ \\
\hline 2006 & $8.86 \mathrm{E}+22$ & $2.27 \mathrm{E}+23$ & $3.65 \mathrm{E}+23$ & $5.61 \mathrm{E}+22$ & $1.43 \mathrm{E}+23$ & $2.31 \mathrm{E}+23$ \\
\hline 2007 & $8.91 \mathrm{E}+22$ & $2.28 \mathrm{E}+23$ & $3.67 \mathrm{E}+23$ & $5.73 \mathrm{E}+22$ & $1.47 \mathrm{E}+23$ & $2.36 \mathrm{E}+23$ \\
\hline 2008 & $8.93 \mathrm{E}+22$ & $2.28 \mathrm{E}+23$ & $3.67 \mathrm{E}+23$ & $5.89 \mathrm{E}+22$ & $1.51 \mathrm{E}+23$ & $2.42 \mathrm{E}+23$ \\
\hline 2009 & $8.97 \mathrm{E}+22$ & $2.29 \mathrm{E}+23$ & $3.69 \mathrm{E}+23$ & $6.00 \mathrm{E}+22$ & $1.54 \mathrm{E}+23$ & $2.47 \mathrm{E}+23$ \\
\hline 2010 & $8.97 \mathrm{E}+22$ & $2.29 \mathrm{E}+23$ & $3.69 \mathrm{E}+23$ & $6.15 \mathrm{E}+22$ & $1.57 \mathrm{E}+23$ & $2.53 \mathrm{E}+23$ \\
\hline 2011 & $9.01 \mathrm{E}+22$ & $2.30 \mathrm{E}+23$ & $3.71 \mathrm{E}+23$ & $6.27 \mathrm{E}+22$ & $1.60 \mathrm{E}+23$ & $2.58 \mathrm{E}+23$ \\
\hline 2012 & $9.00 \mathrm{E}+22$ & $2.30 \mathrm{E}+23$ & $3.71 \mathrm{E}+23$ & $6.41 \mathrm{E}+22$ & $1.64 \mathrm{E}+23$ & $2.64 \mathrm{E}+23$ \\
\hline 2013 & $9.03 E+22$ & $2.31 \mathrm{E}+23$ & $3.72 \mathrm{E}+23$ & $6.52 \mathrm{E}+22$ & $1.67 \mathrm{E}+23$ & $2.68 \mathrm{E}+23$ \\
\hline 2014 & $9.02 \mathrm{E}+22$ & $2.31 \mathrm{E}+23$ & $3.71 \mathrm{E}+23$ & $6.66 \mathrm{E}+22$ & $1.70 \mathrm{E}+23$ & $2.74 \mathrm{E}+23$ \\
\hline 2015 & $9.04 \mathrm{E}+22$ & $2.31 \mathrm{E}+23$ & $3.72 \mathrm{E}+23$ & $6.76 \mathrm{E}+22$ & $1.73 \mathrm{E}+23$ & $2.78 \mathrm{E}+23$ \\
\hline 2016 & $9.02 \mathrm{E}+22$ & $2.31 \mathrm{E}+23$ & $3.71 \mathrm{E}+23$ & $6.90 \mathrm{E}+22$ & $1.76 \mathrm{E}+23$ & $2.84 \mathrm{E}+23$ \\
\hline 2017 & & & & $6.99 \mathrm{E}+22$ & $1.79 \mathrm{E}+23$ & $2.88 \mathrm{E}+23$ \\
\hline 2018 & & & & $7.13 \mathrm{E}+22$ & $1.82 \mathrm{E}+23$ & $2.93 \mathrm{E}+23$ \\
\hline 2019 & & & & $7.22 \mathrm{E}+22$ & $1.85 \mathrm{E}+23$ & $2.97 \mathrm{E}+23$ \\
\hline 2020 & & & & $7.35 \mathrm{E}+22$ & $1.88 \mathrm{E}+23$ & $3.02 \mathrm{E}+23$ \\
\hline 2021 & & & & $7.44 \mathrm{E}+22$ & $1.90 \mathrm{E}+23$ & $3.06 \mathrm{E}+23$ \\
\hline 2022 & & & & $7.56 \mathrm{E}+22$ & $1.93 \mathrm{E}+23$ & $3.11 \mathrm{E}+23$ \\
\hline 2023 & & & & $7.64 \mathrm{E}+22$ & $1.95 \mathrm{E}+23$ & $3.15 E+23$ \\
\hline
\end{tabular}




\section{Continued}

\begin{tabular}{|c|c|c|c|}
\hline 2024 & $7.76 \mathrm{E}+22$ & $1.98 \mathrm{E}+23$ & $3.19 \mathrm{E}+23$ \\
\hline 2025 & $7.84 \mathrm{E}+22$ & $2.00 \mathrm{E}+23$ & $3.23 \mathrm{E}+23$ \\
\hline 2026 & $7.95 \mathrm{E}+22$ & $2.03 \mathrm{E}+23$ & $3.27 \mathrm{E}+23$ \\
\hline 2027 & $8.02 \mathrm{E}+22$ & $2.05 E+23$ & $3.30 \mathrm{E}+23$ \\
\hline 2028 & $8.12 \mathrm{E}+22$ & $2.08 \mathrm{E}+23$ & $3.34 \mathrm{E}+23$ \\
\hline 2029 & $8.18 \mathrm{E}+22$ & $2.09 \mathrm{E}+23$ & $3.37 \mathrm{E}+23$ \\
\hline 2030 & $8.28 \mathrm{E}+22$ & $2.12 \mathrm{E}+23$ & $3.41 \mathrm{E}+23$ \\
\hline 2031 & $8.33 E+22$ & $2.13 \mathrm{E}+23$ & $3.43 \mathrm{E}+23$ \\
\hline 2032 & $8.42 \mathrm{E}+22$ & $2.15 \mathrm{E}+23$ & $3.47 \mathrm{E}+23$ \\
\hline 2033 & $8.47 \mathrm{E}+22$ & $2.17 \mathrm{E}+23$ & $3.49 \mathrm{E}+23$ \\
\hline 2034 & $8.55 \mathrm{E}+22$ & $2.19 \mathrm{E}+23$ & $3.52 \mathrm{E}+23$ \\
\hline 2035 & $8.59 \mathrm{E}+22$ & $2.20 \mathrm{E}+23$ & $3.54 \mathrm{E}+23$ \\
\hline 2036 & $8.66 \mathrm{E}+22$ & $2.21 \mathrm{E}+23$ & $3.57 \mathrm{E}+23$ \\
\hline 2037 & $8.69 \mathrm{E}+22$ & $2.22 \mathrm{E}+23$ & $3.58 \mathrm{E}+23$ \\
\hline 2038 & $8.76 \mathrm{E}+22$ & $2.24 \mathrm{E}+23$ & $3.61 \mathrm{E}+23$ \\
\hline 2039 & $8.79 \mathrm{E}+22$ & $2.25 \mathrm{E}+23$ & $3.62 \mathrm{E}+23$ \\
\hline 2040 & $8.85 \mathrm{E}+22$ & $2.26 \mathrm{E}+23$ & $3.64 \mathrm{E}+23$ \\
\hline 2041 & $8.86 \mathrm{E}+22$ & $2.27 \mathrm{E}+23$ & $3.65 \mathrm{E}+23$ \\
\hline 2042 & $8.91 \mathrm{E}+22$ & $2.28 \mathrm{E}+23$ & $3.67 \mathrm{E}+23$ \\
\hline 2043 & $8.93 \mathrm{E}+22$ & $2.28 \mathrm{E}+23$ & $3.67 \mathrm{E}+23$ \\
\hline 2044 & $8.97 \mathrm{E}+22$ & $2.29 \mathrm{E}+23$ & $3.69 \mathrm{E}+23$ \\
\hline 2045 & $8.97 \mathrm{E}+22$ & $2.29 \mathrm{E}+23$ & $3.69 \mathrm{E}+23$ \\
\hline 2046 & $9.01 \mathrm{E}+22$ & $2.30 \mathrm{E}+23$ & $3.71 \mathrm{E}+23$ \\
\hline 2047 & $9.00 \mathrm{E}+22$ & $2.30 \mathrm{E}+23$ & $3.71 \mathrm{E}+23$ \\
\hline 2048 & $9.03 \mathrm{E}+22$ & $2.31 \mathrm{E}+23$ & $3.72 \mathrm{E}+23$ \\
\hline 2049 & $9.02 \mathrm{E}+22$ & $2.31 \mathrm{E}+23$ & $3.71 \mathrm{E}+23$ \\
\hline 2050 & $9.04 \mathrm{E}+22$ & $2.31 \mathrm{E}+23$ & $3.72 \mathrm{E}+23$ \\
\hline 2051 & $9.02 \mathrm{E}+22$ & $2.31 \mathrm{E}+23$ & $3.71 \mathrm{E}+23$ \\
\hline
\end{tabular}

EARTH ALBEDO

\begin{tabular}{cccccc}
\cline { 2 - 5 } Year & $\begin{array}{c}\text { (b) Albedo-15\% lower with } \\
\text { offsetor } 2.10 \mathrm{~W} \cdot \mathrm{m}^{-2} .55 \%: 45 \% \\
\text { Grassland: Forest to cropland }\end{array}$ & $\begin{array}{c}\text { (b) Albedo-15\% lower with } \\
\text { offset } 2.10 \mathrm{~W} \cdot \mathrm{m}^{-2} .55 \%: 45 \% \\
\text { Grassland: Forest to cropland }\end{array}$ & temp k $\begin{array}{c}\text { TOA } \\
\text { albedo }\end{array}$ Temp K & $\begin{array}{c}\text { Observed } \\
\text { temp (K) }\end{array}$ \\
\hline 1880 & & & & \\
1881 & & & & \\
1882 & & & \\
1883 & & & \\
1884 & &
\end{tabular}




\section{Continued}

1886

1887

1888

1889

1890

1891

1892

1893

1894

1895

1896

1897

1898

1899

1900

1901

1902

1903

1904

1905

1906

1907

1908

1909

$1910 \quad 0.316065337$

0.316065337

Temp K

$1911 \quad 0.316052906$

0.316052906

$\begin{array}{llllll}253.16 & 0.3161 & 253.16 & 0.3161 & 286.16 & 286.16\end{array}$

1912

0.316040712

1913

0.316016323

0.316040712

253.16

0.3161

253.16

0.3161

286.16

286.16

253.16

$\begin{array}{lllll}0.3160 & 253.16 & 0.3160 & 286.16 & 286.16\end{array}$

0.316016323

253.17

$\begin{array}{lllll}0.3160 & 253.17 & 0.3160 & 286.17 & 286.17\end{array}$

1914

0.315992407

0.315992407

253.17

0.3160

253.17

$0.3160 \quad 286.17$

286.17

0.315956531

253.17

0.3160

$253.17 \quad 0.3160 \quad 286.1$

286.17

0.315921365

253.18

0.3159

253.18

$0.3159 \quad 286.18$

286.18

0.315874474

253.18

0.3159

253.1

$\begin{array}{lll}0.3159 & 286.18 \quad 286.18\end{array}$

0.315828528

253.18

0.3158

253.18

$286.18 \quad 286.18$

0.315771092

253.19

$\begin{array}{lllll}0.3158 & 253.19 & 0.3158 & 286.19 & 286.19\end{array}$

0.315714837

253.19

0.3157

$286.19 \quad 286.19$

0.315639682

253.20

0.3156

253.2

0.3156

$286.20 \quad 286.20$

0.315566103

$\begin{array}{llllll}253.21 & 0.3156 & 253.21 & 0.3156 & 286.21 & 286.21\end{array}$

1922

0.315566103

0.315474016

$253.22 \quad 0.3155$

$253.22 \quad 0.3155 \quad 286.22 \quad 286.22$ 


\section{Continued}

\begin{tabular}{|c|c|c|c|c|c|c|c|c|}
\hline 1924 & 0.315383901 & 0.315383901 & 253.22 & 0.3154 & 253.22 & 0.3154 & 286.22 & 286.22 \\
\hline 1925 & 0.315275668 & 0.315275668 & 253.23 & 0.3153 & 253.23 & 0.3153 & 286.23 & 286.23 \\
\hline 1926 & 0.315169801 & 0.315169801 & 253.24 & 0.3152 & 253.24 & 0.3152 & 286.24 & 286.24 \\
\hline 1927 & 0.315046208 & 0.315046208 & 253.26 & 0.3150 & 253.26 & 0.3150 & 286.26 & 286.26 \\
\hline 1928 & 0.314925375 & 0.314925375 & 253.27 & 0.3149 & 253.27 & 0.3149 & 286.27 & 286.27 \\
\hline 1929 & 0.314787206 & 0.314787206 & 253.28 & 0.3148 & 253.28 & 0.3148 & 286.28 & 286.28 \\
\hline 1930 & 0.314652192 & 0.314652192 & 253.29 & 0.3147 & 253.29 & 0.3147 & 286.29 & 286.29 \\
\hline 1931 & 0.314499006 & 0.314499006 & 253.31 & 0.3145 & 253.31 & 0.3145 & 286.31 & 286.31 \\
\hline 1932 & 0.3143494 & 0.3143494 & 253.32 & 0.3143 & 253.32 & 0.3143 & 286.32 & 286.32 \\
\hline 1933 & 0.314182038 & 0.314182038 & 253.34 & 0.3142 & 253.34 & 0.3142 & 286.34 & 286.34 \\
\hline 1934 & 0.314018679 & 0.314018679 & 253.35 & 0.3140 & 253.35 & 0.3140 & 286.35 & 286.35 \\
\hline 1935 & 0.313837981 & 0.313837981 & 253.37 & 0.3138 & 253.37 & 0.3138 & 286.37 & 286.37 \\
\hline 1936 & 0.31366171 & 0.31366171 & 253.38 & 0.3137 & 253.38 & 0.3137 & 286.38 & 286.38 \\
\hline 1937 & 0.313468515 & 0.313468515 & 253.40 & 0.3135 & 253.40 & 0.3135 & 286.40 & 286.40 \\
\hline 1938 & 0.313280172 & 0.313280172 & 253.42 & 0.3133 & 253.42 & 0.3133 & 286.42 & 286.42 \\
\hline 1939 & 0.313075321 & 0.313075321 & 253.44 & 0.3131 & 253.44 & 0.3131 & 286.44 & 286.44 \\
\hline 1940 & 0.312875747 & 0.312875747 & 253.46 & 0.3129 & 253.46 & 0.3129 & 286.46 & 286.46 \\
\hline 1941 & 0.312662756 & 0.312662756 & 253.48 & 0.3127 & 253.48 & 0.3127 & 286.48 & 286.48 \\
\hline 1942 & 0.312455398 & 0.312455398 & 253.50 & 0.3125 & 253.50 & 0.3125 & 286.50 & 286.50 \\
\hline 1943 & 0.31223497 & 0.31223497 & 253.52 & 0.3122 & 253.52 & 0.3122 & 286.52 & 286.52 \\
\hline 1944 & 0.31202053 & 0.31202053 & 253.54 & 0.3120 & 253.54 & 0.3120 & 286.54 & 286.54 \\
\hline 1945 & 0.311793368 & 0.311793368 & 253.56 & 0.3118 & 253.56 & 0.3118 & 286.56 & 286.56 \\
\hline 1946 & 0.311572552 & 0.311572552 & 253.58 & 0.3116 & 253.58 & 0.3116 & 286.58 & 286.58 \\
\hline 1947 & 0.311339361 & & 253.60 & 0.3113 & & & & 286.60 \\
\hline 1948 & 0.311112873 & & 253.62 & 0.3111 & & & & 286.62 \\
\hline 1949 & 0.310874359 & & 253.64 & 0.3109 & & & & 286.64 \\
\hline 1950 & 0.310642905 & & 253.66 & 0.3106 & & & & 286.66 \\
\hline 1951 & 0.310399775 & & 253.68 & 0.3104 & & & & 286.68 \\
\hline 1952 & 0.310164064 & & 253.71 & 0.3102 & & & & 286.71 \\
\hline 1953 & 0.309917026 & & 253.73 & 0.3099 & & & & 286.73 \\
\hline 1954 & 0.309677767 & & 253.75 & 0.3097 & & & & 286.75 \\
\hline 1955 & 0.309427531 & & 253.77 & 0.3094 & & & & 286.77 \\
\hline 1956 & 0.309185435 & & 253.80 & 0.3092 & & & & 286.80 \\
\hline 1957 & 0.308932711 & & 253.82 & 0.3089 & & & & 286.82 \\
\hline 1958 & 0.308688489 & & 253.84 & 0.3087 & & & & 286.84 \\
\hline 1959 & 0.308433992 & & 253.87 & 0.3084 & & & & 286.87 \\
\hline 1960 & 0.308188358 & & 253.89 & 0.3082 & & & & 286.89 \\
\hline 1961 & 0.307932801 & & 253.91 & 0.3079 & & & & 286.91 \\
\hline
\end{tabular}




\section{Continued}

\begin{tabular}{|c|c|c|c|c|c|c|c|c|}
\hline 1962 & 0.30768647 & & 253.93 & 0.3077 & & & & 286.93 \\
\hline 1963 & 0.30743057 & & 253.96 & 0.3074 & & & & 286.96 \\
\hline 1964 & 0.307184258 & & 253.98 & 0.3072 & & & & 286.98 \\
\hline 1965 & 0.306928731 & & 254.00 & 0.3069 & & & & 287.00 \\
\hline 1966 & 0.306683158 & & 254.03 & 0.3067 & & & & 287.03 \\
\hline 1967 & 0.306428723 & & 254.05 & 0.3064 & & & & 287.05 \\
\hline 1968 & 0.306184607 & & 254.07 & 0.3062 & & & & 287.07 \\
\hline 1969 & 0.305931985 & & 254.09 & 0.3059 & & & & 287.09 \\
\hline 1970 & 0.305690048 & & 254.12 & 0.3057 & & & & 287.12 \\
\hline 1971 & 0.30538363 & & 254.14 & 0.3054 & & & & 287.14 \\
\hline 1972 & 0.305150437 & & 254.17 & 0.3052 & & & & 287.17 \\
\hline 1973 & 0.304852881 & & 254.19 & 0.3049 & & & & 287.19 \\
\hline 1974 & 0.304628748 & & 254.21 & 0.3046 & & & & 287.21 \\
\hline 1975 & 0.304340371 & & 254.24 & 0.3043 & & & & 287.24 \\
\hline 1976 & 0.304125612 & & 254.26 & 0.3041 & & & & 287.26 \\
\hline 1977 & 0.303846731 & & 254.28 & 0.3038 & & & & 287.28 \\
\hline 1978 & 0.303641662 & & 254.30 & 0.3036 & & & & 287.30 \\
\hline 1979 & 0.303372594 & & 254.33 & 0.3034 & & & & 287.33 \\
\hline 1980 & 0.303177531 & & 254.35 & 0.3032 & & & & 287.35 \\
\hline 1981 & 0.302915701 & 0.311572552 & 254.37 & 0.3029 & 253.58 & 0.3116 & 286.58 & 287.37 \\
\hline 1982 & 0.302728232 & 0.311339361 & 254.39 & 0.3027 & 253.60 & 0.3113 & 286.60 & 287.39 \\
\hline 1983 & 0.302474283 & 0.311112873 & 254.41 & 0.3025 & 253.62 & 0.3111 & 286.62 & 287.41 \\
\hline 1984 & 0.302295052 & 0.310874359 & 254.43 & 0.3023 & 253.64 & 0.3109 & 286.64 & 287.43 \\
\hline 1985 & 0.302049628 & 0.310642905 & 254.45 & 0.3020 & 253.66 & 0.3106 & 286.66 & 287.45 \\
\hline 1986 & 0.301879277 & 0.310399775 & 254.46 & 0.3019 & 253.68 & 0.3104 & 286.68 & 287.46 \\
\hline 1987 & 0.301643024 & 0.310164064 & 254.49 & 0.3016 & 253.71 & 0.3102 & 286.71 & 287.49 \\
\hline 1988 & 0.301482196 & 0.309917026 & 254.50 & 0.3015 & 253.73 & 0.3099 & 286.73 & 287.50 \\
\hline 1989 & 0.301255759 & 0.309677767 & 254.52 & 0.3013 & 253.75 & 0.3097 & 286.75 & 287.52 \\
\hline 1990 & 0.301105099 & 0.309427531 & 254.53 & 0.3011 & 253.77 & 0.3094 & 286.77 & 287.53 \\
\hline 1991 & 0.300893544 & 0.309185435 & 254.55 & 0.3009 & 253.80 & 0.3092 & 286.80 & 287.55 \\
\hline 1992 & 0.300757763 & 0.308932711 & 254.57 & 0.3008 & 253.82 & 0.3089 & 286.82 & 287.57 \\
\hline 1993 & 0.300561033 & 0.308688489 & 254.58 & 0.3006 & 253.84 & 0.3087 & 286.84 & 287.58 \\
\hline 1994 & 0.300440073 & 0.308433992 & 254.60 & 0.3004 & 253.87 & 0.3084 & 286.87 & 287.60 \\
\hline 1995 & 0.30025811 & 0.308188358 & 254.61 & 0.3003 & 253.89 & 0.3082 & 286.89 & 287.61 \\
\hline 1996 & 0.300151911 & 0.307932801 & 254.62 & 0.3002 & 253.91 & 0.3079 & 286.91 & 287.62 \\
\hline 1997 & 0.299984657 & 0.30768647 & 254.64 & 0.3000 & 253.93 & 0.3077 & 286.93 & 287.64 \\
\hline 1998 & 0.299893156 & 0.30743057 & 254.65 & 0.2999 & 253.96 & 0.3074 & 286.96 & 287.65 \\
\hline 1999 & 0.29974055 & 0.307184258 & 254.66 & 0.2997 & 253.98 & 0.3072 & 286.98 & 287.66 \\
\hline
\end{tabular}


Continued

\begin{tabular}{|c|c|c|c|c|c|c|c|c|}
\hline 2000 & 0.299663684 & 0.306928731 & 254.67 & 0.2997 & 254.00 & 0.3069 & 287.00 & 287.67 \\
\hline 2001 & 0.299522065 & 0.306683158 & 254.68 & 0.2995 & 254.03 & 0.3067 & 287.03 & 287.68 \\
\hline 2002 & 0.299460133 & 0.306428723 & 254.68 & 0.2995 & 254.05 & 0.3064 & 287.05 & 287.68 \\
\hline 2003 & 0.299330941 & 0.306184607 & 254.69 & 0.2993 & 254.07 & 0.3062 & 287.07 & 287.69 \\
\hline 2004 & 0.299280124 & 0.305931985 & 254.70 & 0.2993 & 254.09 & 0.3059 & 287.09 & 287.70 \\
\hline 2005 & 0.29916625 & 0.305690048 & 254.71 & 0.2992 & 254.12 & 0.3057 & 287.12 & 287.71 \\
\hline 2006 & 0.299130571 & 0.30538363 & 254.72 & 0.2991 & 254.14 & 0.3054 & 287.14 & 287.72 \\
\hline 2007 & 0.299033475 & 0.305150437 & 254.72 & 0.2990 & 254.17 & 0.3052 & 287.17 & 287.72 \\
\hline 2008 & 0.299011136 & 0.304852881 & 254.73 & 0.2990 & 254.19 & 0.3049 & 287.19 & 287.73 \\
\hline 2009 & 0.298927611 & 0.304628748 & 254.73 & 0.2989 & 254.21 & 0.3046 & 287.21 & 287.73 \\
\hline 2010 & 0.298921702 & 0.304340371 & 254.74 & 0.2989 & 254.24 & 0.3043 & 287.24 & 287.74 \\
\hline 2011 & 0.298851239 & 0.304125612 & 254.74 & 0.2989 & 254.26 & 0.3041 & 287.26 & 287.74 \\
\hline 2012 & 0.298858973 & 0.303846731 & 254.75 & 0.2989 & 254.28 & 0.3038 & 287.28 & 287.75 \\
\hline 2013 & 0.298804407 & 0.303641662 & 254.74 & 0.2988 & 254.30 & 0.3036 & 287.30 & 287.74 \\
\hline 2014 & 0.298826979 & 0.303372594 & 254.75 & 0.2988 & 254.33 & 0.3034 & 287.33 & 287.75 \\
\hline 2015 & 0.298788269 & 0.303177531 & 254.75 & 0.2988 & 254.35 & 0.3032 & 287.35 & 287.75 \\
\hline 2016 & 0.298826979 & 0.302915701 & 254.75 & 0.2988 & 254.37 & 0.3029 & 287.37 & 287.75 \\
\hline 2017 & & 0.302728232 & & & 254.39 & 0.3027 & 287.39 & \\
\hline 2018 & & 0.302474283 & & & 254.41 & 0.3025 & 287.41 & \\
\hline 2019 & & 0.302295052 & & & 254.43 & 0.3023 & 287.43 & \\
\hline 2020 & & 0.302049628 & & & 254.45 & 0.3020 & 287.45 & \\
\hline 2021 & & 0.301879277 & & & 254.46 & 0.3019 & 287.46 & \\
\hline 2022 & & 0.301643024 & & & 254.49 & 0.3016 & 287.49 & \\
\hline 2023 & & 0.301482196 & & & 254.50 & 0.3015 & 287.50 & \\
\hline 2024 & & 0.301255759 & & & 254.52 & 0.3013 & 287.52 & \\
\hline 2025 & & 0.301105099 & & & 254.53 & 0.3011 & 287.53 & \\
\hline 2026 & & 0.300893544 & & & 254.55 & 0.3009 & 287.55 & \\
\hline 2027 & & 0.300757763 & & & 254.57 & 0.3008 & 287.57 & \\
\hline 2028 & & 0.300561033 & & & 254.58 & 0.3006 & 287.58 & \\
\hline 2029 & & 0.300440073 & & & 254.60 & 0.3004 & 287.60 & \\
\hline 2030 & & 0.30025811 & & & 254.61 & 0.3003 & 287.61 & \\
\hline 2031 & & 0.300151911 & & & 254.62 & 0.3002 & 287.62 & \\
\hline 2032 & & 0.299984657 & & & 254.64 & 0.3000 & 287.64 & \\
\hline 2033 & & 0.299893156 & & & 254.65 & 0.2999 & 287.65 & \\
\hline 2034 & & 0.29974055 & & & 254.66 & 0.2997 & 287.66 & \\
\hline 2035 & & 0.299663684 & & & 254.67 & 0.2997 & 287.67 & \\
\hline 2036 & & 0.299522065 & & & 254.68 & 0.2995 & 287.68 & \\
\hline 2037 & & 0.299460133 & & & 254.68 & 0.2995 & 287.68 & \\
\hline
\end{tabular}




\begin{tabular}{llllll} 
Continued & & & & \\
\hline 2038 & 0.299330941 & 254.69 & 0.2993 & 287.69 \\
2039 & 0.299280124 & 254.70 & 0.2993 & 287.70 \\
2040 & 0.29916625 & 254.71 & 0.2992 & 287.71 \\
2041 & 0.299130571 & 254.72 & 0.2991 & 287.72 \\
2042 & 0.299033475 & 254.72 & 0.2990 & 287.72 \\
2043 & 0.299011136 & 254.73 & 0.2990 & 287.73 \\
2044 & 0.298927611 & 254.73 & 0.2989 & 287.73 \\
2045 & 0.298921702 & 254.74 & 0.2989 & 287.74 \\
2046 & 0.298851239 & 254.74 & 0.2989 & 287.74 \\
2047 & 0.298858973 & 254.75 & 0.2989 & 287.75 \\
2048 & 0.298804407 & 254.74 & 0.2988 & 287.74 \\
2049 & 0.298826979 & 254.75 & 0.2988 & 287.75 \\
2050 & 0.298788269 & 254.75 & 0.2988 & 287.75 \\
2051 & 0.298826979 & 254.75 & 0.2988 & 287.75 \\
\hline
\end{tabular}

